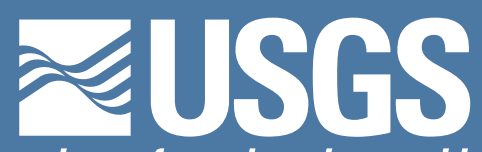

science for a changing world

Prepared in cooperation with the Navajo Nation Environmental Protection Agency

\title{
The Source of Groundwater and Solutes to Many Devils Wash at a Former Uranium Mill Site in Shiprock, New Mexico
}

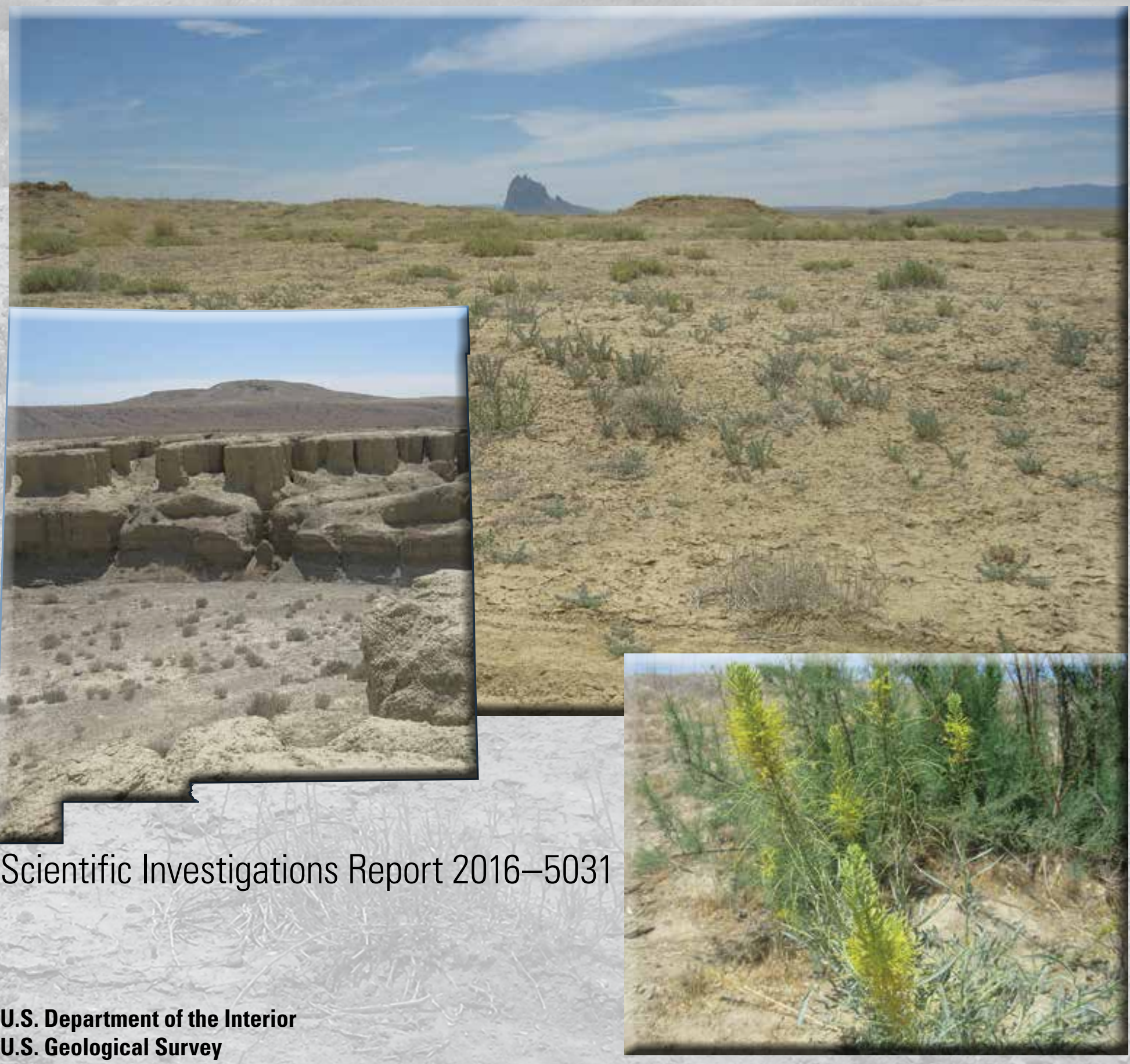


Cover:

Top, Volcanic remnant Shiprock as seen from the Many Devils Wash arroyo near Shiprock, New

Mexico.

Left, Piping features on the east bluff of Many Devils Wash.

Right, Prince's Plume growing near the Shiprock Disposal Site, Shiprock, New Mexico.

All photographs were taken by Andrew Robertson, U.S. Geological Survey. 


\section{The Source of Groundwater and Solutes to Many Devils Wash at a Former Uranium Mill Site in Shiprock, New Mexico}

By Andrew J. Robertson, Anthony J. Ranalli, Stephen A. Austin, and

Bryan R. Lawlis

Prepared in cooperation with the Navajo Nation Environmental Protection Agency

Scientific Investigations Report 2016-5031 


\title{
U.S. Department of the Interior SALLY JEWELL, Secretary
}

\section{U.S. Geological Survey Suzette M. Kimball, Director}

\author{
U.S. Geological Survey, Reston, Virginia: 2016
}

For more information on the USGS - the Federal source for science about the Earth, its natural and living resources, natural hazards, and the environment—visit http://www.usgs.gov or call 1-888-ASK-USGS.

For an overview of USGS information products, including maps, imagery, and publications, visit http://www.usgs.gov/pubprod/.

Any use of trade, firm, or product names is for descriptive purposes only and does not imply endorsement by the U.S. Government.

Although this information product, for the most part, is in the public domain, it also may contain copyrighted materials as noted in the text. Permission to reproduce copyrighted items must be secured from the copyright owner.

Suggested citation:

Robertson, A.J., Ranalli, A.J., Austin, S.A., and Lawlis, B.R., 2016, The source of groundwater and solutes to Many Devils Wash at a former uranium mill site in Shiprock, New Mexico: U.S. Geological Survey Scientific Investigations Report 2016-5031, 54 p., http://dx.doi.org/10.3133/sir20165031.

ISSN 2328-0328 (online) 


\section{Acknowledgments}

The authors would like to acknowledge the people of the Navajo Nation for their support and insight on this investigation. In addition, U.S. Department of Energy personnel provided data, site access, and valuable assistance. 



\section{Contents}

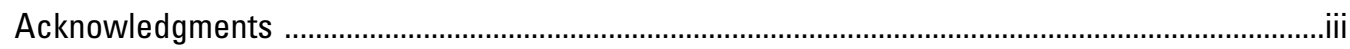

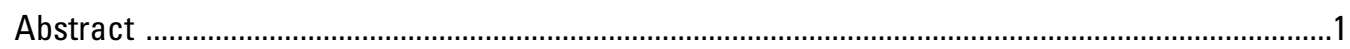



Purpose and Scope ...................................................................................................

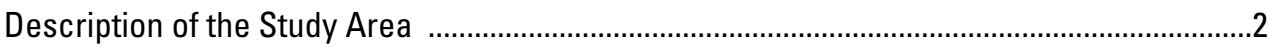



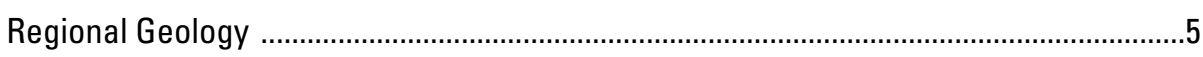

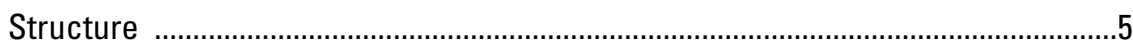

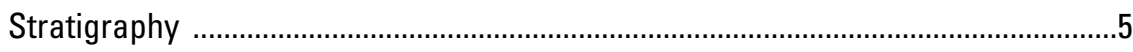

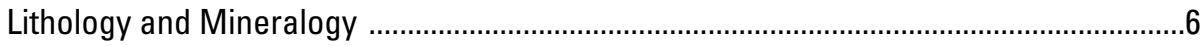

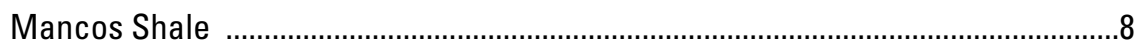

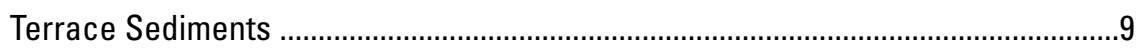

Climate and Vegetation .....................................................................................

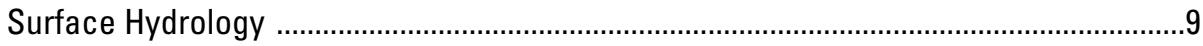

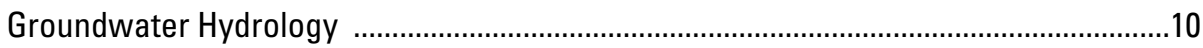

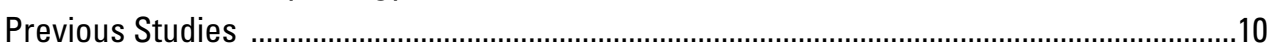

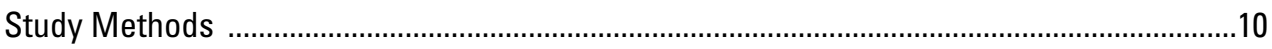

Apparent Groundwater-Age Analysis .........................................................................17

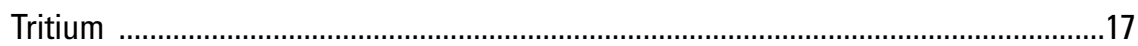

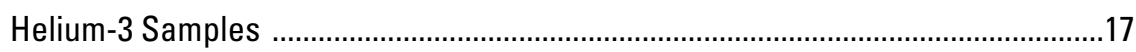

Carbon-13 and Carbon-14 …………......................................................................

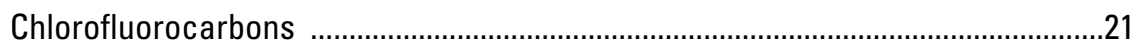

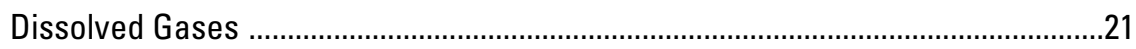

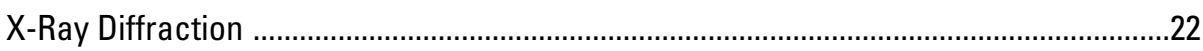

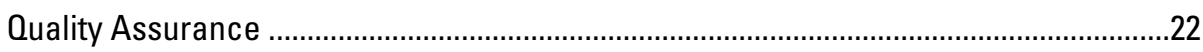





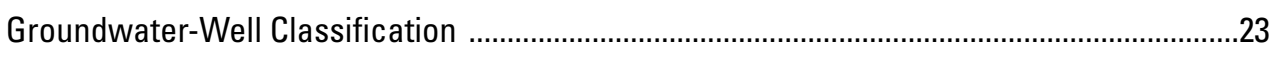

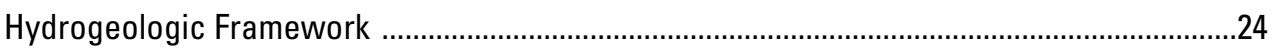

Potential Sources of Recharge to the Area of Many Devils Wash .................................24

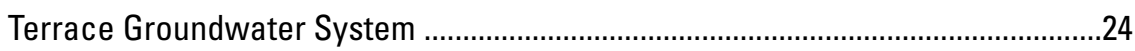

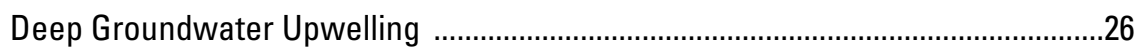



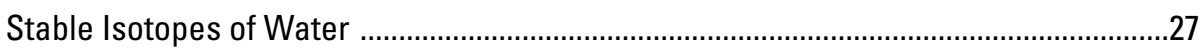

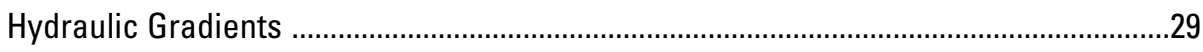

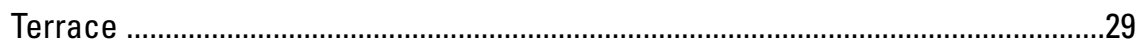

Groundwater Elevations Beneath Many Devils Wash ..........................................31

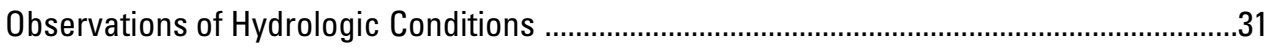

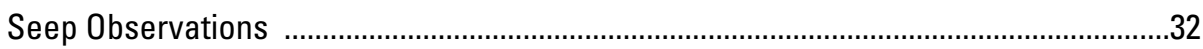

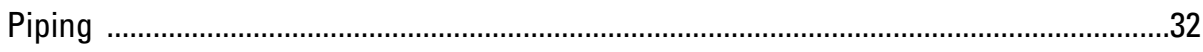

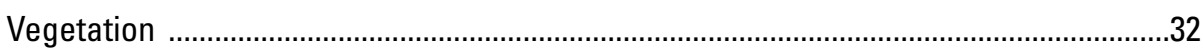




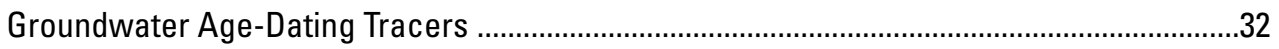

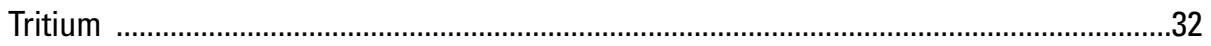

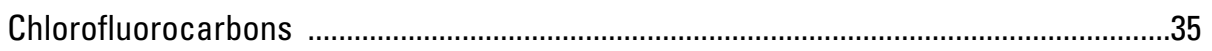

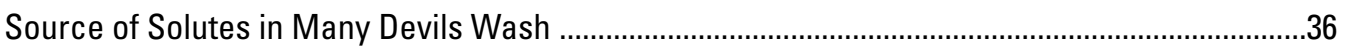

Conceptual Model of Water Quality at the Shiprock Disposal Site............................................36

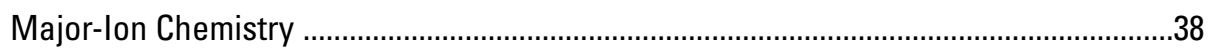

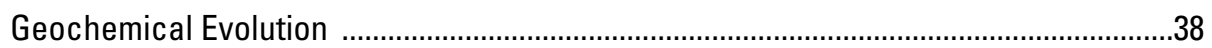

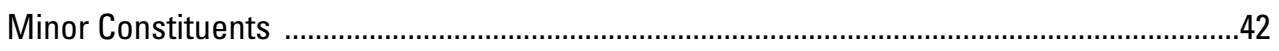

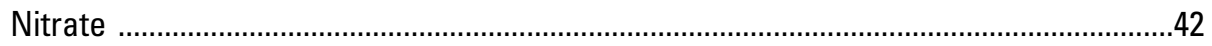

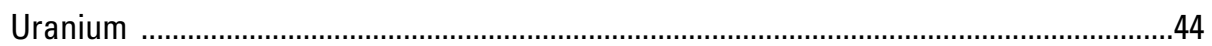

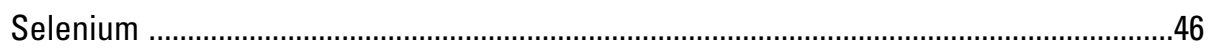

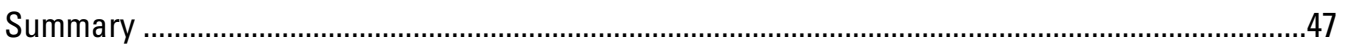

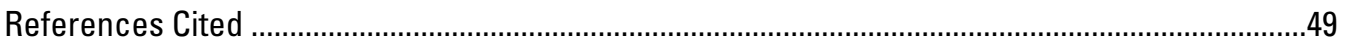

\section{Figures}

1. Map showing the location of Many Devils Wash and analog sites in relation to the Shiprock Disposal Site, Shiprock, New Mexico; inset regional map showing San Juan Basin geologic structures

2. Aerial photograph of the Navajo Mill, Shiprock, New Mexico, during its operation in 1965

3. Stratigraphic column of the San Juan Basin, New Mexico

4. Map image showing well locations and sampling sites at and near the Shiprock Disposal Site, Shiprock, New Mexico, and line of section shown in figure 8

5. Photograph showing efflorescent deposits in Many Devils Wash at the Shiprock Disposal Site, New Mexico, looking upstream (south) at the knickpoint and showing the outcrop of the siltstone bed and terrace gravels

6. Graph showing uranium-234/238 alpha activity ratios and concentrations of uranium in groundwater at and near the Shiprock Disposal Site, Shiprock, New Mexico

7. Graph showing the delta deuterium $(\delta D)$ and delta oxygen-18 $\left(\delta^{18} 0\right)$ composition of selected samples from the study area at and near the Shiprock Disposal Site, Shiprock New Mexico

8. Diagram of well construction relative to lithology and the August 2012 groundwater elevations in the monitoring wells along the east mill flow path between the disposal cell and Many Devils Wash at the Shiprock Disposal Site, New Mexico

9. Graph showing the elevation of groundwater and bedrock surfaces along Many Devils Wash, New Mexico, June 2013

10. Graph showing tritium $\left({ }^{3} \mathrm{H}\right)$ values for groundwater collected from wells at the Shiprock Disposal Site, November 2012, with projected decay curves and precipitation concentrations measured in Albuquerque, New Mexico, 1950-2013 .

11. Graph showing chlorofluorocarbon (CFC) concentrations measured in groundwater from selected wells at the Shiprock Disposal Site, New Mexico, and historical concentrations of CFCs in the North American atmosphere 
12. Piper diagram showing major-ion chemistry of groundwater samples at and near the Shiprock Disposal Site and surface-water samples from the San Juan River, New Mexico

13. Graph showing sodium- and chloride-ion concentrations in select groundwater and surface-water samples at and near the Shiprock Disposal Site, New Mexico . .41

14. Graph showing sodium and nitrate ion concentrations in select Shiprock, New Mexico, groundwater and surface-water samples .44

15. Boxplot showing distribution of uranium (U) concentrations in groundwater samples at and near the Shiprock Disposal Site, Shiprock, New Mexico, regional Mancos Shale seeps reported by the U.S. Department of Energy (2011b), and soil and shale extracts from Mancos Shale reported by Tuttle and others (2007) . .45

16. Boxplot showing distribution of distribution of selenium concentrations in groundwater samples at and near the Shiprock Disposal Site, Shiprock, New Mexico, regional Mancos Shale seeps reported by the U.S. Department of Energy (2011b), and soil and shale extracts from the Mancos Shale reported by Tuttle and others (2007)

\section{Tables}

1. Shiprock Disposal Site well information and selected sample data, Shiprock, New Mexico

2. Field parameters and analytical results from U.S. Geological Survey sampling event in October and November 2012

3. Historical groundwater elevations in monitoring wells $830,603,731,1058$, and 1059 at the Shiprock Disposal Site, New Mexico

4. Mineralogy, in weight percentage, of selected solid samples collected from the Shiprock, New Mexico, area

5. Coefficient of determination for the least squares regression and $p$-values of ions to specific conductivities 


\section{Conversion Factors}

U.S. customary units to International System of Units

\begin{tabular}{|c|c|c|}
\hline Multiply & By & To obtain \\
\hline \multicolumn{3}{|c|}{ Length } \\
\hline inch (in.) & 2.54 & centimeter $(\mathrm{cm})$ \\
\hline inch (in.) & 25.4 & millimeter (mm) \\
\hline foot $(\mathrm{ft})$ & 0.3048 & meter $(\mathrm{m})$ \\
\hline mile (mi) & 1.609 & kilometer $(\mathrm{km})$ \\
\hline \multicolumn{3}{|c|}{ Area } \\
\hline acre & 4,047 & square meter $\left(\mathrm{m}^{2}\right)$ \\
\hline acre & 0.4047 & hectare (ha) \\
\hline acre & 0.4047 & square hectometer $\left(\mathrm{hm}^{2}\right)$ \\
\hline acre & 0.004047 & square kilometer $\left(\mathrm{km}^{2}\right)$ \\
\hline square mile $\left(\mathrm{mi}^{2}\right)$ & 259.0 & hectare (ha) \\
\hline square mile $\left(\mathrm{mi}^{2}\right)$ & 2.590 & square kilometer $\left(\mathrm{km}^{2}\right)$ \\
\hline \multicolumn{3}{|c|}{ Volume } \\
\hline gallon (gal) & $3,785.41$ & milliliter $(\mathrm{mL})$ \\
\hline gallon (gal) & 3.785 & liter (L) \\
\hline gallon (gal) & 0.003785 & cubic meter $\left(\mathrm{m}^{3}\right)$ \\
\hline gallon (gal) & 3.785 & cubic decimeter $\left(\mathrm{dm}^{3}\right)$ \\
\hline million gallons (Mgal) & 3,785 & cubic meter $\left(\mathrm{m}^{3}\right)$ \\
\hline cubic yard $\left(\mathrm{yd}^{3}\right)$ & 0.7646 & cubic meter $\left(\mathrm{m}^{3}\right)$ \\
\hline \multicolumn{3}{|c|}{ Flow rate } \\
\hline cubic foot per second $\left(\mathrm{ft}^{3} / \mathrm{s}\right)$ & 0.02832 & cubic meter per second $\left(\mathrm{m}^{3} / \mathrm{s}\right)$ \\
\hline gallon per minute (gal/min) & 0.06309 & liter per second $(\mathrm{L} / \mathrm{s})$ \\
\hline inch per year (in/yr) & 25.4 & millimeter per year $(\mathrm{mm} / \mathrm{yr})$ \\
\hline \multicolumn{3}{|c|}{ Mass } \\
\hline ton, short $(2,000 \mathrm{lb})$ & 0.9072 & megagram $(\mathrm{Mg})$ \\
\hline pound, avoirdupois (lb) & 0.4536 & kilogram (kg) \\
\hline \multicolumn{3}{|c|}{ Radioactivity } \\
\hline picocurie per liter $(\mathrm{pCi} / \mathrm{L})$ & 0.037 & becquerel per liter $(\mathrm{Bq} / \mathrm{L})$ \\
\hline
\end{tabular}

Temperature in degrees Celsius $\left({ }^{\circ} \mathrm{C}\right)$ may be converted to degrees Fahrenheit $\left({ }^{\circ} \mathrm{F}\right)$ as follows: ${ }^{\circ} \mathrm{F}=\left(1.8 \times{ }^{\circ} \mathrm{C}\right)+32$.

Temperature in degrees Fahrenheit $\left({ }^{\circ} \mathrm{F}\right)$ may be converted to degrees Celsius $\left({ }^{\circ} \mathrm{C}\right)$ as follows: ${ }^{\circ} \mathrm{C}=\left({ }^{\circ} \mathrm{F}-32\right) / 1.8$.

\section{Datum}

Vertical coordinate information is referenced to the North American Vertical Datum of 1988 (NAVD 88).

Horizontal coordinate information is referenced to the North American Datum of 1983 (NAD 83). 


\section{Supplemental Information}

Elevation, as used in this report, refers to distance above the vertical datum.

Specific conductance is given in microsiemens per centimeter at 25 degrees Celsius $(\mu \mathrm{S} / \mathrm{cm}$ at $\left.25^{\circ} \mathrm{C}\right)$.

Concentrations of chemical constituents in water are given either in milligrams per liter (mg/L) or micrograms per liter $(\mu \mathrm{g} / \mathrm{L})$.

The term mesh, as used in this report, refers to the number of openings across one inch of the screen. A 100-mesh screen has 100 openings and is equal to 0.0059 inches or 0.149 millimeters.

\section{Abbreviations}

\begin{tabular}{|c|c|}
\hline$>$ & Greater than \\
\hline \pm & Plus or minus \\
\hline$\delta^{13} \mathrm{C}$ & Delta carbon-13 \\
\hline$\delta \mathrm{D}$ & Delta deuterium \\
\hline$\delta^{18} \mathrm{O}$ & Delta oxygen -18 \\
\hline$\delta^{34} \mathrm{~S}_{\text {sulfate }}$ & Delta sulfur-34 in sulfate \\
\hline${ }^{3} \mathrm{H}$ & Tritium \\
\hline${ }^{3} \mathrm{He}$ & Helium-3 \\
\hline${ }^{4} \mathrm{He}$ & Helium-4 \\
\hline${ }^{13} \mathrm{C}$ & Carbon-13 \\
\hline${ }^{14} \mathrm{C}$ & Carbon-14 \\
\hline${ }^{18} \mathrm{O}$ & Oxygen-18 \\
\hline${ }^{234} \mathrm{Th}$ & Thorium - 234 \\
\hline AR & Uranium-234/238 alpha activity ratio \\
\hline $\mathrm{Ar}$ & Argon \\
\hline bls & Below land surface \\
\hline $\mathrm{Ca}$ & Calcium \\
\hline $\mathrm{CFC}$ & Chlorofluorocarbons \\
\hline $\mathrm{CO}_{2}$ & Carbon dioxide \\
\hline $\mathrm{CO}_{3}$ & Carbonate \\
\hline $\mathrm{Cl}$ & Chloride \\
\hline EPA & U.S. Environmental Protection Agency \\
\hline $\mathrm{D}$ & Deuterium \\
\hline $\mathrm{DO}$ & Dissolved oxygen \\
\hline DOE & U.S. Department of Energy \\
\hline $\mathrm{FeOOH}$ & Iron hydroxide \\
\hline $\mathrm{FeS}_{2}$ & Iron sulfide \\
\hline GEMS & Geospatial Environmental Mapping System \\
\hline GMWL & Global Meteoric Water Line \\
\hline $\mathrm{H}^{+}$ & Hydrogen ion \\
\hline
\end{tabular}




\begin{tabular}{|c|c|}
\hline $\mathrm{H}_{2} \mathrm{O}$ & Water \\
\hline $\mathrm{H}_{2} \mathrm{~S}$ & Hydrogen sulfide \\
\hline $\mathrm{HCO}_{3}$ & Bicarbonate \\
\hline $\mathrm{K}$ & Potassium \\
\hline LCS & Laboratory control sample \\
\hline LCSD & Laboratory control sample duplicate \\
\hline LMWL & Local Meteoric Water Line \\
\hline MB & Method blank \\
\hline MCL & Maximum contaminant level \\
\hline $\mathrm{Mg}$ & Magnesium \\
\hline Mill & Navajo Mill \\
\hline MS & Matrix spike \\
\hline MSD & Matrix spike duplicate \\
\hline $\mathrm{N}_{2}$ & Nitrogen \\
\hline $\mathrm{Na}$ & Sodium \\
\hline NECA & Navajo Engineering and Construction Authority \\
\hline $\mathrm{NH}_{3}$ & Ammonia \\
\hline $\mathrm{NO}_{3}$ & Nitrate \\
\hline $\mathrm{O}_{2}$ & Oxygen \\
\hline ppm & Parts per million \\
\hline RASA & Regional Aquifer-System Analysis \\
\hline RPD & Relative percent difference \\
\hline $\mathrm{SC}$ & Specific conductivity \\
\hline $\mathrm{Se}$ & Selenium \\
\hline $\mathrm{SO}_{4}$ & Sulfate \\
\hline TU & Tritium unit \\
\hline UMTRCA & Uranium Mill Tailings Radiation Control Act \\
\hline $\mathrm{U}$ & Uranium \\
\hline $\mathrm{U}_{3} \mathrm{O}_{8}$ & Triuranium octoxide \\
\hline $\mathrm{V}_{2} \mathrm{O}_{5}$ & Vanadium pentoxide \\
\hline
\end{tabular}




\title{
The Source of Groundwater and Solutes to Many Devils Wash at a Former Uranium Mill Site in Shiprock, New Mexico
}

\author{
By Andrew J. Robertson, Anthony J. Ranalli, Stephen A. Austin, and Bryan R. Lawlis
}

\section{Abstract}

The Shiprock Disposal Site is the location of the former Navajo Mill (Mill), a uranium ore-processing facility, located on a terrace overlooking the San Juan River in the town of Shiprock, New Mexico. Following the closure of the Mill, all tailings and associated materials were encapsulated in a disposal cell built on top of the former Mill and tailings piles. The milling operations, conducted at the site from 1954 to 1968 , created radioactive tailings and process-related wastes that are now found in the groundwater. Elevated concentrations of constituents of concern-ammonium, manganese, nitrate, selenium, strontium, sulfate, and uranium - have also been measured in groundwater seeps in the nearby Many Devils Wash arroyo, leading to the inference that these constituents originated from the Mill. These constituents have also been reported in groundwater that is associated with Mancos Shale, the bedrock that underlies the site. The objective of this report is to increase understanding of the source of water and solutes to the groundwater beneath Many Devils Wash and to establish the background concentrations for groundwater that is in contact with the Mancos Shale at the site. This report presents evidence on three working hypotheses: (1) the water and solutes in Many Devils Wash originated from the operations at the former Mill, (2) groundwater in deep aquifers is upwelling under artesian pressure to recharge the shallow groundwater beneath Many Devils Wash, and (3) the groundwater beneath Many Devils Wash originates as precipitation that infiltrates into the shallow aquifer system and discharges to Many Devils Wash in a series of springs on the east side of the wash. The solute concentrations in the shallow groundwater of Many Devils Wash would result from the interaction of the water and the Mancos Shale if the source of water was upwelling from deep aquifers or precipitation.

In order to compare the groundwater from various wells to groundwater that has been affected by Mill activities, a classification system was developed to determine which wells were most likely to have been affected. Affects to groundwater by the Mill were determined by using the reported uranium alpha activity ratios measured in groundwater samples, along with the concentration of the uranium and the location of the wells relative to the Mill. Activity ratios of 1.2 or less were determined to be the most reliable indicator of Millaffected groundwater. Wells with samples that had a reported activity ratio of 1.2 or less were classified as Mill affected. To compare groundwater with background water-quality, data from groundwater seeps and springs in the Upper Eagle Nest Arroyo and Salt Creek Wash, located north of the San Juan River, are also presented and analyzed.

Based on groundwater elevations and tritium concentrations measured in wells located between the disposal cell and Many Devils Wash, Mill water is not likely to reach Many Devils Wash. The tritium concentrations also indicate that groundwater from the Mill has not substantially affected Many Devils Wash in the past. Upwelling from deep aquifers was also determined to be an unlikely source, primarily by comparing the composition of the stable isotopes of water in the shallow groundwater with those reported in groundwater samples from the deeper aquifers. The stable-isotope compositions of the shallow groundwater around the site are enriched relative to the San Juan River and local meteoric lines, which suggests that most of the shallow groundwater has been influenced by evaporation and therefore was recharged at the surface. Several observations indicate that focused recharge is the likely source of groundwater in the area of Many Devils Wash. The visible erosional features in Many Devils Wash provide evidence of piping and groundwater sapping, and the distribution and type of vegetation in Many Devils Wash suggest that the focused recharge of precipitation is occurring. The estimated recharge from precipitation was calculated to be 0.0008 inches per year (in/yr) by using the mass-balance approach from reported seep discharge and $0.0011 \mathrm{in} / \mathrm{yr}$ using the chloride mass-balance approach.

A conceptual model of groundwater quality beneath Many Devils Wash is presented to explain the source of solutes in the groundwater beneath Many Devils Wash. The major-ion concentrations and geochemical evolution in the groundwater beneath Many Devils Wash and across the study area support the conceptual model that the underlying Mancos 
Shale is the source of solutes. Differences in the major-ion composition between groundwater samples collected around the site, result from the degree of weathering to the Mancos Shale. The cation distribution appears to be an indicator of effects from the Mill, with samples from the Millaffected wells largely having a calcium/magnesium-sulfate composition that resembles the reported compositions of more weathered shale; however, that composition could change if the Mill-processed water flowed into areas where the Mancos Shale was less weathered. On the basis of the widespread presence of uranium in the Mancos Shale and the distribution of aqueous uranium in the analog sites and other sites in the region, it appears likely that uranium in the groundwater of Many Devils Wash is naturally sourced from the Mancos Shale.

\section{Introduction}

The Shiprock Disposal Site is a Uranium Mill Tailings Radiation Control Act (UMTRCA) Title 1 site located in northwestern New Mexico in the town of Shiprock, about 30 miles (mi) west of Farmington, N. Mex. (fig. 1). It is the site of a former mill that was located approximately 600 feet $(\mathrm{ft})$ south of the San Juan River (fig. 1) on an elevated graveland loess-covered terrace overlooking the river and its flood plain. The site is managed by the U.S. Department of Energy (DOE) Office of Legacy Management under the Uranium Mill Tailings Remedial Action (UMTRA) project and is currently (2016) undergoing groundwater remediation efforts and site monitoring. The Shiprock Disposal Site is the location of the former Navajo Mill (Mill), a uranium ore-processing facility, which was in operation from 1954 to 1968 (U.S. Department of Energy, 2011c). The site facilities, which included the Mill, ore storage areas, raffinate ponds (ponds that contain spent liquids from the milling process), and tailings piles, occupied about 230 acres leased from the Navajo Nation. The milling operations conducted at the site created radioactive tailings and process-related wastes. Contaminants from the tailings and wastes are now found in the groundwater beneath the terrace and have been transported by the groundwater to seeps and the flood plain of the San Juan River (U.S. Department of Energy, 2000). The constituents of concern are ammonium, manganese, nitrate $\left(\mathrm{NO}_{3}\right)$, selenium $(\mathrm{Se})$, strontium, sulfate $\left(\mathrm{SO}_{4}\right)$, and uranium (U) (U.S. Department of Energy, 2011c). Remediation of the Navajo Mill site was recommended by the U.S. Environmental Protection Agency (EPA) in 1974, and in 1983, the DOE and the Navajo Nation entered into an agreement for site cleanup. By September 1986, all tailings and associated materials (including contaminated materials from offsite properties near the Mill) were encapsulated in a disposal cell built on top of the former Mill and tailings piles. Groundwater monitoring began in the 1980s, and active remediation is currently (2016) taking place (U.S. Department of Energy, 2011c).
To address the need to increase understanding of the source of water and solutes to the groundwater beneath Many Devils Wash, the U.S. Geological Survey (USGS), in cooperation with the Navajo Nation Environmental Protection Agency, designed a study based on three working hypotheses: (1) the water and solutes in Many Devils Wash originated from the operations at the former Mill, (2) groundwater in deep aquifers is upwelling under artesian pressure to recharge the shallow groundwater of Many Devils Wash, and (3) the groundwater beneath Many Devils Wash originates as precipitation that infiltrates into the shallow aquifer system and discharges to Many Devils Wash in a series of springs on the east side of the wash. The solute concentrations in the shallow groundwater of Many Devils Wash would result from the interaction of the water and the Mancos Shale if the source of water was upwelling from deep aquifers or precipitation.

\section{Purpose and Scope}

The purpose of this report is to present evidence for the source of groundwater and solutes discharging to Many Devils Wash and to establish the background concentrations for groundwater that is in contact with the Mancos Shale at the site. The aqueous geochemical, isotopic, and age-dating tracer evidence is evaluated along with the hydrogeologic and mineralogical controls to determine the source of water and solutes in Many Devils Wash. Groundwater samples collected from wells in Many Devils Wash are compared with groundwater samples from seeps and springs in an area that was not affected by the Mill and with groundwater samples from wells known to be affected by the Mill.

\section{Description of the Study Area}

The Shiprock Disposal Site includes the Mill and associated ore storage areas, raffinate ponds, and tailings piles, and occupies about 230 acres, which were leased from the Navajo Nation. A disposal cell containing tailings and associated materials (including contaminated materials from offsite properties near the Mill) is located on top of the former Mill and tailings piles.

\section{The Mill}

The disposal cell is constructed on the location of the former Mill and is located approximately $600 \mathrm{ft}$ south of the San Juan River (fig. 1) on an elevated gravel and loesscovered terrace overlooking the river and its flood plain. A northwest-trending shale cliff, known as the escarpment, is several hundred feet north of the disposal cell and forms the boundary between the flood plain and the nearly flat terrace. 


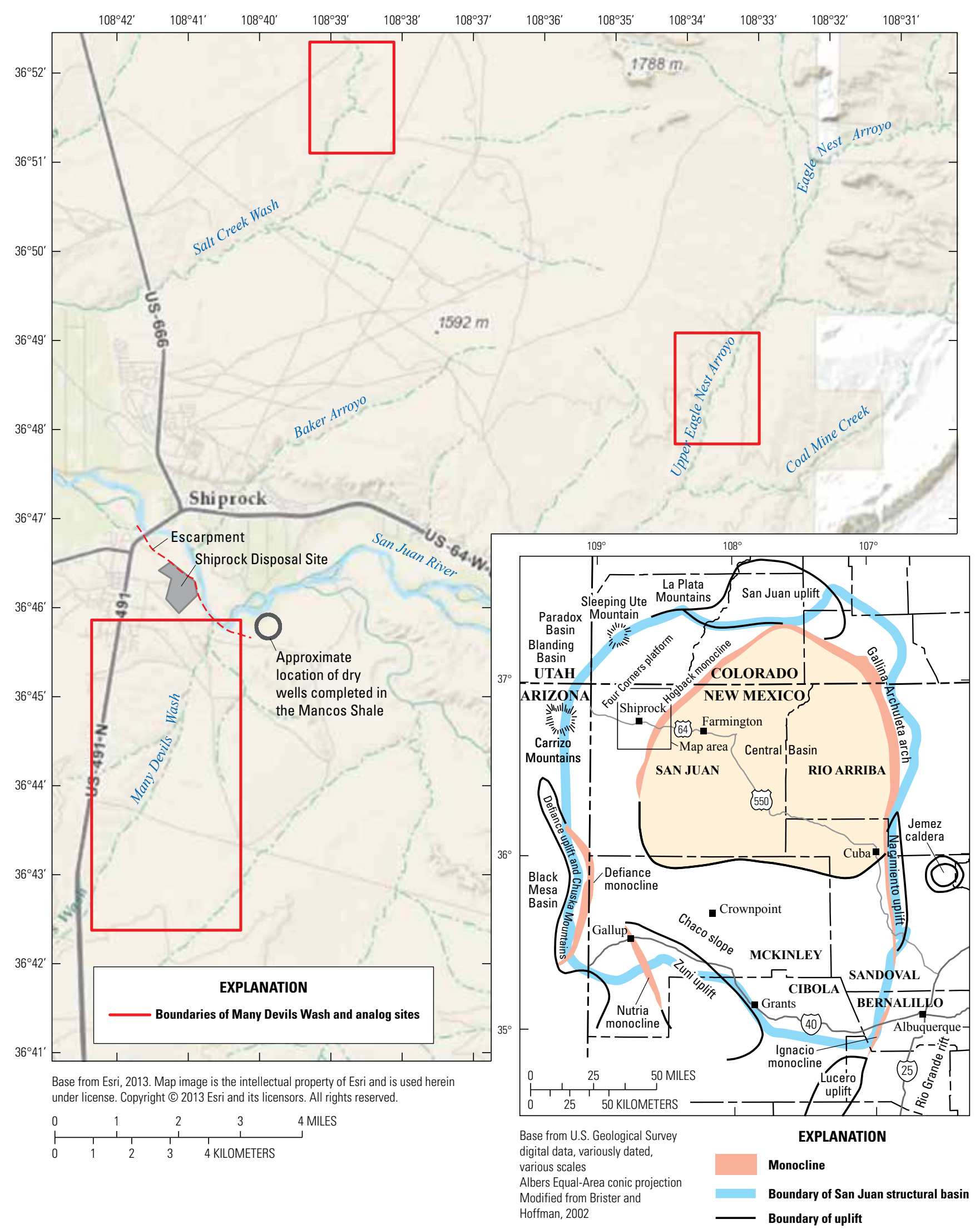

Figure 1. Location of Many Devils Wash and analog sites in relation to the Shiprock Disposal Site, Shiprock, New Mexico; inset regional map showing San Juan Basin geologic structures. 
The Source of Groundwater and Solutes to Many Devils Wash at a Former Uranium Mill Site in Shiprock, New Mexico

The Mill processed about 1.5 million tons of uranium ore from 1954-68 (U.S. Department of Energy, 2011c). The Mill initially processed ore from the Salt Wash Member of the Morrison Formation in the Chuska Mountains of northwestern New Mexico and northeastern Arizona, but the mill later processed ore from various mines in southwestern Colorado, as well as dried slime concentrates and chemical precipitates from regional concentrating plants (Albrethsen and McGinley, 1982). The Mill was able to recover about 94 percent of the uranium and about 58 percent of the vanadium from the ores, resulting in the production of about 7.9 million pounds of triuranium octoxide $\left(\mathrm{U}_{3} \mathrm{O}_{8}\right)$, known as yellow cake, and about 35.4 million pounds of vanadium pentoxide $\left(\mathrm{V}_{2} \mathrm{O}_{5}\right)$ between 1954 and 1968 (U.S. Department of Energy, 2000). The Mill was initially designed to treat low-lime and high-vanadium uranium ores, primarily containing the minerals carnotite and roscoelite, at a capacity of 400 tons per day (Quinn, 1957). The original process (known as the Acid Cure) utilized at the Mill between 1954 and 1957 was developed by the Atomic Energy Commission to increase recovery of vanadium. The Acid Cure process involved blending and curing the ore for more than 6 hours with water and sulfuric acid to convert the uranium and vanadium to soluble salts. Because of a change in the ore feedstock to a high-lime and low-vanadium content and a decline in the vanadium market, the process was changed to the Raw Leach method in 1957 (Quinn, 1957). This method consisted of crushing the ore, leaching with sulfuric acid, and then washing it in classifiers and thickeners. The resulting solution was pumped to a solventextracting circuit where the uranium and vanadium were extracted by using a solution of 5 percent organic solvents (di[2-ethylhexyl] phosphoric acid and tributyl phosphate) and 95 percent kerosene. The uranium was then stripped from the solvent with sodium carbonate, and the carbonate solution was reacidified. The final $\mathrm{U}_{3} \mathrm{O}_{8}$ product was obtained by filtration and drying after it was precipitated by the addition of ammonia $\left(\mathrm{NH}_{3}\right)$ (Public Health Service, 1962).

The two waste streams from the Raw Leach process are the likely source of the process-related constituents in the groundwater underlying the site. The solid material that remained after the ore was crushed and processed was conveyed in a slurry pipeline to two tailings piles located just east of the Mill (fig. 2). The tailings consisted of fine-grained sand, silt, and clay. The raffinate, a term used to describe the liquid waste generated from the final solvent extraction, was piped from the solvent-extraction circuit to evaporation (raffinate) ponds located west and southwest of the tailings ponds (fig. 2). The resulting waste stockpiles of tailings and raffinate ponds were unlined during the entire operational life of the Mill (U.S. Department of Energy, 2000).

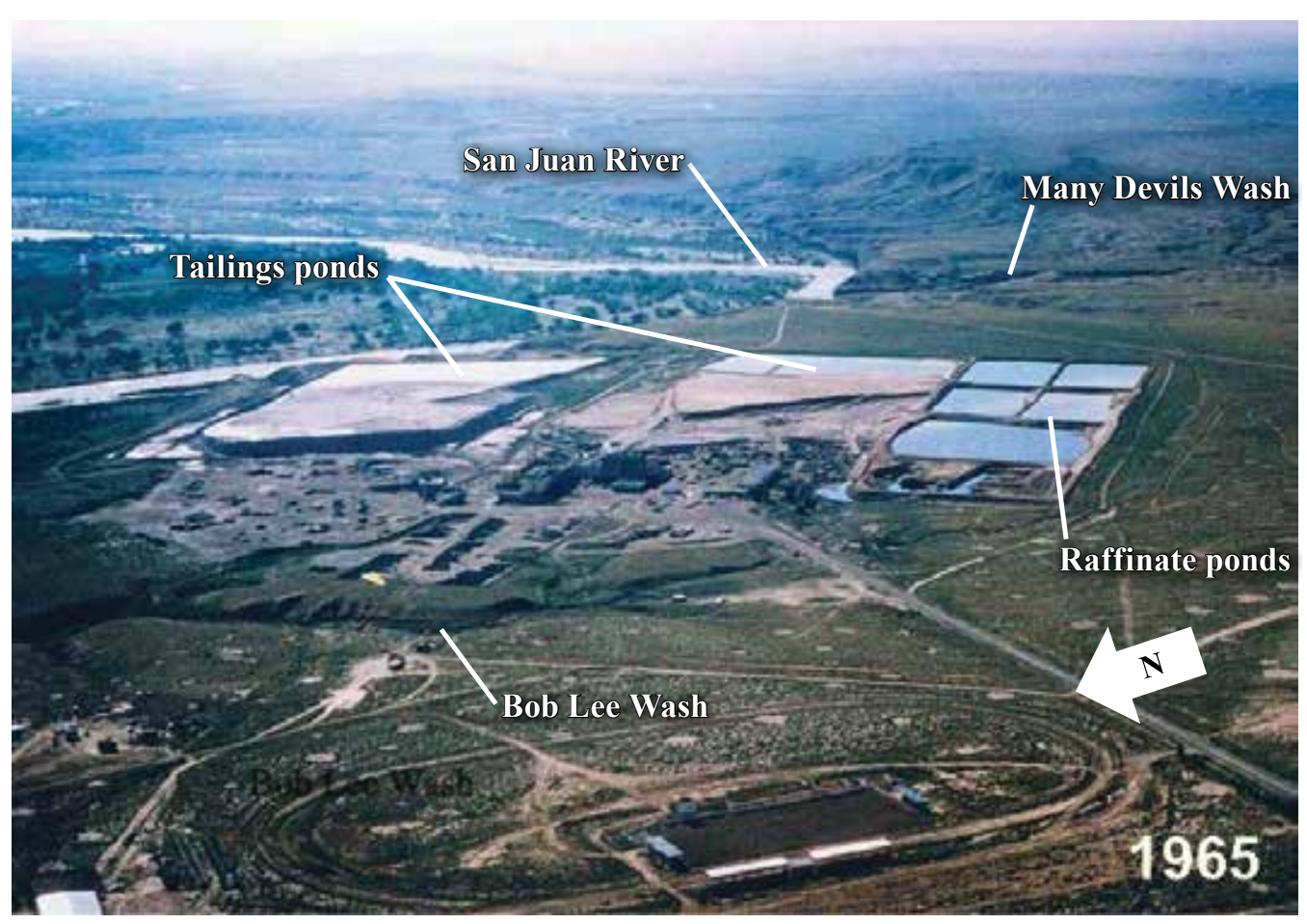

Figure 2. The Navajo Mill, Shiprock, New Mexico, during its operation in 1965 (U.S. Department of Energy, 2011a). 
The DOE estimates that the Mill used about 1 billion gallons of water during active operations, which was pumped to the Mill from the San Juan River (U.S. Department of Energy, 2000). It is likely that much of this water, along with the process constituents, percolated into the subsurface during the Mill operations. The amounts of water and waste products introduced into the subsurface are unknown, but DOE has estimated that between 50 million and 390 million gallons of water were leaked to the subsurface (U.S. Department of Energy, 2012c). In 1960, the Public Health Service (1962) performed a water-quality stream survey of the San Juan River near the Mill after a ruptured pond accidentally released raffinate waste. A report from that survey provided the following description (Public Health Service, 1962, p. 3) of the waste process and groundwater:

"The portions of the ponded liquids which do not evaporate normally percolate into a 10 to 20 foot thick terrace gravel deposit which is underlain by Mancos Shale. These liquids then flow laterally over the shale to the edge of the bluff, where the shale crops out from 10 to $20 \mathrm{ft}$ above the narrow flood plain of the river. The major portion of the seepage appears in various gullies and washes where the alluvium has been eroded from the bluff."

After the Mill closed, the facility was decommissioned and razed. Remedial actions to stabilize surface and nearsurface contamination were completed in 1986. As part of this remediation, the two tailings piles were combined and stabilized onsite in a disposal cell that covers 76 acres and contains approximately 2,520,000 wet tons (approximately 1.9 million cubic yards) of process-related materials (U.S. Department of Energy, 2012c). The disposal cell was designed to encapsulate and isolate the contaminated material for 200 to 1,000 years. The cell cover is constructed of a thick radon barrier composed of windblown clayey and silty soil (loess) derived mainly from Mancos Shale and an outer erosionprotection layer of resistant rock cobbles. The radon barrier is designed to reduce radon emissions and to promote the rapid runoff of precipitation (U.S. Department of Energy, 2011c).

Groundwater monitoring began in the 1980s, and current groundwater-remediation efforts include institutional controls, such as fencing and signage, natural attenuation, and groundwater capture (U.S. Department of Energy, 2011c). Active remediation of groundwater at the Shiprock Disposal Site was initiated in March 2003 and was designed to remove Mill-affected groundwater from the subsurface through extraction wells and interceptor drains. The groundwater that is collected from the wells and drains is pumped to an evaporation pond, located to the south of the disposal cell.

\section{Regional Geology}

The Shiprock Disposal Site is located on the Four Corners platform in the San Juan Basin (fig. 1), the dominant geologic feature in the northwestern part of New Mexico (Craigg, 2001).

\section{Structure}

The San Juan structural basin is a northwest-trending, asymmetric structural depression with an area of about 21,600 square miles $\left(\mathrm{mi}^{2}\right)$ at the eastern edge of the Colorado Plateau (Levings and others, 1996). The basin formed during the Laramide orogeny in the Late Cretaceous to Early Tertiary periods (Dam, 1995) and contains a thick sequence (more than $2 \frac{1}{2}$ mi thick) of nearly horizontal sedimentary Paleozoic, Mesozoic, and Cenozoic rocks overlying a Precambrian basement complex (Levings and others, 1996). The basin is bounded by the San Juan uplift, La Plata Mountains, and Sleeping Ute Mountain to the north; the Carrizo and Chuska Mountains and Defiance uplift to the west; the Zuni uplift to the south; the Lucero uplift and Ignacio monocline to the southeast; and the Nacimiento uplift and the Gallina-Archuleta arch to the east (fig. 1) (Brister and Hoffman, 2002).

\section{Stratigraphy}

The sedimentary strata of the San Juan Basin dip slightly down toward the center of the basin from the surrounding highlands. Older sedimentary and Precambrian rocks (1,500 to 1,750 million years old) are exposed in the uplifts on the outer boundary of the basin (Brister and Hoffman, 2002). Above the Precambrian rocks are the rocks of the lower Paleozoic (fig. 3). The rocks of the Triassic period (primarily the Chinle Group), overlying the Permian strata, were formed from deposits in various continental and fluvial environments and consist of variegated claystone and shale; siltstone; conglomerate; and limestone. Following deposition of Triassic sediments, erosion resulted in a widespread unconformity between the Triassic and Jurassic rocks. The lowermost Jurassic rocks, primarily the Entrada Sandstone, are sandstones that originated as dune fields. Uplift and volcanic activity to the southwest coincided with the deposition of the middle and upper Jurassic Morrison Formation (fig. 3). The Morrison Formation depositional environment was a vast plain with a variety of fluvial and lacustrine environments with evidence of volcanic debris. The Dakota Sandstone unconformably overlies the Morrison Formation throughout much of the basin and ranges from zero to about $500 \mathrm{ft}$ thick, with 200 to $300 \mathrm{ft}$ being the most common (Dam, 1995). The Dakota Sandstone is conformably overlain by the Mancos Shale, with sandstone intertonguing common (Dam, 1995). The Mancos Shale was deposited during the Late Cretaceous (about 95 to 65 million years ago), when the western United States was dissected by the large Cretaceous Interior Seaway with a moving shoreline that deposited about $6,500 \mathrm{ft}$ of marine, coastal plain, and nonmarine sediments including shales, mudstones, limestones, sandstones (such as the Gallup Sandstone), coal, and conglomerates (Brister and Hoffman, 2002). The Cretaceous shale members represent deeper 


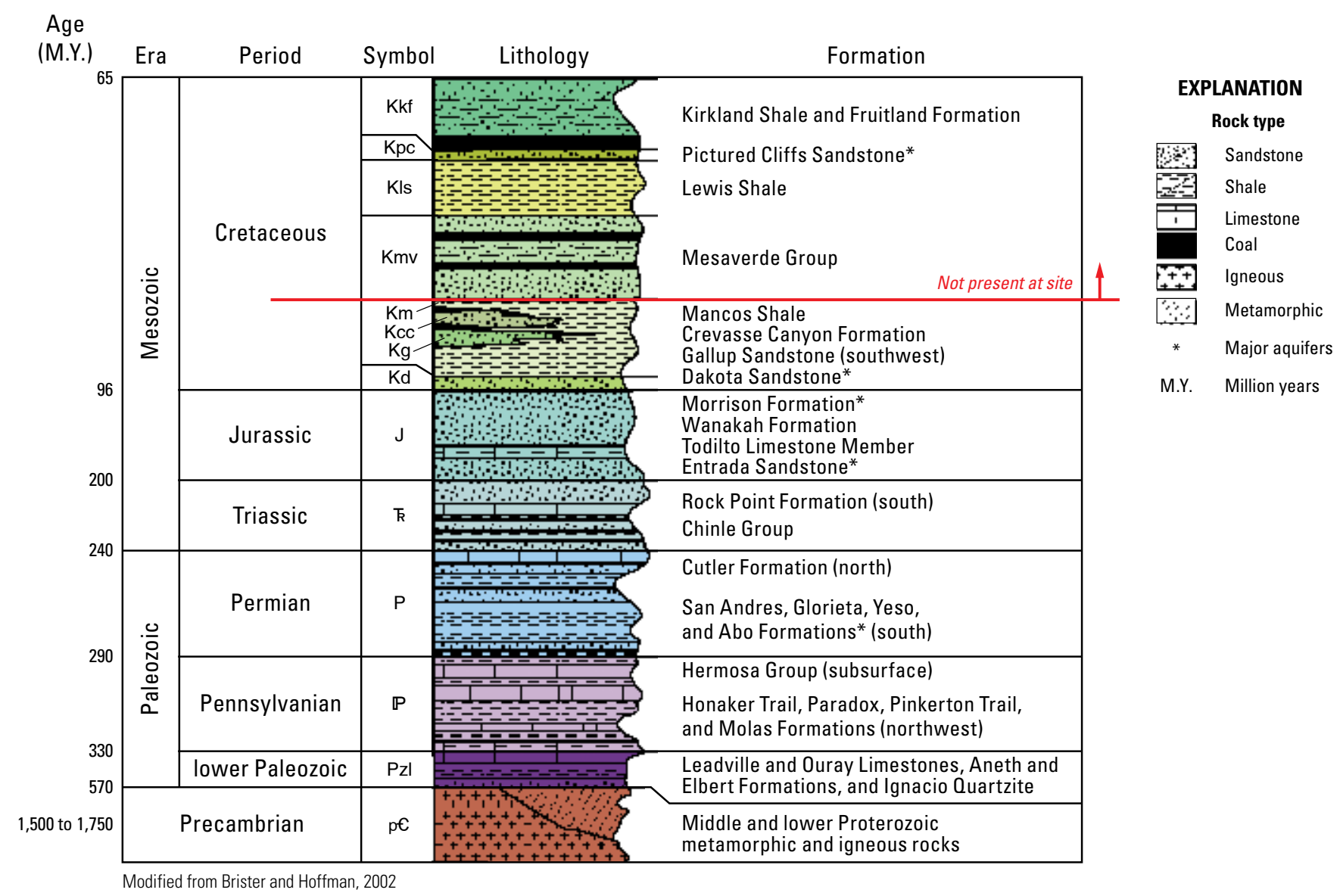

Figure 3. Stratigraphic column of the San Juan Basin, New Mexico.

and less energetic nearshore (toe-of-slope) environments (Krumholz, 1997). The upper Cretaceous and Tertiary layers are absent in this part of the basin because of uplift and erosion (Levings and others, 1996).

The lithologic log of the flowing well 648 , located just west of the disposal cell (fig. 4), records the stratigraphy underlying the site. The well is completed as an open hole from 1,482 to $1,777 \mathrm{ft}$ below land surface (bls). According to the lithologic log, there is $30 \mathrm{ft}$ of alluvium overlying $218 \mathrm{ft}$ of the upper Mancos Shale. The upper Mancos Shale is underlain by $82 \mathrm{ft}$ of Gallup Sandstone, which is underlain by $685 \mathrm{ft}$ of the lower Mancos Shale, followed by $165 \mathrm{ft}$ of Dakota sandstone. The Morrison Formation begins at 1,180 ft bls and ends at 1,760 ft bls. The sandstone described in the $\log$ as the Gallup Sandstone may be an isolated lenticular sandstone body known as Tocito Sandstone Lentils that has been misidentified as part of the Gallup Sandstone (Kernodle, 1996). Although Tocito Sandstone Lentils are found at similar stratigraphic positions as the Gallup Sandstone, they are not related. The mapping of the Gallup Sandstone during the
USGS Regional Aquifer-System Analysis (RASA) study projected the Gallup Sandstone to be truncated just south of Shiprock because of late Cretaceous erosion (Levings and others, 1996). This projected extent, however, does not preclude the existence of the Gallup Sandstone in the project area and should be regarded with caution. In this report, the sandstone identified in the log for well 648 is referred to as the "Gallup Sandstone.

\section{Lithology and Mineralogy}

The Mancos Shale directly underlies the Shiprock Disposal Site and the surrounding area, including Many Devils Wash (Ward, 1990; U.S. Department of Energy, 2000). On the terrace, the Mancos Shale is overlain by unconsolidated Quaternary alluvial deposits derived from the ancestral San Juan River and more recent deposits of loess. The unconsolidated Quaternary deposits in the Many Devils Wash watershed include alluvium, colluvium, and loess. 


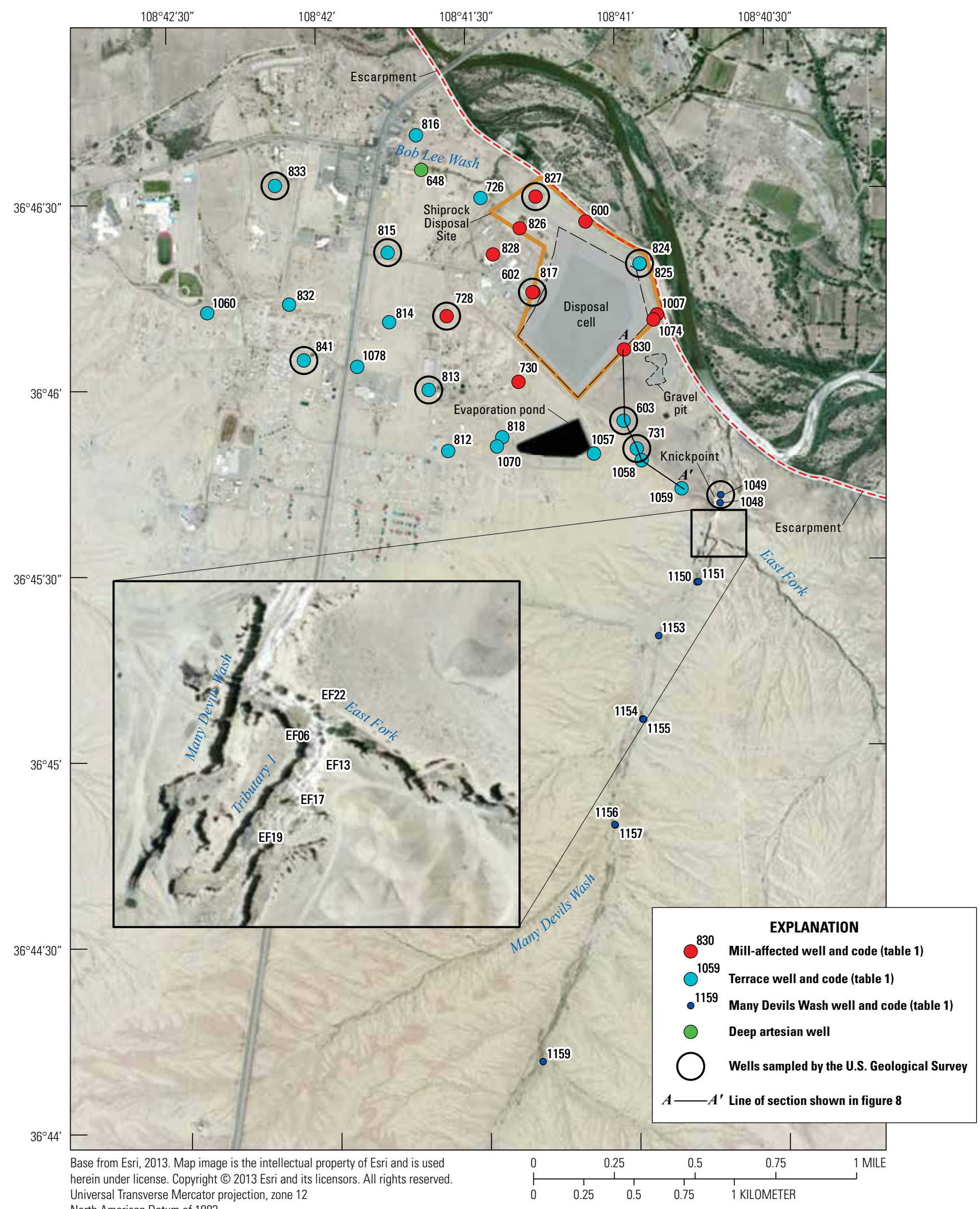

Figure 4. Well locations and sampling sites at and near the Shiprock Disposal Site, Shiprock, New Mexico, and line of section shown in figure 8. 
The Source of Groundwater and Solutes to Many Devils Wash at a Former Uranium Mill Site in Shiprock, New Mexico

\section{Mancos Shale}

The Mancos Shale is widely distributed throughout northwestern New Mexico, western Colorado, and eastern Utah and is correlative to the Cretaceous Pierre Shale in eastern Colorado (Holloway and Smith, 2005). It generally consists of clayey to sandy to calcareous silt-shale with minor limestone, marlstone, bentonite, concretions, and sandstone (U.S. Department of Energy, 2000; Ball and others, 2010). These Late Cretaceous shales are described as organic-carbon and sulfide enriched (McMahon and others, 1999). Mineralogy of bulk samples from the Mancos Shale at the Mars Desert Research Station in Utah contained hydrated sulfate minerals and phyllosilicates (Kotler and others, 2011). Clay fractions analyzed by x-ray diffraction and Fourier transform infrared spectroscopy were illite-smectites, with the smectites identified as montmorillonite and nontronite (Kotler and others, 2011). The most common sulfate mineral was gypsum, and detected carbonates included calcite, dolomite, ankerite, and siderite (Kotler and others, 2011).

About $900 \mathrm{ft}$ of the Mancos Shale underlies the Shiprock Disposal Site, and it generally is light gray to dark gray and calcareous throughout, especially in the lighter-colored, coarser-grained (silty) layers (U.S. Department of Energy, 2000). Thin claystone layers (up to several inches thick) are common and are dark gray; they swell when brought to the surface and are likely excellent aquicludes (confining units).
Traces of carbonaceous material and finely disseminated pyrite were identified in cores collected from boreholes drilled near the site (U.S. Department of Energy, 2000). Thin bentonite (clays composed mostly of montmorillonite produced by the alteration of volcanic ash) beds ranging from a fraction of an inch (in.) to as much as $5 \mathrm{ft}$ in thickness, but most commonly 1 to 6 in. in thickness, have been identified in the Mancos Shale (U.S. Department of Energy, 2000).

A distinguishing feature of the Mancos Shale at the site is the presence of a continuous, distinctive, thin, tanto-orange siltstone bed about $1 \mathrm{ft}$ thick. The bed is exposed in the escarpment cliff north and east of the disposal cell, starting from the area near the disposal cell and extending southeastward along the San Juan River to about 1,000 ft east of the confluence of Many Devils Wash, where the bed dips below the flood plain of the river. The position of the siltstone bed on the escarpment gradually drops in elevation from its westernmost exposure to its easternmost exposure, following the reported low-angle dip (about 0.5 degrees to the east) of the Mancos Shale (U.S. Department of Energy, 2000). The siltstone subcrops below the disposal cell and is absent to the west on the basis of projections from well logs. The same siltstone bed is exposed in the downstream part of Many Devils Wash where it forms a knickpoint (a sharp change in slope caused by erosion) in the channel (fig. 5) about $1,200 \mathrm{ft}$ upstream from the confluence with the San Juan River.

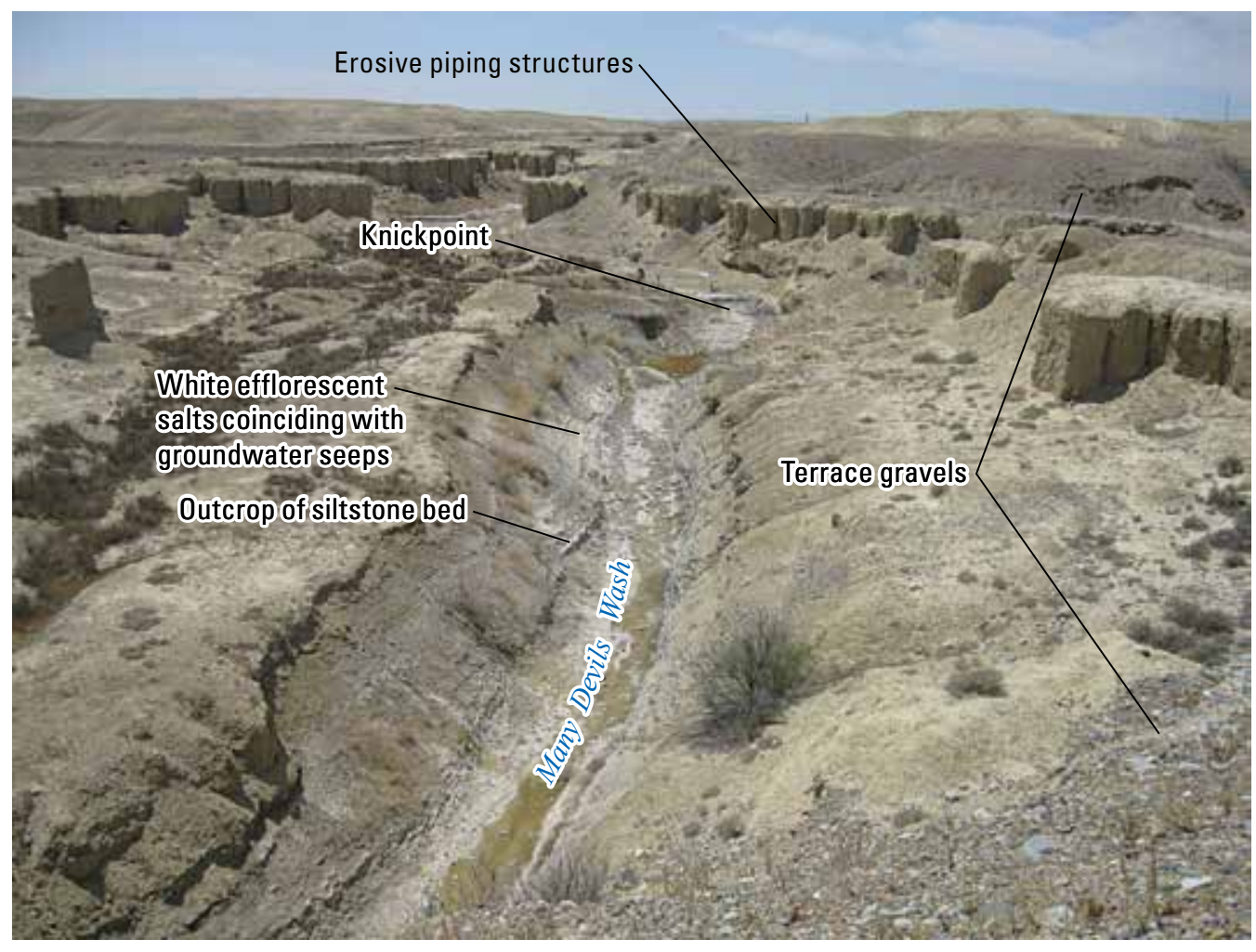

Figure 5. Efflorescent deposits in Many Devils Wash at the Shiprock Disposal Site, New Mexico, looking upstream (south) at the knickpoint and showing the outcrop of the siltstone bed and terrace gravels (U.S. Geological Survey). 


\section{Terrace Sediments}

The unconsolidated sediments overlying the Mancos Shale on the terrace consist of alluvial deposits from ancestral San Juan River glacial outwash and windblown sediments (loess) (U.S. Department of Energy, 2011a). The alluvial sediments were deposited during aggradation in an ancestral San Juan River Valley during the late part of the Bull Lake glaciation (Late to Middle Pleistocene), 88,000 to 150,000 years ago (Tsosie, 1997). Later erosion and downcutting have left remnants of these deposits preserved on strath terraces. Clast-supported deposits of well-rounded gravel, cobbles, and boulders with a silty and sandy matrix compose much of the terrace deposits (fig. 5). The coarsest part of the deposit is typically at the base, where cobbles $1 \mathrm{ft}$ in diameter are common and have been found to be as much as $2.5 \mathrm{ft}$ in diameter (U.S. Department of Energy, 2000). The resistant cobbles and boulders typically consist of metamorphic rocks (quartzite and metaconglomerate) likely eroded from the San Juan Mountains associated with the San Juan uplift in southwestern Colorado. Locally, the terrace alluvium also can contain less-coarse sediments and more angular debris derived from nearby tributaries. These sediments are typically 10 to $20 \mathrm{ft}$ thick where exposed along the top of the escarpment (adjacent to the disposal cell).

Eolian loess deposits overlie the glacial outwash sands and gravels on the terrace south of the disposal cell and lie directly on the Mancos Shale within much of the watershed of Many Devils Wash. The loess is composed mainly of silt, with minor amounts of very fine-grained sand, clayey silt, and sandy clay (U.S. Department of Energy, 2011a). Much of the loess was derived from ablation of extensive Mancos Shale areas to the south and west. In places, thin layers of coarse-grained sand and small pebbles occur, likely indicating episodes of fluvial erosion and deposition. Erosion in the upstream areas of the Many Devils Wash is actively incising through the loess.

Deposits of white salts, known as efflorescent crusts, of variable thicknesses are present on outcrops of Mancos Shale along the escarpment and in Many Devils Wash (fig. 5). Efflorescent crusts have been reported as evidence of groundwater discharge zones by Butler and others (1996). In their work describing the efflorescent crusts associated with the Mancos Shale, Whittig and others (1982) report extensive mineral species identified from the deposits collected near seeps issuing from the Mancos Shale.

The efflorescent crusts in Many Devils Wash occur on the wash bottom (fig. 5) and on the east bank for most of the distance from the confluence with the San Juan River to the confluence with the East Fork. The crusts extend only about $150 \mathrm{ft}$ upstream on the East Fork and are absent upstream from the confluence with Tributary 1 (fig. 4). They are not present in the main channel of Many Devils Wash where it parallels Tributary 1 to the west. Infrequent rains dissolve the crust, but it reappears after several days of dry conditions. This process was observed in Many Devils Wash on March 28, 2000, by the DOE, when the crust disappeared after approximately a 0.75 -in. rain. Dry conditions reestablished the crust within the following week (U.S. Department of Energy, 2000).

\section{Climate and Vegetation}

The Shiprock Disposal Site is located in the arid Southwestern United States and typically has mild winters with periodic cold-front storms; hot, dry, and windy springs and early summers; warm and monsoonal late summers; and cool, clear autumns (Levings and others, 1996). The basin is climatically arid, and vegetation near the site primarily consists of treeless shrub grasslands (Hall, 1990). The mean annual temperature in Shiprock is 55.3 degrees Fahrenheit $\left({ }^{\circ} \mathrm{F}\right)$, and the mean annual precipitation is $7.0 \mathrm{in}$. (Western Regional Climate Center, 2012). More than half of the annual precipitation falls from July through October (Hall, 1990). Potential annual evaporation is more than 60 in., greatly exceeding precipitation (Levings and others, 1996). With additional losses to transpiration, the potential annual water deficit is large; however, timing of precipitation allows for periodic runoff and recharge (Levings and others, 1996).

\section{Surface Hydrology}

The study area is drained by the San Juan River with an average annual flow (from 1935 to 2013) at the USGS streamgage at Shiprock (09368000) of 1,983 cubic feet per second ( $\mathrm{ft}^{3} / \mathrm{s}$ ) (http://dx.doi.org/10.5066/F7P55KJN). The Navajo Reservoir was completed in 1963 on the San Juan River approximately 78 mi upstream from the Shiprock Disposal Site (http:/www.usbr.gov/uc/rm/crsp/navajo/). The reservoir supplies water for agricultural and municipal uses. Upstream from the reservoir, a part of the water is diverted into the Rio Grande Basin as part of the Colorado River Compact appropriations. The La Plata and Animas Rivers are two major tributaries that contribute unregulated flow into the San Juan River upstream from the site.

The terrace at the Shiprock Disposal Site is trisected by two prominent arroyos, Bob Lee Wash and Many Devils Wash, which both drain to the San Juan River (figs. 2 and 4). These two arroyos may be considered perennial because of groundwater contributions. Discharge from the flowing well 648 , at approximately 64 gallons per minute ( $\mathrm{gal} / \mathrm{min}$ ), is responsible for most, if not all, of the flow in Bob Lee Wash (U.S. Department of Energy, 2000). Many Devils Wash is supplied by numerous small seeps located in the northernmost (downstream) end of the wash. The discharge to Many Devils Wash from groundwater is small $(0.3 \mathrm{gal} / \mathrm{min}$ measured in March 1999) and may vary depending upon recent recharge and evaporation (U.S. Department of Energy, 2000). Pools of yellow- to red-colored water are common in the stream channel of Many Devils Wash and contain high concentrations of $\mathrm{NO}_{3}, \mathrm{Se}, \mathrm{SO}_{4}$, and U (U.S. Department of Energy, 2000). 


\section{Groundwater Hydrology}

Regional aquifers in the San Juan Basin are coincident with the permeable geologic units (Levings and others, 1996). The major aquifers underlying the site are found in the Dakota Sandstone, the Morrison Formation, the Entrada Sandstone, and the San Andres and Glorieta Formations (Dam, 1995). The Gallup Sandstone is considered a regional aquifer but may be missing at the site because of post-deposition erosion (see discussion in the "Regional Geology" section). Thick shale beds of the Cretaceous and Triassic periods act as confining layers between these aquifers. Vertical leakage between aquifers is thought to occur, but the magnitude is considered small (Dam, 1995). Recharge to these regional aquifers occurs along the outcrops that form the structural boundaries of the basin, and the general flow directions from these recharge points are to the San Juan River (Dam, 1995). Water enters the groundwater-flow system from precipitation on aquifer outcrops and from stream-channel loss as streams cross the outcrops (Craigg, 2001).

The Mancos Shale is not generally considered an aquifer because of its low hydraulic conductivity. Waters associated with the Mancos Shale are typically described as occurring in an adjacent aquifer and infiltrating into the formation to a discrete depth. The alluvium at the site has a water table, but the productivity of many wells screened in the alluvium is very small (U.S. Department of Energy, 2000).

\section{Previous Studies}

Numerous reports, beginning in the 1800s, describe San Juan Basin hydrology, geology, and water chemistry. Early reports were compiled in a bibliographic reference published in 1979 (Wright, 1979). Between 1984 and 1990, the USGS conducted a regional assessment of the water-bearing units in the San Juan Basin as part of the RASA program (Levings and others, 1996).

Several investigations beginning in the late 1970s have studied the contributions of salinity in the Colorado River from the Mancos Shale (Laronne and Schumm, 1977; Wagenet and Jurinak, 1978; Duffy and others, 1985; Azimi-Zonooz and Duffy, 1993). These previous investigations concluded that weathering of the Mancos Shale is the dominant process controlling major-ion chemistry in groundwater or surface water that is in contact with the Mancos Shale. Based on an analysis of water extracts from samples of weathered and unweathered Mancos Shale collected from the West Salt Creek watershed near Grand Junction in western Colorado,
Evangelou and others (1984) stated that the calcium-sulfate composition of groundwater in contact with the weathered Mancos Shale results from the dissolution of calcite, dolomite, and gypsum. They also concluded that the unweathered shale contains very little, if any, gypsum and that the high concentrations of soluble sodium $(\mathrm{Na})$ in the unweathered Mancos Shale results from the exchange of calcium $(\mathrm{Ca})$ for $\mathrm{Na}$ on the clay adsorption sites. More recently, Tuttle and Grauch (2009) and Tuttle and others (2014) have expanded on the weathering processes of the Mancos Shale to include weathering of iron sulfide $\left(\mathrm{FeS}_{2}\right)$ minerals that oxidize to form aqueous sulfates. Weathering also affects organic material in the Mancos Shale. On the basis of depth-dependent sampling of the Mancos Shale in Utah, Leythaeuser (1973) determined that there was less organic content and that the organic composition had a lower fraction of hydrocarbons in the shallow samples, relative to samples collected at depth due to increased weathering.

In the early 1980s, researchers discovered that Se in Kesterson Reservoir, California, was detrimentally affecting fish and waterfowl, and that the Se was derived from irrigation drainage in areas underlain by Cretaceous marine shales (Presser, 1994). Following the "Kesterson Effect" (Presser, 1994), a number of investigations focused on the relation of $\mathrm{Se}$ and other constituents to irrigation in certain areas, especially those areas on Mancos Shale. The response was a multiagency program known as the National Irrigation Water Quality Program that produced a number of reports at sites throughout the upper Colorado River watershed (U.S. Department of Interior, 2001). Two of those studies (Blanchard and others, 1993; Thomas and others, 1998) focused on the San Juan River area.

Numerous site-specific hydrogeologic studies have been completed at the Shiprock Disposal Site by the U.S. Public Health Service and by DOE and its contractors. Data collected from these investigations are used and cited throughout this report.

\section{Study Methods}

The majority of the data analyzed in this report were collected by DOE and were obtained from their Geospatial Environmental Mapping System (GEMS) database (http:// gems.Im.doe.gov/\#\&site=SHP; U.S. Department of Energy, 2014) or various DOE publications referenced throughout this report. A list of wells and sample sites and the type of data used in this report are provided in table 1. 
Table 1. Shiprock Disposal Site well information and selected sample data, Shiprock, New Mexico.

$\left[\mathrm{AR},{ }^{234} \mathrm{U} /{ }^{238} \mathrm{U}\right.$ (uranium isotope) alpha activity ratio; $\delta^{18} \mathrm{O}$, isotopic composition of oxygen-18 in per mil relative to Vienna Standard Mean Ocean Water; $\delta \mathrm{D}$, isotopic composition of deuterium in per mil relative to Vienna Standard Mean Ocean Water; TU, tritium units; SC, specific conductivity; $\mu \mathrm{S} / \mathrm{cm}$ at $25^{\circ} \mathrm{C}$, microsiemens per centimeter at 25 degrees Celsius; $\mathrm{Ca}$, calcium; mg/L, milligrams per liter; $\mathrm{Mg}$, magnesium; K, potassium; SCW, Salt Creek Wash; DOE, Department of Energy; <, less than; UENAS, Upper Eagle Nest Arroyo; $\mathrm{Na}$, sodium; $\mathrm{HCO}_{3}$, bicarbonate alkalinity as $\mathrm{CaCO}_{3} ; \mathrm{Cl}_{1} \mathrm{chloride}$, $\mathrm{SO}_{4}$, sulfate; $\mathrm{NO}_{3}$, nitrate as nitrate; $\mathrm{U}$, uranium; Se, selenium; $\mu \mathrm{g} / \mathrm{L}$, micrograms per liter; $\delta^{34} \mathrm{~S}_{\text {suffate }}$, composition of ${ }^{34} \mathrm{~S}$ in sulfate in per mil relative to Vienna Canyon Diablo Troilite; EF, East Fork of Many Devils Wash; GEMS, Geospatial Environmental Mapping System; -, not reported; n/a, not available]

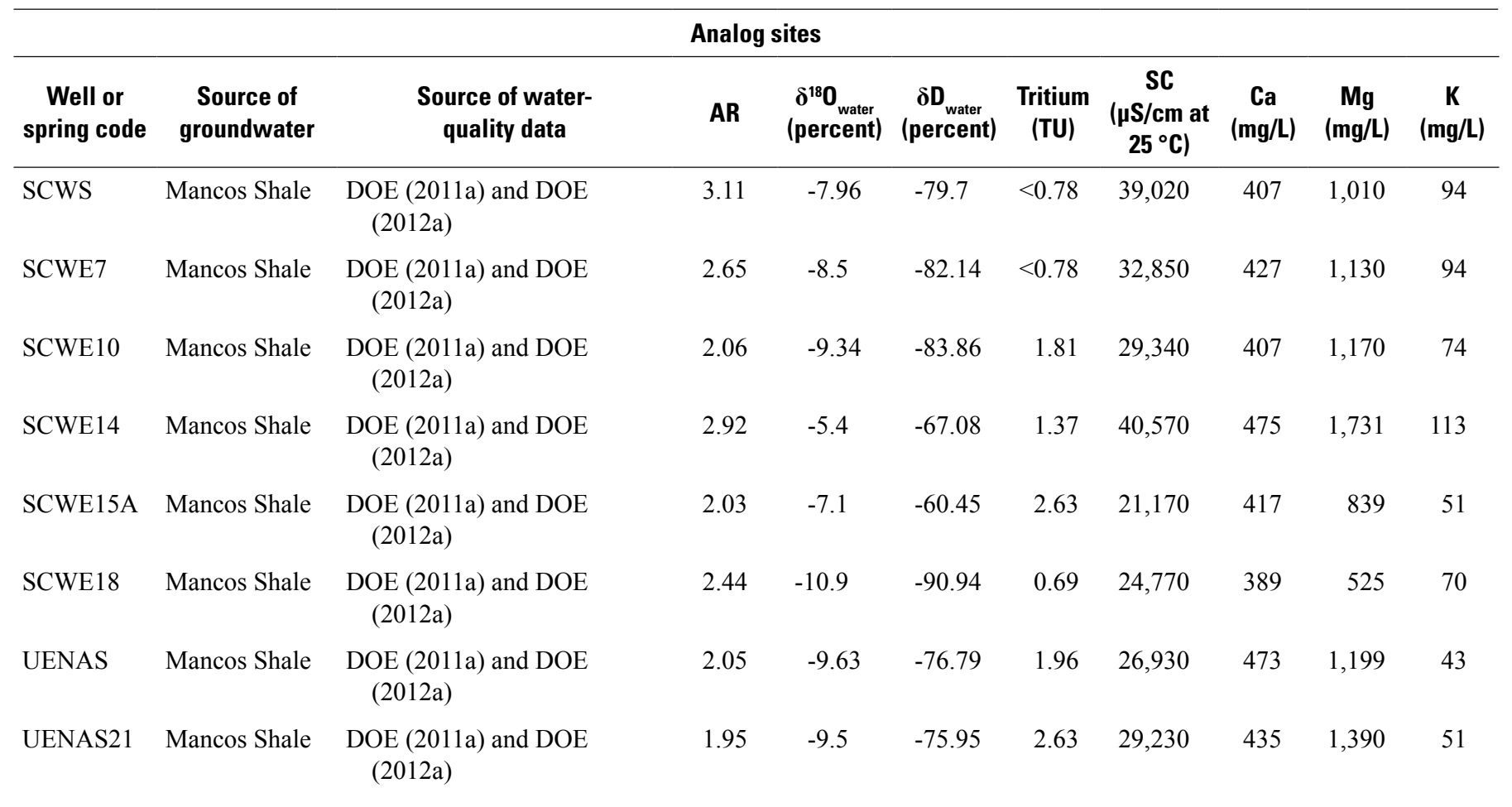

\begin{tabular}{|c|c|c|c|c|c|c|c|c|c|c|}
\hline \multicolumn{11}{|c|}{ Analog sites } \\
\hline $\begin{array}{l}\text { Well or } \\
\text { spring code }\end{array}$ & $\begin{array}{c}\text { Source of } \\
\text { groundwater }\end{array}$ & $\begin{array}{l}\text { Source of water- } \\
\text { quality data }\end{array}$ & $\underset{(\mathrm{mg} / \mathrm{L})}{\mathrm{Na}}$ & $\begin{array}{c}\mathrm{HCO}_{3} \\
\text { (mg/L) }\end{array}$ & $\begin{array}{c}\mathrm{CI} \\
(\mathrm{mg} / \mathrm{L})\end{array}$ & $\begin{array}{c}\mathrm{SO}_{4} \\
(\mathrm{mg} / \mathrm{L})\end{array}$ & $\begin{array}{c}\mathrm{NO}_{3} \\
(\mathrm{mg} / \mathrm{L})\end{array}$ & $\underset{(\mathrm{mg} / \mathrm{L})}{\mathrm{U}}$ & $\begin{array}{c}\mathrm{Se} \\
(\mu \mathrm{g} / \mathrm{L})\end{array}$ & $\begin{array}{c}\delta^{34} S_{\text {sulfate }} \\
\text { (percent) }\end{array}$ \\
\hline SCWE7 & Mancos Shale & $\begin{array}{l}\text { DOE (2011a) and DOE } \\
\quad(2012 a)\end{array}$ & 9,081 & 1,178 & 1,961 & 20,202 & 1,668 & 0.135 & 2,390 & -20.34 \\
\hline SCWE14 & Mancos Shale & $\begin{array}{l}\text { DOE (2011a) and DOE } \\
\quad(2012 a)\end{array}$ & 10,391 & 1,446 & 4,988 & 21,499 & 2,338 & 0.171 & 2,560 & -22.01 \\
\hline SCWE15A & Mancos Shale & $\begin{array}{l}\text { DOE (2011a) and DOE } \\
\quad(2012 a)\end{array}$ & 5,012 & 720 & 1,450 & 10,802 & 1,519 & 0.133 & 1,490 & -22.58 \\
\hline UENAS & Mancos Shale & $\begin{array}{l}\text { DOE (2011a) and DOE } \\
\quad(2012 a)\end{array}$ & 6,161 & 403 & 3,871 & 12,099 & 837 & 0.0452 & 336 & -20.49 \\
\hline UENAS21 & Mancos Shale & $\begin{array}{l}\text { DOE (2011a) and DOE } \\
\quad(2012 a)\end{array}$ & 6,943 & 342 & 4,389 & 13,300 & 874 & 0.0536 & 249 & -19.82 \\
\hline
\end{tabular}


Table 1. Shiprock Disposal Site well information and selected sample data, Shiprock, New Mexico._Continued

$\left[\mathrm{AR},{ }^{234} \mathrm{U} /{ }^{238} \mathrm{U}\right.$ (uranium isotope) alpha activity ratio; $\delta^{18} \mathrm{O}$, isotopic composition of oxygen-18 in per mil relative to Vienna Standard Mean Ocean Water; $\delta \mathrm{D}$, isotopic composition of deuterium in per mil relative to Vienna Standard Mean Ocean Water; TU, tritium units; SC, specific conductivity; $\mu \mathrm{S} / \mathrm{cm}$ at $25^{\circ} \mathrm{C}$, microsiemens per centimeter at 25 degrees Celsius; $\mathrm{Ca}$, calcium; mg/L, milligrams per liter; $\mathrm{Mg}$, magnesium; K, potassium; SCW, Salt Creek Wash; DOE,

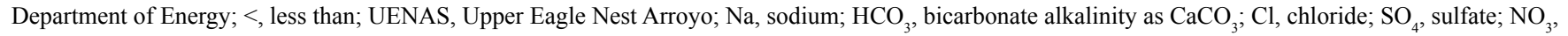
nitrate as nitrate; $\mathrm{U}$, uranium; Se, selenium; $\mu \mathrm{g} / \mathrm{L}$, micrograms per liter; $\delta^{34} \mathrm{~S}_{\text {sulfate }}$, composition of ${ }^{34} \mathrm{~S}$ in sulfate in per mil relative to Vienna Canyon Diablo Troilite; EF, East Fork of Many Devils Wash; GEMS, Geospatial Environmental Mapping System; -, not reported; n/a, not available]

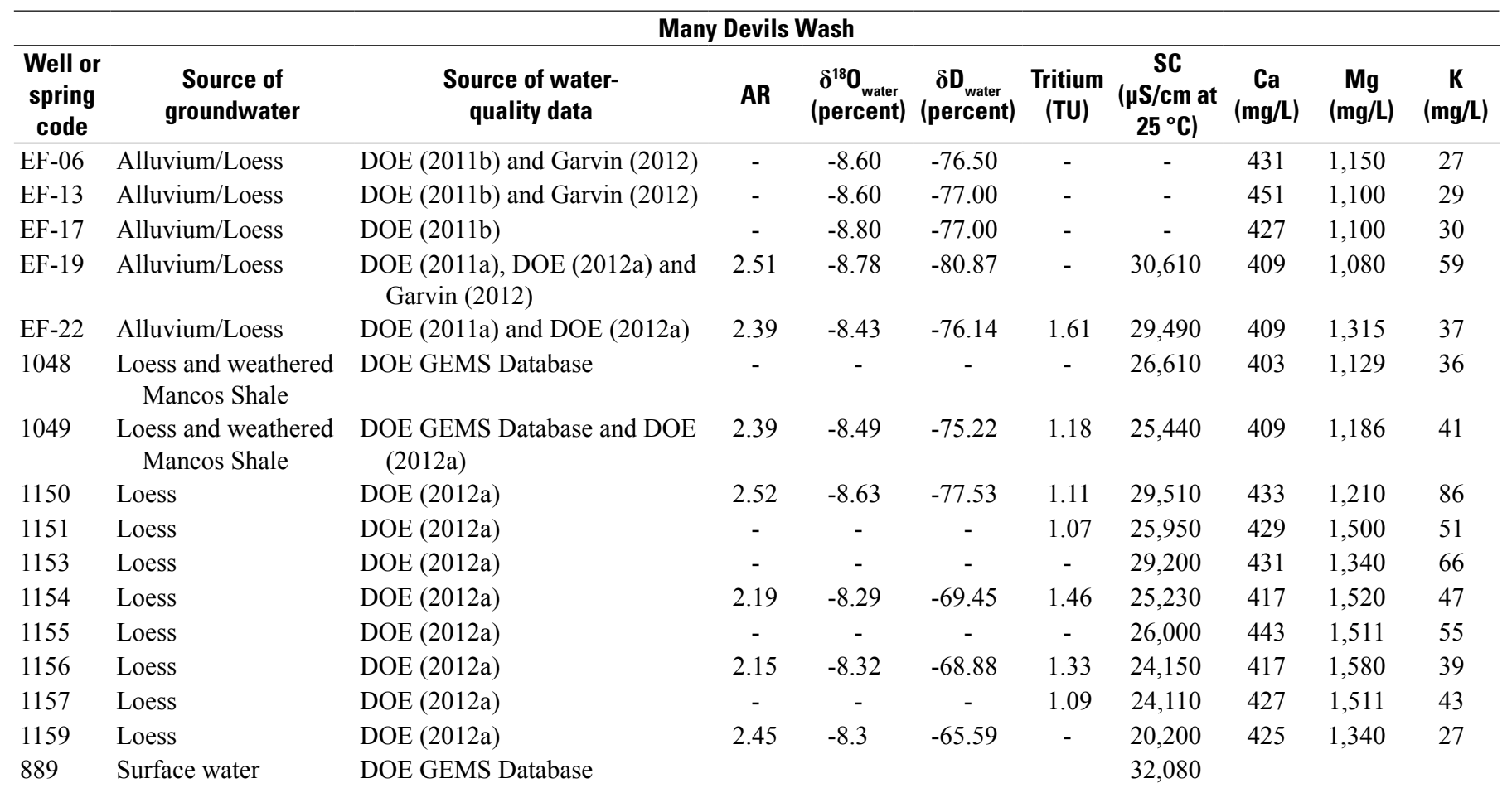

\begin{tabular}{|c|c|c|c|c|c|c|c|c|c|c|}
\hline \multicolumn{11}{|c|}{ Many Devils Wash } \\
\hline $\begin{array}{c}\text { Well or } \\
\text { spring } \\
\text { code }\end{array}$ & $\begin{array}{l}\text { Source of } \\
\text { groundwater }\end{array}$ & $\begin{array}{l}\text { Source of water- } \\
\text { quality data }\end{array}$ & $\underset{(\mathrm{mg} / \mathrm{L})}{\mathrm{Na}}$ & $\begin{array}{l}\mathrm{HCO}_{3} \\
(\mathrm{mg} / \mathrm{L})\end{array}$ & $\begin{array}{c}\mathrm{Cl} \\
(\mathrm{mg} / \mathrm{L})\end{array}$ & $\underset{(\mathrm{mg} / \mathrm{L})}{\mathrm{SO}_{4}}$ & $\underset{(\mathrm{mg} / \mathrm{L})}{\mathrm{NO}_{3}}$ & $\underset{(\mathrm{mg} / \mathrm{L})}{\mathrm{U}}$ & $\underset{(\mu \mathrm{g} / \mathrm{L})}{\mathrm{Se}}$ & $\begin{array}{c}\delta^{34} S_{\text {sulfate }} \\
\text { (percent) }\end{array}$ \\
\hline EF-13 & Alluvium/Loess & $\begin{array}{l}\text { DOE (2011b) and Garvin } \\
\quad(2012)\end{array}$ & 7,702 & 805 & 1,464 & 17,579 & 2,517 & - & 1,100 & - \\
\hline EF-19 & Alluvium/Loess & $\begin{array}{l}\text { DOE (2011a), DOE (2012a) } \\
\text { and Garvin (2012) }\end{array}$ & 8,552 & 830 & 1,251 & 17,498 & 3,181 & 0.156 & 1,560 & -24.43 \\
\hline $\mathrm{EF}-22$ & Alluvium/Loess & $\begin{array}{l}\text { DOE (2011a) and DOE } \\
\quad(2012 a)\end{array}$ & 7,748 & 790 & 1,595 & 19,208 & 2,710 & 0.160 & 1,620 & -26.55 \\
\hline 1048 & $\begin{array}{l}\text { Loess and weathered } \\
\text { Mancos Shale }\end{array}$ & DOE GEMS Database & 6,920 & 687 & 1,214 & 17,355 & 2,801 & 0.150 & 1,650 & - \\
\hline 1151 & Loess & $\operatorname{DOE}(2012 a)$ & 6,460 & 665 & 1,588 & 16,499 & 1,699 & 0.149 & 820 & - \\
\hline 1153 & Loess & $\operatorname{DOE}(2012 a)$ & 7,357 & 873 & 1,549 & 17,901 & 2,412 & 0.172 & 1,140 & - \\
\hline 1154 & Loess & $\operatorname{DOE}(2012 a)$ & 6,046 & 708 & 1,539 & 15,802 & 1,389 & 0.139 & 627 & -30.66 \\
\hline 1155 & Loess & $\operatorname{DOE}(2012 a)$ & 6,414 & 708 & 1,571 & 16,201 & 1,432 & 0.153 & 453 & - \\
\hline 1156 & Loess & $\operatorname{DOE}(2012 a)$ & 5,656 & 720 & 1,461 & 15,000 & 1,252 & 0.150 & 569 & -30.89 \\
\hline 1157 & Loess & DOE (2012a) & 5,541 & 689 & 1,439 & 15,101 & 1,209 & 0.148 & 556 & - \\
\hline 1159 & Loess & $\operatorname{DOE}(2012 a)$ & 4,368 & 689 & 1,099 & 11,902 & 862 & 0.106 & 388 & -31.14 \\
\hline 889 & Surface water & DOE GEMS Database & & & & & & & & - \\
\hline
\end{tabular}


Table 1. Shiprock Disposal Site well information and selected sample data, Shiprock, New Mexico._Continued

$\left[\mathrm{AR},{ }^{234} \mathrm{U} /{ }^{238} \mathrm{U}\right.$ (uranium isotope) alpha activity ratio; $\delta^{18} \mathrm{O}$, isotopic composition of oxygen-18 in per mil relative to Vienna Standard Mean Ocean Water; $\delta \mathrm{D}$, isotopic composition of deuterium in per mil relative to Vienna Standard Mean Ocean Water; TU, tritium units; SC, specific conductivity; $\mu \mathrm{S} / \mathrm{cm}$ at $25^{\circ} \mathrm{C}$, microsiemens per centimeter at 25 degrees Celsius; Ca, calcium; mg/L, milligrams per liter; $\mathrm{Mg}$, magnesium; K, potassium; SCW, Salt Creek Wash; DOE, Department of Energy; <, less than; UENAS, Upper Eagle Nest Arroyo; $\mathrm{Na}$, sodium; $\mathrm{HCO}_{3}$, bicarbonate alkalinity as $\mathrm{CaCO}_{3} ; \mathrm{Cl}_{1} \mathrm{chloride}$, $\mathrm{SO}_{4}$, sulfate; $\mathrm{NO}_{3}$, nitrate as nitrate; $\mathrm{U}$, uranium; Se, selenium; $\mu \mathrm{g} / \mathrm{L}$, micrograms per liter; $\delta^{34} \mathrm{~S}_{\text {sulfate }}$, composition of ${ }^{34} \mathrm{~S}$ in sulfate in per mil relative to Vienna Canyon Diablo Troilite; EF, East Fork of Many Devils Wash; GEMS, Geospatial Environmental Mapping System; -, not reported; n/a, not available]

\begin{tabular}{|c|c|c|c|c|c|c|c|c|c|c|}
\hline \multicolumn{11}{|c|}{ Mill-affected wells } \\
\hline $\begin{array}{l}\text { Well or } \\
\text { spring } \\
\text { code }\end{array}$ & $\begin{array}{c}\text { Source of } \\
\text { groundwater }\end{array}$ & $\begin{array}{l}\text { Source of water- } \\
\text { quality data }\end{array}$ & AR & $\begin{array}{l}\delta^{18} 0_{\text {water }} \\
\text { (percent) }\end{array}$ & $\begin{array}{c}\delta D_{\text {water }} \\
\text { (percent) }\end{array}$ & $\begin{array}{l}\text { Tritium } \\
\text { (TU) }\end{array}$ & $\begin{array}{c}\text { SC } \\
(\mu \mathrm{S} / \mathrm{cm} \text { at } \\
\left.25^{\circ} \mathrm{C}\right)\end{array}$ & $\begin{array}{c}\mathrm{Ca} \\
(\mathrm{mg} / \mathrm{L})\end{array}$ & $\underset{(\mathrm{mg} / \mathrm{L})}{\mathrm{Mg}}$ & $\begin{array}{c}\mathrm{K} \\
(\mathrm{mg} / \mathrm{L})\end{array}$ \\
\hline 602 & Weathered Mancos Shale & DOE GEMS Database & 1.39 & - & - & - & 20,714 & 423 & 2,514 & 203 \\
\hline 728 & $\begin{array}{l}\text { Alluvium and Weathered } \\
\text { Mancos Shale }\end{array}$ & DOE GEMS Database & 1.08 & - & - & - & 8,290 & 483 & 944 & 91 \\
\hline 817 & Weathered Mancos Shale & $\begin{array}{l}\text { DOE GEMS Database } \\
\text { and DOE (2012a) }\end{array}$ & 0.99 & -8.90 & -78.73 & 19.8 & 19,310 & 478 & 1,804 & 229 \\
\hline 826 & $\begin{array}{l}\text { Alluvium and Weathered } \\
\text { Mancos Shale }\end{array}$ & $\begin{array}{l}\text { DOE GEMS Database } \\
\text { and DOE (2012a) }\end{array}$ & 1.07 & -9.38 & -83.79 & - & 18,030 & 431 & 2,444 & 130 \\
\hline 830 & $\begin{array}{l}\text { Alluvium and Weathered } \\
\text { Mancos Shale }\end{array}$ & DOE GEMS Database & 1.13 & - & - & - & 2,990 & 582 & 48 & 5 \\
\hline 1007 & $\begin{array}{l}\text { Alluvium and Weathered } \\
\text { Mancos Shale }\end{array}$ & $\begin{array}{l}\text { DOE GEMS Database } \\
\text { and DOE (2012a) }\end{array}$ & 1.16 & -8.70 & -80.62 & 27.2 & 17,840 & 462 & 2,173 & 124 \\
\hline 1074 & $\begin{array}{l}\text { Alluvium and Weathered } \\
\text { Mancos Shale }\end{array}$ & $\begin{array}{l}\text { DOE GEMS Database } \\
\text { and DOE (2012a) }\end{array}$ & 1.13 & -6.95 & -75.92 & 19.7 & 18,540 & 569 & 2,157 & 53 \\
\hline
\end{tabular}

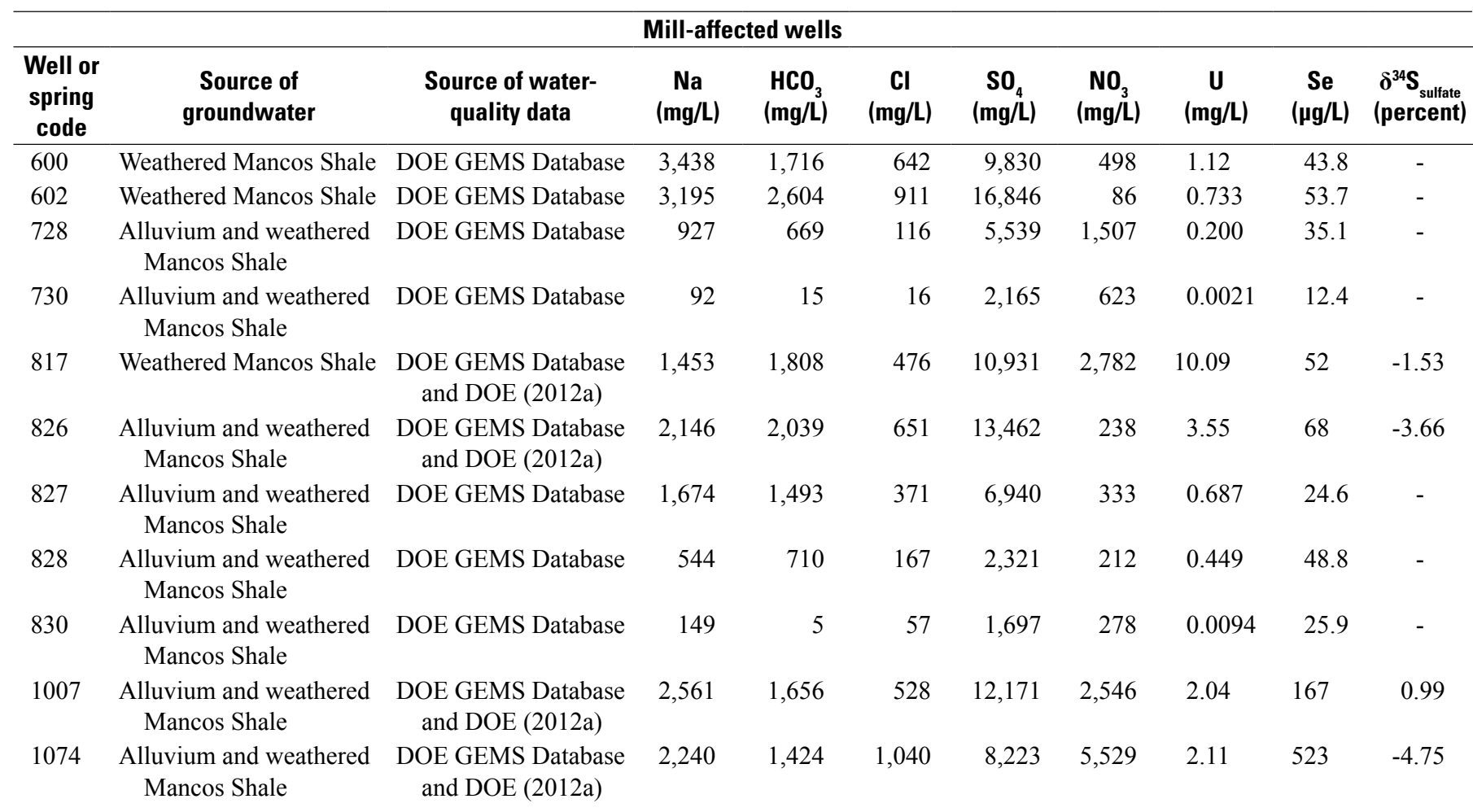




\section{The Source of Groundwater and Solutes to Many Devils Wash at a Former Uranium Mill Site in Shiprock, New Mexico}

Table 1. Shiprock Disposal Site well information and selected sample data, Shiprock, New Mexico.—Continued

$\left[\mathrm{AR},{ }^{234} \mathrm{U} /{ }^{238} \mathrm{U}\right.$ (uranium isotope) alpha activity ratio; $\delta^{18} \mathrm{O}$, isotopic composition of oxygen-18 in per mil relative to Vienna Standard Mean Ocean Water; $\delta \mathrm{D}$, isotopic composition of deuterium in per mil relative to Vienna Standard Mean Ocean Water; TU, tritium units; SC, specific conductivity; $\mu \mathrm{S} / \mathrm{cm}$ at $25^{\circ} \mathrm{C}$, microsiemens per centimeter at 25 degrees Celsius; $\mathrm{Ca}$, calcium; mg/L, milligrams per liter; $\mathrm{Mg}$, magnesium; K, potassium; SCW, Salt Creek Wash; DOE,

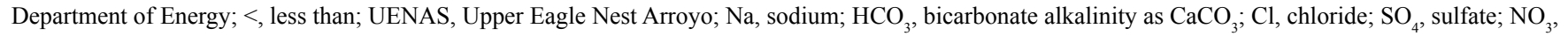
nitrate as nitrate; $\mathrm{U}$, uranium; Se, selenium; $\mu \mathrm{g} / \mathrm{L}$, micrograms per liter; $\delta^{34} \mathrm{~S}_{\text {sulfate }}$, composition of ${ }^{34} \mathrm{~S}$ in sulfate in per mil relative to Vienna Canyon Diablo Troilite; EF, East Fork of Many Devils Wash; GEMS, Geospatial Environmental Mapping System; -, not reported; n/a, not available]

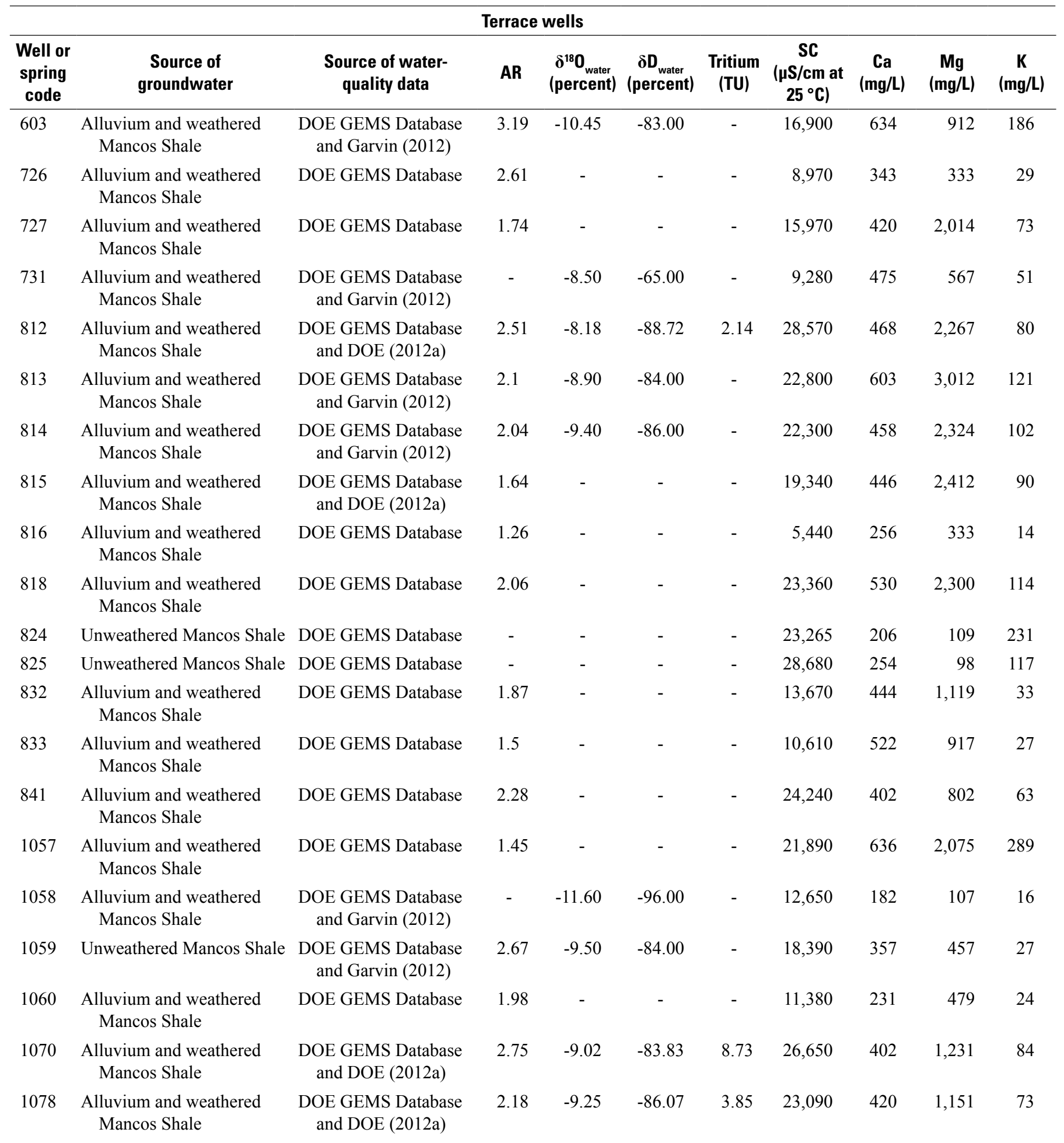


Table 1. Shiprock Disposal Site well information and selected sample data, Shiprock, New Mexico._Continued

$\left[\mathrm{AR},{ }^{234} \mathrm{U} /{ }^{238} \mathrm{U}\right.$ (uranium isotope) alpha activity ratio; $\delta^{18} \mathrm{O}$, isotopic composition of oxygen-18 in per mil relative to Vienna Standard Mean Ocean Water; $\delta \mathrm{D}$, isotopic composition of deuterium in per mil relative to Vienna Standard Mean Ocean Water; TU, tritium units; SC, specific conductivity; $\mu \mathrm{S} / \mathrm{cm}$ at $25^{\circ} \mathrm{C}$, microsiemens per centimeter at 25 degrees Celsius; Ca, calcium; mg/L, milligrams per liter; $\mathrm{Mg}$, magnesium; K, potassium; SCW, Salt Creek Wash; DOE, Department of Energy; <, less than; UENAS, Upper Eagle Nest Arroyo; $\mathrm{Na}$, sodium; $\mathrm{HCO}_{3}$, bicarbonate alkalinity as $\mathrm{CaCO}_{3} ; \mathrm{Cl}_{1} \mathrm{chloride}$, $\mathrm{SO}_{4}$, sulfate; $\mathrm{NO}_{3}$, nitrate as nitrate; $\mathrm{U}$, uranium; Se, selenium; $\mu \mathrm{g} / \mathrm{L}$, micrograms per liter; $\delta^{34} \mathrm{~S}_{\text {sulfate }}$, composition of ${ }^{34} \mathrm{~S}$ in sulfate in per mil relative to Vienna Canyon Diablo Troilite; EF, East Fork of Many Devils Wash; GEMS, Geospatial Environmental Mapping System; -, not reported; n/a, not available]

\begin{tabular}{|c|c|c|c|c|c|c|c|c|c|c|}
\hline \multicolumn{11}{|c|}{ Terrace wells } \\
\hline $\begin{array}{l}\text { Well or } \\
\text { spring } \\
\text { code }\end{array}$ & $\begin{array}{l}\text { Source of } \\
\text { groundwater }\end{array}$ & $\begin{array}{l}\text { Source of water- } \\
\text { quality data }\end{array}$ & $\underset{(\mathrm{mg} / \mathrm{L})}{\mathrm{Na}}$ & $\begin{array}{l}\mathrm{HCO}_{3} \\
(\mathrm{mg} / \mathrm{L})\end{array}$ & $\underset{(\mathrm{mg} / \mathrm{L})}{\mathrm{Cl}}$ & $\begin{array}{c}\mathrm{SO}_{4} \\
(\mathrm{mg} / \mathrm{L})\end{array}$ & $\begin{array}{c}\mathrm{NO}_{3} \\
(\mathrm{mg} / \mathrm{L})\end{array}$ & $\underset{(\mathrm{mg} / \mathrm{L})}{\mathbf{U}}$ & $\begin{array}{c}\text { Se } \\
(\mu \mathrm{g} / \mathrm{L})\end{array}$ & $\begin{array}{l}\delta^{34} S_{\text {sulfate }} \\
\text { (percent) }\end{array}$ \\
\hline 603 & $\begin{array}{l}\text { Alluvium and weathered } \\
\text { Mancos Shale }\end{array}$ & $\begin{array}{l}\text { DOE GEMS Database } \\
\text { and Garvin (2012) }\end{array}$ & 956 & 320 & 291 & 6,874 & 5,058 & 0.0112 & 196.1 & - \\
\hline 726 & $\begin{array}{l}\text { Alluvium and weathered } \\
\text { Mancos Shale }\end{array}$ & DOE GEMS Database & 1,833 & 588 & 243 & 5,887 & 122 & 0.0210 & 41.7 & - \\
\hline 727 & $\begin{array}{l}\text { Alluvium and weathered } \\
\text { Mancos Shale }\end{array}$ & DOE GEMS Database & 2,445 & 1,753 & 411 & 11,908 & 1,472 & 0.396 & 8.7 & - \\
\hline 731 & $\begin{array}{l}\text { Alluvium and weathered } \\
\text { Mancos Shale }\end{array}$ & $\begin{array}{l}\text { DOE GEMS Database } \\
\text { and Garvin (2012) }\end{array}$ & 1,107 & 405 & 317 & 4,714 & 1,092 & 0.039 & 160.9 & - \\
\hline 812 & $\begin{array}{l}\text { Alluvium and weathered } \\
\text { Mancos Shale }\end{array}$ & $\begin{array}{l}\text { DOE GEMS Database } \\
\text { and DOE (2012a) }\end{array}$ & 5,974 & 859 & 2,395 & 15,882 & 6,143 & 0.127 & 5,660 & -22.77 \\
\hline 815 & $\begin{array}{l}\text { Alluvium and weathered } \\
\text { Mancos Shale }\end{array}$ & $\begin{array}{l}\text { DOE GEMS Database } \\
\text { and DOE (2012a) }\end{array}$ & 3,189 & 1,715 & 689 & 14,619 & 2,969 & 0.306 & 157.6 & - \\
\hline 816 & $\begin{array}{l}\text { Alluvium and weathered } \\
\text { Mancos Shale }\end{array}$ & DOE GEMS Database & 713 & 432 & 122 & 2,978 & 249 & 0.0321 & 69.5 & - \\
\hline 818 & $\begin{array}{l}\text { Alluvium and weathered } \\
\text { Mancos Shale }\end{array}$ & DOE GEMS Database & 3,167 & 870 & 1,015 & 11,923 & 6,390 & 0.0925 & $2,286.3$ & - \\
\hline 824 & Unweathered Mancos Shale & DOE GEMS Database & 4,164 & 133 & 4,320 & 5,265 & 798 & 0.209 & 5.3 & - \\
\hline 825 & Unweathered Mancos Shale & DOE GEMS Database & 6,244 & 220 & 7,220 & 5,821 & 120 & 0.042 & 0.9 & - \\
\hline 1058 & $\begin{array}{l}\text { Alluvium and weathered } \\
\text { Mancos Shale }\end{array}$ & $\begin{array}{l}\text { DOE GEMS Database } \\
\text { and Garvin (2012) }\end{array}$ & 2,197 & 652 & 1,010 & 4,199 & 12 & 0.0057 & 0.6 & - \\
\hline 1059 & Unweathered Mancos Shale & $\begin{array}{l}\text { DOE GEMS Database } \\
\text { and Garvin (2012) }\end{array}$ & 3,626 & 801 & 674 & 8,871 & 1,553 & 0.0747 & 39.7 & - \\
\hline 1060 & $\begin{array}{l}\text { Alluvium and weathered } \\
\text { Mancos Shale }\end{array}$ & DOE GEMS Database & 2,056 & 607 & 290 & 5,908 & 1,129 & 0.187 & $1,911.5$ & - \\
\hline 1070 & $\begin{array}{l}\text { Alluvium and weathered } \\
\text { Mancos Shale }\end{array}$ & $\begin{array}{l}\text { DOE GEMS Database } \\
\text { and DOE (2012a) }\end{array}$ & 5,854 & 878 & 1,271 & 15,393 & 3,173 & 0.0829 & 2,730 & -21.71 \\
\hline 1078 & $\begin{array}{l}\text { Alluvium and weathered } \\
\text { Mancos Shale }\end{array}$ & $\begin{array}{l}\text { DOE GEMS Database } \\
\text { and DOE (2012a) }\end{array}$ & 5,019 & 730 & 1,109 & 13,785 & 3,001 & 0.129 & 2,730 & -22.20 \\
\hline
\end{tabular}


Table 1. Shiprock Disposal Site well information and selected sample data, Shiprock, New Mexico._Continued

$\left[\mathrm{AR},{ }^{234} \mathrm{U} /{ }^{238} \mathrm{U}\right.$ (uranium isotope) alpha activity ratio; $\delta^{18} \mathrm{O}$, isotopic composition of oxygen-18 in per mil relative to Vienna Standard Mean Ocean Water; $\delta \mathrm{D}$, isotopic composition of deuterium in per mil relative to Vienna Standard Mean Ocean Water; TU, tritium units; SC, specific conductivity; $\mu \mathrm{S} / \mathrm{cm}$ at $25^{\circ} \mathrm{C}$, microsiemens per centimeter at 25 degrees Celsius; $\mathrm{Ca}$, calcium; mg/L, milligrams per liter; $\mathrm{Mg}$, magnesium; K, potassium; SCW, Salt Creek Wash; DOE,

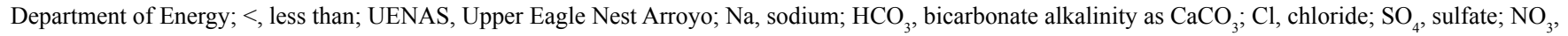
nitrate as nitrate; $\mathrm{U}$, uranium; Se, selenium; $\mu \mathrm{g} / \mathrm{L}$, micrograms per liter; $\delta^{34} \mathrm{~S}_{\text {sulfate }}$, composition of ${ }^{34} \mathrm{~S}$ in sulfate in per mil relative to Vienna Canyon Diablo Troilite; EF, East Fork of Many Devils Wash; GEMS, Geospatial Environmental Mapping System; -, not reported; n/a, not available]

\begin{tabular}{|c|c|c|c|c|c|c|c|c|c|c|}
\hline \multicolumn{11}{|c|}{ San Juan River } \\
\hline $\begin{array}{c}\text { Well or } \\
\text { spring code }\end{array}$ & $\begin{array}{c}\text { Source of } \\
\text { groundwater }\end{array}$ & $\begin{array}{l}\text { Source of water- } \\
\text { quality data }\end{array}$ & AR & $\begin{array}{c}\delta^{18} 0_{\text {water }} \\
\text { (percent) }\end{array}$ & $\begin{array}{c}\delta D_{\text {water }} \\
\text { (percent) }\end{array}$ & $\begin{array}{l}\text { Tritium } \\
\text { (TU) }\end{array}$ & $\begin{array}{c}\text { SC } \\
\left(\mu \mathrm{S} / \mathrm{cm} \text { at } 25^{\circ} \mathrm{C}\right)\end{array}$ & $\underset{(\mathrm{mg} / \mathrm{L})}{\mathrm{Ca}}$ & $\underset{(\mathrm{mg} / \mathrm{L})}{\mathrm{Mg}}$ & $\begin{array}{c}\mathrm{K} \\
(\mathrm{mg} / \mathrm{L})\end{array}$ \\
\hline $\begin{array}{c}\text { San Juan } \\
\text { River }\end{array}$ & $\mathrm{n} / \mathrm{a}$ & $\begin{array}{l}\text { DOE GEMS Database } \\
\text { and DOE }(2012 a)\end{array}$ & 1.31 & -12.10 & -90.89 & 6.39 & - & 62 & 35 & 4 \\
\hline
\end{tabular}

\begin{tabular}{|c|c|c|c|c|c|c|c|c|c|c|}
\hline \multicolumn{11}{|c|}{ San Juan River } \\
\hline $\begin{array}{c}\text { Well or } \\
\text { spring code }\end{array}$ & $\begin{array}{c}\text { Source of } \\
\text { groundwater }\end{array}$ & $\begin{array}{l}\text { Source of water- } \\
\text { quality data }\end{array}$ & $\underset{(\mathrm{mg} / \mathrm{L})}{\mathrm{Na}}$ & $\begin{array}{l}\mathrm{HCO}_{3} \\
\text { (mg/L) }\end{array}$ & $\underset{(\mathrm{mg} / \mathrm{L})}{\mathrm{CI}}$ & $\underset{(\mathrm{mg} / \mathrm{L})}{\mathrm{SO}_{4}}$ & $\begin{array}{c}\mathrm{NO}_{3} \\
(\mathrm{mg} / \mathrm{L})\end{array}$ & $\underset{\text { (mg/L) }}{U}$ & $\begin{array}{c}\mathrm{Se} \\
(\mu \mathrm{g} / \mathrm{L})\end{array}$ & $\begin{array}{c}\delta^{34} S_{\text {sulfate }} \\
\text { (percent) }\end{array}$ \\
\hline $\begin{array}{c}\text { San Juan } \\
\text { River }\end{array}$ & $\mathrm{n} / \mathrm{a}$ & $\begin{array}{l}\text { DOE GEMS Database } \\
\text { and DOE (2012a) }\end{array}$ & 46 & 116 & 14 & 130 & - & $<0.0017$ & $<10$ & 1.07 \\
\hline
\end{tabular}

\begin{tabular}{|c|c|c|c|c|c|c|c|c|c|c|}
\hline \multicolumn{11}{|c|}{ Morrison Formation } \\
\hline $\begin{array}{c}\text { Well or } \\
\text { spring code }\end{array}$ & $\begin{array}{c}\text { Source of } \\
\text { groundwater }\end{array}$ & $\begin{array}{l}\text { Source of water- } \\
\text { quality data }\end{array}$ & AR & $\begin{array}{c}\delta^{18} 0_{\text {water }} \\
\text { (percent) }\end{array}$ & $\begin{array}{c}\delta D_{\text {water }} \\
\text { (percent) }\end{array}$ & $\begin{array}{l}\text { Tritium } \\
\text { (TU) }\end{array}$ & $\begin{array}{c}\text { SC } \\
\left(\mu \mathrm{S} / \mathrm{cm} \text { at } 25^{\circ} \mathrm{C}\right)\end{array}$ & $\begin{array}{c}\mathrm{Ca} \\
(\mathrm{mg} / \mathrm{L})\end{array}$ & $\underset{(\mathrm{mg} / \mathrm{L})}{\mathrm{Mg}}$ & $\begin{array}{c}\mathrm{K} \\
(\mathrm{mg} / \mathrm{L})\end{array}$ \\
\hline
\end{tabular}

\begin{tabular}{|c|c|c|c|c|c|c|c|c|c|c|}
\hline \multicolumn{11}{|c|}{ Morrison Formation } \\
\hline $\begin{array}{c}\text { Well or } \\
\text { spring code }\end{array}$ & $\begin{array}{c}\text { Source of } \\
\text { groundwater }\end{array}$ & $\begin{array}{c}\text { Source of water- } \\
\text { quality data }\end{array}$ & $\begin{array}{c}\mathrm{Na} \\
(\mathrm{mg} / \mathrm{L})\end{array}$ & $\begin{array}{l}\mathrm{HCO}_{3} \\
(\mathrm{mg} / \mathrm{L})\end{array}$ & $\begin{array}{c}\mathrm{Cl} \\
(\mathrm{mg} / \mathrm{L})\end{array}$ & $\begin{array}{c}\mathrm{SO}_{4} \\
(\mathrm{mg} / \mathrm{L})\end{array}$ & $\begin{array}{c}\mathrm{NO}_{3} \\
(\mathrm{mg} / \mathrm{L})\end{array}$ & $\begin{array}{c}\mathbf{U} \\
(\mathrm{mg} / \mathrm{L})\end{array}$ & $\underset{(\mu \mathrm{g} / \mathrm{L})}{\mathrm{Se}}$ & $\begin{array}{c}\delta^{34} S_{\text {sulfate }} \\
\text { (percent) }\end{array}$ \\
\hline 648 & $\begin{array}{l}\text { Morrison } \\
\text { Formation }\end{array}$ & $\begin{array}{l}\text { DOE GEMS Database } \\
\text { and Dam (1995) }\end{array}$ & 817 & 64.4 & 52.5 & 1,974 & $<0.10$ & - & $<1$ & 10.2 \\
\hline
\end{tabular}

\begin{tabular}{|c|c|c|c|c|c|c|c|c|c|c|}
\hline \multicolumn{11}{|c|}{ Gallup Formation } \\
\hline $\begin{array}{c}\text { Well or } \\
\text { spring code }\end{array}$ & $\begin{array}{c}\text { Source of } \\
\text { groundwater }\end{array}$ & $\begin{array}{l}\text { Source of water- } \\
\text { quality data }\end{array}$ & AR & $\begin{array}{l}\delta^{18} 0_{\text {water }} \\
\text { (percent) }\end{array}$ & $\begin{array}{c}\delta D_{\text {water }} \\
\text { (percent) }\end{array}$ & $\begin{array}{l}\text { Tritium } \\
\text { (TU) }\end{array}$ & $\begin{array}{c}\text { SC } \\
\left(\mu \mathrm{S} / \mathrm{cm} \text { at } 25^{\circ} \mathrm{C}\right)\end{array}$ & $\begin{array}{c}\mathrm{Ca} \\
(\mathrm{mg} / \mathrm{L})\end{array}$ & $\underset{(\mathrm{mg} / \mathrm{L})}{\mathrm{Mg}}$ & $\begin{array}{c}\mathrm{K} \\
(\mathrm{mg} / \mathrm{L})\end{array}$ \\
\hline
\end{tabular}

\begin{tabular}{lll} 
spring code & groundwater & quality data \\
\hline Gallup & Gallup & Dam (1995) \\
& Sandstone & \\
& &
\end{tabular}

\begin{tabular}{|c|c|c|c|c|c|c|c|c|c|c|}
\hline \multicolumn{11}{|c|}{ Gallup Formation } \\
\hline $\begin{array}{c}\text { Well or } \\
\text { spring code }\end{array}$ & $\begin{array}{c}\text { Source of } \\
\text { groundwater }\end{array}$ & $\begin{array}{c}\text { Source of water- } \\
\text { quality data }\end{array}$ & $\underset{(\mathrm{mg} / \mathrm{L})}{\mathrm{Na}}$ & $\begin{array}{l}\mathrm{HCO}_{3} \\
(\mathrm{mg} / \mathrm{L})\end{array}$ & $\begin{array}{c}\mathrm{Cl} \\
(\mathrm{mg} / \mathrm{L})\end{array}$ & $\begin{array}{c}\mathrm{SO}_{4} \\
(\mathrm{mg} / \mathrm{L})\end{array}$ & $\begin{array}{c}\mathrm{NO}_{3} \\
(\mathrm{mg} / \mathrm{L})\end{array}$ & $\begin{array}{c}\mathbf{U} \\
(\mathrm{mg} / \mathrm{L})\end{array}$ & $\begin{array}{c}\mathrm{Se} \\
(\mu \mathrm{g} / \mathrm{L})\end{array}$ & $\begin{array}{c}\delta^{34} S_{\text {sulfate }} \\
\text { (percent) }\end{array}$ \\
\hline Gallup & $\begin{array}{l}\text { Gallup } \\
\text { Sandstone }\end{array}$ & Dam (1995) & - & - & - & - & - & - & - & -14.0 to 15.4 \\
\hline
\end{tabular}

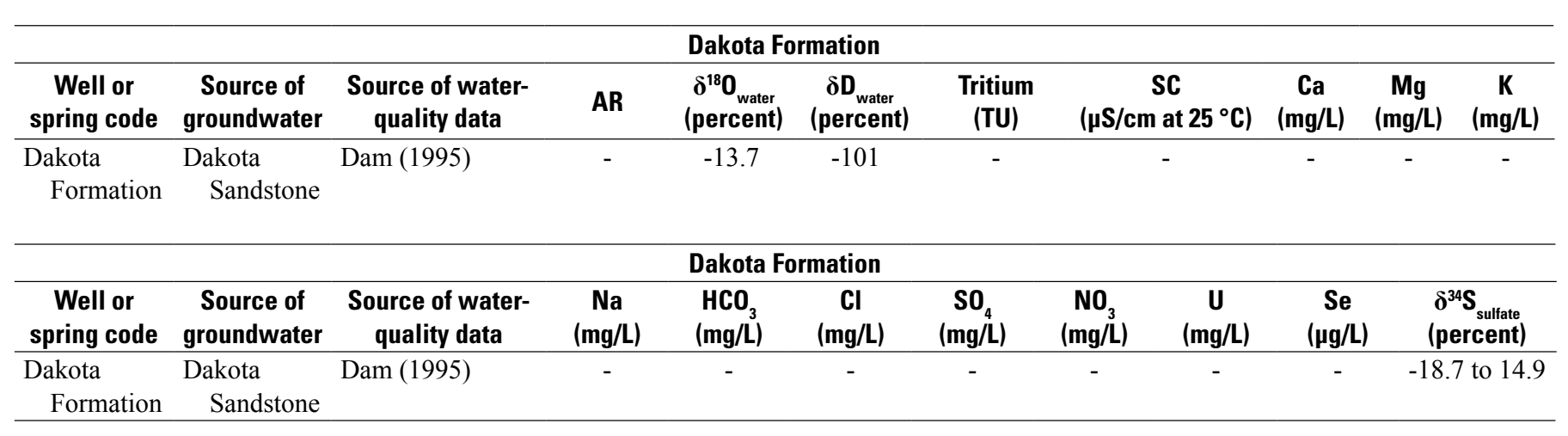


Additional groundwater samples were collected from selected wells in October and November 2012 by the USGS following USGS procedures (U.S. Geological Survey, variously dated). Groundwater was collected from wells at various locations around the disposal cell and in Many Devils Wash (fig. 4). Wells were selected on the basis of their location relative to the disposal cell and on their capacity to produce enough groundwater to collect samples. Samples were analyzed for metals, inorganic anions, dissolved solids, alkalinity, groundwater-age indicators, and dissolved gases. The samples were collected by using dedicated pumps, which included low-flow bladder pumps (wells 603, 728, 731, 813, 815, 817, $824,827,833$, and 841 ) operated by using compressed nitrogen gas and peristaltic pumps with dedicated tubing (well 1048). Prior to sample collection, measurements of $\mathrm{pH}$, temperature, specific conductance (SC), and dissolved oxygen (DO) were made until parameters were stable (table 2). Water levels were also measured periodically during sampling to track drawdown and to ensure that the well did not go dry. Groundwater samples were filtered through 0.45 -micrometer $(\mu \mathrm{m})$ filters at most wells for cation and anion (including alkalinity) analysis. Wells 728 and 815 were sampled without the filter because of an oversight and are reported as total rather than dissolved concentrations. Metals, inorganic anions, dissolved solids, and alkalinity were analyzed using EPA methods SW846 6010B, MCAWW 300, SM 2540C, and SM 2320B, respectively, by the USGS contract laboratory TestAmerica in Arvada, Colo. No blanks were collected because of the use of dedicated equipment. Data are stored in the USGS National Water Information System (http:// dx.doi.org/10.5066/F7P55KJN).

\section{Apparent Groundwater-Age Analysis}

Chemical and isotopic constituents that have been released into the atmosphere at unique rates and interact with atmospheric water may be introduced to the groundwater and can be used to estimate the apparent age of groundwater (Plummer and Friedman, 1999). Four age-dating tracers were collected from various wells to determine the apparent age of the groundwater at the Shiprock Disposal Site and in Many Devils Wash.

\section{Tritium}

Tritium $\left({ }^{3} \mathrm{H}\right)$ is a radioactive isotope of hydrogen that is short-lived with a half-life of 12.32 years (Lucas and Unterweger, 2000). During the late 1950s to the mid1960 s, testing of nuclear weapons raised the atmospheric concentrations of ${ }^{3} \mathrm{H}$ hundreds of times above the normal background concentrations (Plummer and Friedman, 1999). In 1963, the Nuclear Test Ban Treaty was signed, eliminating most of the aboveground nuclear weapons testing in the world. Since then, ${ }^{3} \mathrm{H}$ levels in the atmosphere have decreased to natural levels. These ${ }^{3} \mathrm{H}$ concentrations from nuclear weapons testing continue to be present in some groundwater and may be used to qualitatively constrain the recharge date (Clark and Fritz, 1997). In the atmosphere, ${ }^{3} \mathrm{H}$ is rapidly oxidized and enters the hydrologic cycle, making it a valuable tracer for timescales less than 100 years. One significant advantage to using ${ }^{3} \mathrm{H}$ is that it is incorporated into the water molecule and is not affected by chemical reactions, microbial degradation, or other reactions that complicate the interpretation of other tracers. In addition, the ${ }^{3} \mathrm{H}$ concentration in precipitation is not influenced by temperature (as are oxygen-18 $\left[{ }^{18} \mathrm{O}\right]$ and deuterium [D]), and the only fractionation (the enrichment of one isotope relative to another in the reactant or product of a chemical or physical process) is caused by evaporation following precipitation, where ${ }^{3} \mathrm{H}$ is fractionated at about twice the extent of $\mathrm{D}$ (Bolin, 1958). Evaporative fractionation of ${ }^{3} \mathrm{H}$ would affect the apparent age differently depending on whether the age was determined to be before or after the peak atmospheric concentrations in 1963. If the apparent age is interpreted as after 1963, the enrichment of ${ }^{3} \mathrm{H}$ through evaporation would bias the apparent age older than the actual time when the water infiltrated. If the apparent age is interpreted as before 1963, the age would be biased younger than the actual age.

Tritium samples were collected in two 500-milliliter $(\mathrm{mL})$, high-density polyethylene bottles and sealed with polycone caps. The samples were collected by using a dedicated low-flow bladder or peristaltic pump. Samples were collected from nine wells around the Shiprock Disposal Site and one sample from a well (1048) within Many Devils Wash (table 2). Samples were collected in replicate in case of sample loss, but only one sample from each site was submitted for analysis to the Noble Gas Laboratory, Lamont-Doherty Earth Observatory, Columbia University, New York. The ${ }^{3} \mathrm{H}$ activity was determined by helium ingrowth (Clarke and others, 1976; Bayer and others, 1989). Because the decay rate is known, the ${ }^{3} \mathrm{H}$ activity can be determined. Tritium activity is reported in ${ }^{3} \mathrm{H}$ units (TU) where $1 \mathrm{TU}$ is defined as 1 atom of ${ }^{3} \mathrm{H}$ in $10^{18}$ atoms of hydrogen.

\section{Helium-3 Samples}

Radioactive decay of ${ }^{3} \mathrm{H}$ produces the noble gas helium-3 $\left({ }^{3} \mathrm{He}\right)$ (The Reston Groundwater Dating Laboratory, 2014). By measuring the ${ }^{3} \mathrm{H}$ and ${ }^{3} \mathrm{He}$ concentration from a groundwater sample, an apparent age can be estimated.

Helium samples were collected from many of the same wells as ${ }^{3} \mathrm{H}$ (table 2). The helium (along with neon) samples were collected by using special pinch-off copper tubes (3/8in. diameter, 30-in. length emplaced in an aluminum sleeve with stainless-steel pinch-off clamps). The copper tubes were flushed with sample water and then sealed after determining that no air bubbles were present in the copper tube. Samples were collected in duplicate and sent to the Noble Gas Laboratory, Lamont-Doherty Earth Observatory for helium (and neon) analyses and determination of the ${ }^{3} \mathrm{He} /{ }^{4} \mathrm{He}$ isotope ratio of dissolved helium; however, low neon and helium concentrations in all the samples suggested gas loss from groundwater and prevented their use in constraining the ${ }^{3} \mathrm{H}$ date. The gases may have been stripped from the groundwater because of the high carbon dioxide $\left(\mathrm{CO}_{2}\right)$ present in the water (Robert Newton, Lamont-Doherty Earth Observatory, written commun., 2013). 
Table 2. Field parameters and analytical results from U.S. Geological Survey sampling event in October and November 2012.

[ID, identifier; TU, tritium units; $\mathrm{N}_{2}$, nitrogen; mg/L, milligrams per liter; $\mathrm{Ar}$, argon; $\mathrm{O}_{2}$, oxygen; $\mathrm{CO}_{2}$, carbon dioxide; $\mathrm{CH}_{4}$, methane; ${ }^{4} \mathrm{He}$, helium-4; ccSTP/g, cubic centimeters of gas at standard temperature ( 0 degrees Celsius), and pressure (1 atmosphere) per gram of water; Ne, neon; $\mathrm{CFC}$, chlorofluorocarbons; pptv, parts per trillion volume; ${ }^{13} \mathrm{C}$, carbon-13 in per mil relative to Vienna Pee Dee Belemnite (VPDB); ${ }^{14} \mathrm{C}$, carbon-14 in denormalized percent modern carbon (pmc); $\mathrm{pH}$, the negative $\log$ of the activity of the hydrogen ion; $\mathrm{AlK} / \mathrm{ANC}$, alkalinity (or acid neutralizing capacity) as calcium carbonate $\left(\mathrm{CaCO}_{3}\right)$; $\mathrm{T}$, temperature; $\mathrm{SC}$, specific conductivity; $\mu \mathrm{S} / \mathrm{cm}$ at $25 \mathrm{C}^{\circ}$, microsiemens per centimeter at 25 degrees Celsius; DO, dissolved oxygen; DS, dissolved solids; Ca, calcium; Mg, magnesium; K, potassium; $\mathrm{Na}$, sodium; $\mathrm{HCO}_{3}$, bicarbonate alkalinity as $\mathrm{CaCO}_{3} ; \mathrm{Cl}$, chloride; $\mathrm{SO}_{4}$, sulfate; $\mathrm{NO}_{3}$, nitrate; *, major ions reported as total and acid neutralizing capacity; -, not reported; PI, partial pressure]

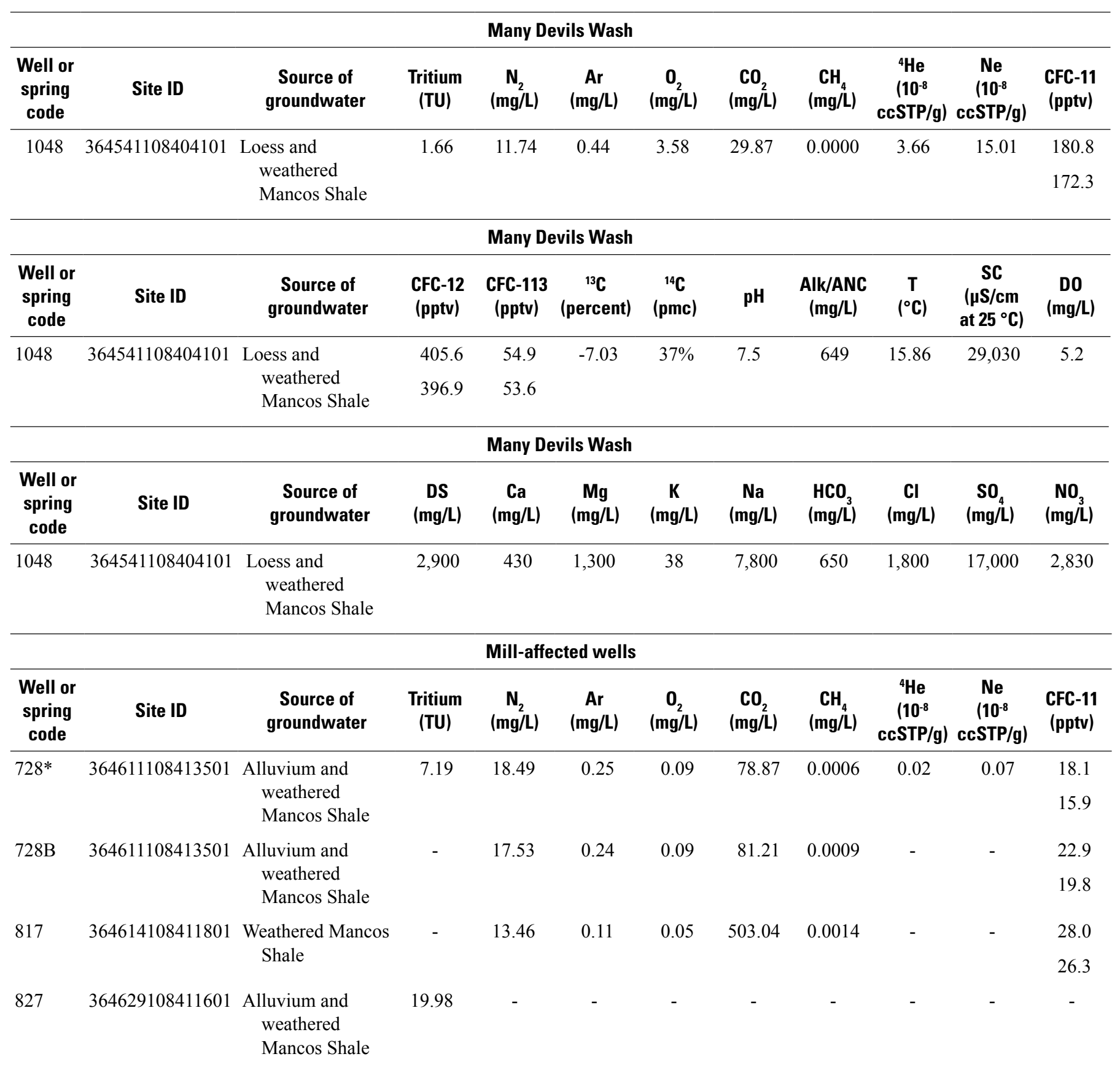


Table 2. Field parameters and analytical results from U.S. Geological Survey sampling event in 0ctober and November 2012.Continued

[ID, identifier; TU, tritium units; $\mathrm{N}_{2}$, nitrogen; mg/L, milligrams per liter; Ar, argon; $\mathrm{O}_{2}$, oxygen; $\mathrm{CO}_{2}$, carbon dioxide; $\mathrm{CH}_{4}$, methane; ${ }^{4} \mathrm{He}$, helium-4; ccSTP/g, cubic centimeters of gas at standard temperature ( 0 degrees Celsius), and pressure (1 atmosphere) per gram of water; Ne, neon; CFC, chlorofluorocarbons; pptv, parts per trillion volume; ${ }^{13} \mathrm{C}$, carbon-13 in per mil relative to Vienna Pee Dee Belemnite (VPDB); ${ }^{14} \mathrm{C}$, carbon-14 in denormalized percent modern carbon (pmc); $\mathrm{pH}$, the negative log of the activity of the hydrogen ion; $\mathrm{AlK} / \mathrm{ANC}$, alkalinity (or acid neutralizing capacity) as calcium carbonate $\left(\mathrm{CaCO}_{3}\right)$; $\mathrm{T}$, temperature; $\mathrm{SC}$, specific conductivity; $\mu \mathrm{S} / \mathrm{cm}$ at $25 \mathrm{C}^{\circ}$, microsiemens per centimeter at 25 degrees Celsius; DO, dissolved oxygen; DS, dissolved solids; Ca, calcium; Mg, magnesium; K, potassium; $\mathrm{Na}$, sodium; $\mathrm{HCO}_{3}$, bicarbonate alkalinity as $\mathrm{CaCO}_{3} ; \mathrm{Cl}$, chloride; $\mathrm{SO}_{4}$, sulfate; $\mathrm{NO}_{3}$, nitrate; *, major ions reported as total and acid neutralizing capacity; -, not reported; PI, partial pressure]

\begin{tabular}{|c|c|c|c|c|c|c|c|c|c|c|c|}
\hline \multicolumn{12}{|c|}{ Mill-affected wells } \\
\hline $\begin{array}{c}\text { Well or } \\
\text { spring } \\
\text { code }\end{array}$ & Site ID & $\begin{array}{l}\text { Source of } \\
\text { groundwater }\end{array}$ & $\begin{array}{l}\text { CFC-12 } \\
\text { (pptv) }\end{array}$ & $\begin{array}{l}\text { CFC-113 } \\
\text { (pptv) }\end{array}$ & $\begin{array}{c}{ }^{13} \mathrm{C} \\
\text { (percent) } \\
\text { VPDB }\end{array}$ & $\begin{array}{c}{ }^{14} \mathrm{C} \\
(\mathrm{pmc})\end{array}$ & pH & $\begin{array}{c}\text { Alk/ANC } \\
\text { (mg/L as } \\
\mathrm{CaCO}_{3} \text { ) }\end{array}$ & $\begin{array}{c}\mathrm{T} \\
\left({ }^{\circ} \mathrm{C}\right)\end{array}$ & $\begin{array}{c}S C \\
(\mu S / c m \\
\left.\text { at } 25^{\circ} \mathrm{C}\right)\end{array}$ & $\begin{array}{c}\mathrm{DO} \\
(\mathrm{mg} / \mathrm{L})\end{array}$ \\
\hline \multirow[t]{2}{*}{$728 *$} & \multirow[t]{2}{*}{364611108413501} & \multirow{2}{*}{$\begin{array}{l}\text { Alluvium and weathered } \\
\text { Mancos Shale }\end{array}$} & 112.1 & 6.2 & \multirow[t]{2}{*}{-4.62} & \multirow[t]{2}{*}{14} & \multirow[t]{2}{*}{7} & \multirow[t]{2}{*}{290} & \multirow[t]{2}{*}{14.6} & \multirow[t]{2}{*}{5,270} & \multirow[t]{2}{*}{0.6} \\
\hline & & & 108.6 & 5.6 & & & & & & & \\
\hline $728 \mathrm{~B}$ & 364611108413501 & $\begin{array}{l}\text { Alluvium and weathered } \\
\text { Mancos Shale }\end{array}$ & 127.9 & 7.8 & - & - & - & - & - & - & - \\
\hline \multirow[t]{2}{*}{817} & \multirow[t]{2}{*}{364614108411801} & \multirow{2}{*}{$\begin{array}{l}\text { Weathered Mancos } \\
\text { Shale }\end{array}$} & 163.7 & 11.0 & \multirow[t]{2}{*}{-} & \multirow[t]{2}{*}{-} & \multirow[t]{2}{*}{6.9} & \multirow[t]{2}{*}{-} & \multirow[t]{2}{*}{15.8} & \multirow[t]{2}{*}{18,500} & \multirow[t]{2}{*}{0.6} \\
\hline & & & 175.2 & 11.1 & & & & & & & \\
\hline 827 & 364629108411601 & $\begin{array}{l}\text { Alluvium and weathered } \\
\text { Mancos Shale }\end{array}$ & - & - & -6.55 & 6 & 7 & 1,700 & 16.9 & 10,270 & 0.2 \\
\hline
\end{tabular}



\begin{tabular}{|c|c|c|c|c|c|c|c|c|c|c|c|}
\hline \multicolumn{12}{|c|}{ Terrace wells } \\
\hline $\begin{array}{c}\text { Well or } \\
\text { spring } \\
\text { code }\end{array}$ & Site ID & $\begin{array}{l}\text { Source of } \\
\text { groundwater }\end{array}$ & $\begin{array}{l}\text { Tritium } \\
\text { (TU) }\end{array}$ & $\begin{array}{c}\mathrm{N}_{2} \\
(\mathrm{mg} / \mathrm{L})\end{array}$ & $\underset{(\mathrm{mg} / \mathrm{L})}{\mathrm{Ar}}$ & $\begin{array}{c}0_{2} \\
(\mathrm{mg} / \mathrm{L})\end{array}$ & $\begin{array}{c}\mathrm{CO}_{2} \\
(\mathrm{mg} / \mathrm{L})\end{array}$ & $\begin{array}{c}\text { CH4 } \\
\text { (mg/L) }\end{array}$ & $\begin{array}{c}{ }^{4} \mathrm{He} \\
\left(10^{-8}\right. \\
\text { ccSTP/g) }\end{array}$ & $\begin{array}{c}\mathrm{Ne} \\
\left(10^{-8}\right. \\
\text { ccSTP/g) }\end{array}$ & $\begin{array}{l}\text { CFC-11 } \\
\text { (pptv) }\end{array}$ \\
\hline 603 & 364553108410001 & $\begin{array}{l}\text { Alluvium and weathered } \\
\text { Mancos Shale }\end{array}$ & 12.98 & - & - & - & - & - & 2.16 & 9.52 & - \\
\hline 731 & 364548108405701 & $\begin{array}{l}\text { Alluvium and weathered } \\
\text { Mancos Shale }\end{array}$ & 6.12 & - & - & - & - & - & 5.55 & 6.96 & - \\
\hline $815^{*}$ & 364621108414701 & $\begin{array}{l}\text { Alluvium and weathered } \\
\text { Mancos Shale }\end{array}$ & 33.21 & 12.12 & 0.09 & 0.6 & 385.55 & 0.0003 & 0.29 & 0.86 & $\begin{array}{l}4.8 \\
0.0\end{array}$ \\
\hline $815 B$ & 364621108414701 & $\begin{array}{l}\text { Alluvium and weathered } \\
\text { Mancos Shale }\end{array}$ & - & 12.77 & 0.02 & 0.0 & 428.78 & 0.0003 & - & - & $\begin{array}{l}16.8 \\
12.0\end{array}$ \\
\hline 833 & 364632108420901 & $\begin{array}{l}\text { Alluvium and weathered } \\
\text { Mancos Shale }\end{array}$ & 5.54 & 14.60 & 0.43 & 1.0 & 59.80 & 0.0000 & 3.02 & 13.19 & $\begin{array}{l}125.5 \\
126.6\end{array}$ \\
\hline 841 & 364604108420401 & $\begin{array}{l}\text { Alluvium and weathered } \\
\text { Mancos Shale }\end{array}$ & 3.43 & 16.16 & 0.36 & 0.1 & 69.59 & 0.0002 & - & - & $\begin{array}{l}123.0 \\
121.7\end{array}$ \\
\hline
\end{tabular}


Table 2. Field parameters and analytical results from U.S. Geological Survey sampling event in October and November 2012.Continued

[ID, identifier; TU, tritium units; $\mathrm{N}_{2}$, nitrogen; mg/L, milligrams per liter; Ar, argon; $\mathrm{O}_{2}$, oxygen; $\mathrm{CO}_{2}$, carbon dioxide; $\mathrm{CH}_{4}$, methane; ${ }^{4} \mathrm{He}$, helium-4; ccSTP/g, cubic centimeters of gas at standard temperature (0 degrees Celsius), and pressure ( 1 atmosphere) per gram of water; Ne, neon; $\mathrm{CFC}$, chlorofluorocarbons; pptv, parts per trillion volume; ${ }^{13} \mathrm{C}$, carbon-13 in per mil relative to Vienna Pee Dee Belemnite (VPDB); ${ }^{14} \mathrm{C}$, carbon-14 in denormalized percent modern carbon (pmc); $\mathrm{pH}$, the negative $\log$ of the activity of the hydrogen ion; AlK/ANC, alkalinity (or acid neutralizing capacity) as calcium carbonate $\left(\mathrm{CaCO}_{3}\right) ; \mathrm{T}$, temperature; $\mathrm{SC}$, specific conductivity; $\mu \mathrm{S} / \mathrm{cm}$ at $25 \mathrm{C}^{\circ}$, microsiemens per centimeter at 25 degrees Celsius; DO, dissolved oxygen; DS, dissolved solids; Ca, calcium; $\mathrm{Mg}$, magnesium; K, potassium; $\mathrm{Na}$, sodium; $\mathrm{HCO}_{3}$, bicarbonate alkalinity as $\mathrm{CaCO}_{3} ; \mathrm{Cl}$, chloride; $\mathrm{SO}_{4}$, sulfate; $\mathrm{NO}_{3}$, nitrate; , major ions reported as total and acid neutralizing capacity; -, not reported; PI, partial pressure]

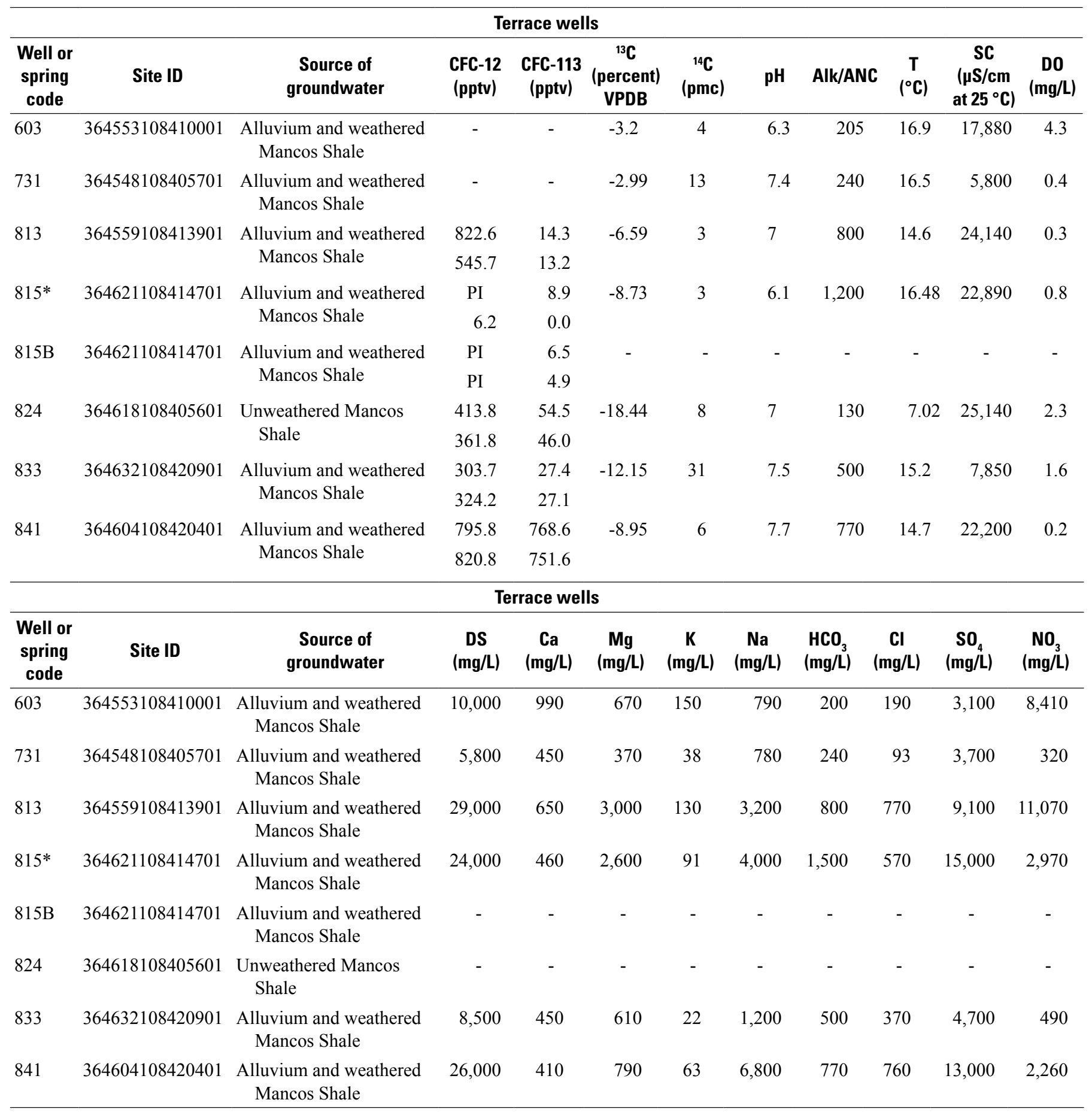




\section{Carbon-13 and Carbon-14}

The dissolved inorganic carbon samples for carbon-13 $\left({ }^{13} \mathrm{C}\right)$ and carbon-14 $\left({ }^{14} \mathrm{C}\right)$ to carbon-12 $\left({ }^{12} \mathrm{C}\right)$ analysis were collected by using a dedicated low-flow bladder or peristaltic pump from wells with dedicated tubing. The filtered samples were collected in two 1,000-mL safety-coated glass bottles and sealed with polycone caps. Samples were collected from nine Terrace and Mill-affected wells and one sample was collected from a well in Many Devils Wash (table 2). Samples were submitted to the National Ocean Sciences Accelerator Mass Spectrometry Facility, the Woods Hole Oceanographic Institution, Woods Hole, Massachusetts, and analyzed by mass spectroscopy (National Ocean Sciences Accelerator Mass Spectrometry Facility, 2015).

The stable carbon isotope composition, delta ${ }^{13} \mathrm{C}\left(\delta^{13} \mathrm{C}\right)$, is a useful indicator of the dissolved inorganic carbon sources in groundwater (Chapelle and LaRoy, 1985). This is because the different isotopic signatures of $\mathrm{CO}_{2}$, calcite and other organic constituents that may become part of the dissolved phase in groundwater (for a discussion of the observed ranges of $\delta^{13} \mathrm{C}$, see Wang and others, 1998), and $\delta^{13} \mathrm{C}$ is a useful indicator of the inorganic carbon (for a discussion of the observed ranges of $\delta^{13} \mathrm{C}$, see Wang and others, 1998).

Carbon-14 has a half-life of 5,730 years and is produced in the upper atmosphere by the interaction of cosmic rays and atmospheric nitrogen. The cosmogenic ${ }^{14} \mathrm{C}$ is rapidly oxidized and becomes incorporated into the biologic and hydrologic processes as carbon dioxide $\left({ }^{14} \mathrm{CO}_{2}\right)$ (Plummer and others, 2004). Other processes that may contribute inorganic ${ }^{14} \mathrm{C}$ to groundwater include carbonate mineral dissolution, coalification, methane production, metamorphism of carbonate rocks, and anaerobic biochemical decomposition of organic material (Wigley and others, 1978; Phillips and others, 1989). The interpretation of ${ }^{14} \mathrm{C}$ dates is complicated when these contributors are present because of the difficulty in determining the amount of ${ }^{14} \mathrm{C}$ that has been added to the system by each source. On the basis of the measurable methane detected in the samples and the evidence of reducing conditions, as well as the high $\mathrm{CO}_{2}$ concentrations in the groundwater, the ${ }^{14} \mathrm{C}$ was not considered a reliable indicator of age and is therefore not interpreted in the investigation. This decision is supported by the large range of ${ }^{13} \mathrm{C}$ values $(-18.44$ to -2.99 ) measured in the groundwater (table 2).

\section{Chlorofluorocarbons}

The production of chlorofluorocarbons (CFCs) began with CFC-12 in 1931, followed by CFC-11 in 1936, and then by many other CFC compounds, most notably CFC-113. CFC-12 and CFC-11 were primarily used as coolants, blowing agents, propellants, and solvents (Plummer and Friedman, 1999). CFC-113 was used by the electronics industry in semiconductor chip manufacturing in vapor degreasing and cold immersion cleaning of microelectronic parts and surface cleaning (Plummer and Friedman, 1999). CFC-12 was the first produced and its presence in groundwater indicates recharge occurred after 1940. The presence of CFC-11 indicates that recharge occurred after 1945, and the presence of CFC113 indicates that recharge occurred after 1965 (Bartolino, 1997). The "Montreal Protocol on Substances that Deplete the Ozone Layer" was established to stop CFC production in industrialized countries in 1996 (Plummer and Friedman, 1999). Since then, CFC concentrations in the atmosphere have leveled off or slightly declined (Plummer and Friedman, 1999).

The age of groundwater is estimated from CFC data by comparing concentrations of CFCs in groundwater to the historical atmospheric concentrations of CFCs. As with most chemical tracers, biochemical processes can influence the concentrations of CFCs in groundwater. For instance, CFCs, particularly CFC-11, may be lost because of microbial degradation, thus leading to an older estimate of age. Other assumptions and factors that can affect the interpreted age include the temperature of the water table during recharge, the thickness of the unsaturated zone, the entrapment of excess air, uncertainty of recharge elevation, and the mixing of younger and older water in the aquifer (Plummer and Friedman, 1999). Introduction of atmospheric air during sampling will produce a younger $\mathrm{CFC}$ model date.

The samples for CFCs analysis were collected in $125-\mathrm{mL}$ clear bottles and sealed with an aluminum-lined cap. Samples were collected by using a dedicated low-flow bladder or peristaltic pump from wells with dedicated tubing. To prevent contamination from the atmosphere, the sample bottles were placed into a 2-liter (L) plastic beaker, groundwater pumped from the well was allowed to fill and overflow the sample bottle and the beaker, and the sample bottle was capped while submerged. Replicate samples were shipped to the USGS CFC laboratory in Reston, Virginia, for analysis by purge-and-trap gas chromatography with an electron-capture detector (http:// water.usgs.gov/lab/chlorofluorocarbons). The concentrations of CFCs are reported in parts per trillion volume (pptv). According to Cook and others (2006), new sample tubing may be a source of CFC contamination. Because the tubing on the dedicated equipment is more than 3 years old and constructed of polyethylene, the risk of contamination was regarded to be fairly low. To ensure this was the case, additional replicate CFC samples were collected from two wells by using a Waterra Hydrolift-2 pump and nylon tubing. Limited water, depths to groundwater, and access restricted the use of this technique to all but a few wells.

\section{Dissolved Gases}

Argon (Ar) and nitrogen $\left(\mathrm{N}_{2}\right)$ concentrations are measured in groundwater to estimate the temperature and excess air of the groundwater recharging the aquifer. This is a useful method to help determine the concentrations of CFCs in the aquifer at the time of recharge (Aeschbach-Hertig and others, 1999).

The dissolved gases (methane, $\mathrm{CO}_{2}, \mathrm{~N}_{2}$, oxygen $\left[\mathrm{O}_{2}\right]$, and Ar) were collected using a dedicated low-flow bladder or peristaltic pump. A 125-mL clear Boston round bottle 
was placed into a 2-L plastic beaker. Groundwater pumped from the well was allowed to fill and overflow the sample bottle and the beaker. This sampling process allows for the collection of groundwater samples without interaction between groundwater and atmosphere. The bottle was sealed with a rubber stopper and pierced by a syringe needle while the bottle was still submerged. The syringe needle was then removed from the stopper while underwater to ensure that no air bubbles were present in the bottle. The concentrations of dissolved gases were determined by gas chromatography at the USGS Dissolved Gas Laboratory in Reston, Va. (The Reston Groundwater Dating Laboratory, 2015a).

Extremely low nitrogen and argon concentrations in all the samples suggested a gas loss and prevented their use in determining the temperature of the recharge water and the amount of excess air; therefore, the recharge conditions of the CFCs in groundwater had to be estimated. The average annual temperature (Western Regional Climate Center, 2012) for Shiprock $\left(55.3^{\circ} \mathrm{F}\right)$ was assumed to be the recharge temperature, and the elevation was assumed to be $4,980 \mathrm{ft}$ above the North American Vertical Datum of 1988 (NAVD 88). As with neon and helium, the gas loss from groundwater may have occurred because of the high $\mathrm{CO}_{2}$ present in the samples (Peggy Widman, U.S. Geological Survey, Reston Groundwater Dating Laboratory, written commun., 2013). Based on a sensitivity analysis, an error in the estimated recharge temperature of $18^{\circ} \mathrm{F}$ can result in a model date error of approximately 5 years in most circumstances. The sensitivity analysis was performed using the USGS spreadsheet program for preliminary evaluation of CFC data (The Reston Groundwater Dating Laboratory, 2015b).

\section{X-Ray Diffraction}

Rock samples were analyzed by the USGS X-Ray Diffraction Laboratory in Denver, Colo. Each sample was evaluated for zones of inhomogeneity, and subsamples, if present, were taken from these zones. Samples were lightly crushed and passed through a riffle splitter. One-hundred grams of material from the splitter were milled in a ball mill for approximately 8 minutes to create particles that would pass through a 100-mesh screen. Two grams of material less than 100-mesh were placed in a McCrone micronizing mill with $10 \mathrm{~mL}$ of 2-propanol for 4 minutes, which reduced the particle size to near 1 micron. The slurry was dried overnight. A 2-gram aliquot of the dried sample was passed through a 60-mesh sieve and then side packed into a sample holder for analysis. Samples were analyzed with a PANalytical Xpert Pro-MPD X-ray Diffractometer. Mineral phases were identified with Material Data Inc. Jade 9.1 software (http:// www.materialsdata.com/) by using "International Centre for Diffraction Data's 2009-PDF-4" and "National Institute of Standards and Technology FIZ/NIST Inorganic Crystal Structure Databases" (William Benzel, U.S. Geological Survey, written commun., 2010).

\section{Quality Assurance}

Because of the low groundwater production, the traditional three well-bore volumes of water could not be purged prior to sampling. Instead, samples were obtained by using low-flow pumps designed to minimize mixing of stagnant casing water within the targeted screened interval of water, and sampling began when field parameters met stability criteria as defined in the USGS field manual (U.S. Geological Survey, variously dated). All samples met the stability criteria, with the exception of the SC criteria of plus or minus $( \pm) 3$ percent not being met for wells 728 and 731 (SC variability of 6.1 percent and 24.1 percent, respectively) and the DO criteria of \pm 0.2 milligrams per liter $(\mathrm{mg} / \mathrm{L})$ not being met for well 817 (DO variability of $0.4 \mathrm{mg} / \mathrm{L}$ ). To assess the quality of the laboratory data for major ions, the anion and cation data were evaluated for ion electrical balance. Anion and cation data were not obtained for wells 728,824 , and 817 because of limitations of available water. In 3 of 8 samples, the anions and cations did not balance within a 5-percent difference; all but 1 sample (from well 603) were within a 10-percent difference. Well 603 had a negative excess, which could result because ammonium was not measured in this study, but historically this well has had high ammonium concentrations (U.S. Department of Energy, 2000).

Method blanks (MBs), laboratory-control samples and duplicates (LCSs and LCSDs), and matrix-spike and duplicate (MS and MSD) samples are laboratory quality-control samples that were analyzed with the environmental samples to evaluate the quality of the metals, inorganic anions, dissolved solids, and alkalinity results. Two MB samples, containing analytefree water, were analyzed and reported as having no detections above the reporting limit, indicating that no contamination was introduced during the preparation and analysis of the samples. Two LCSs and LCSDs were prepared with certified amounts of the target analytes and analyzed with the environmental samples. All of the recoveries are reported to be within 10 percent of the expected concentration, and relative percent differences (RPDs) between the LCS and LCSD were within 3 percent, indicating that the batch preparations and analysis are capable of making accurate measurements. The MS and MSD samples were prepared by adding a certified amount of the target analyte to a fraction of the environmental sample to determine if the matrix affected the accuracy of the measurements. The MS and MSD recoveries were all within the quality criteria for each analyte, and the RPDs between the MSD samples were under 3 percent.

Replicate samples were collected for CFC analysis at all wells. The RPD was used to determine the variability in the sample, and values greater than 20 percent were flagged as estimated and interpreted with caution. Two samples, from wells 813 and 815 , had two or more CFCs with replicate RPDs greater than 20 percent. Both samples had anomalous CFC-12 analytical results (well 813 had a value above the measured atmospheric equilibrium concentrations indicating contamination, and well 815 had peak interference). The 
concentrations of CFC-12 and CFC-113 in well 841 also indicated contamination from unidentified sources.

Replicate samples were collected for dissolved-gas analysis at two wells; one analysis in each replicate had an RPD greater than 20 percent (methane in well 728 and $\mathrm{O}_{2}$ in well 815). Because the nitrogen and argon values were excessively low, dissolved-gas concentrations could not constrain recharge temperatures for CFC model dates; therefore, simply the presence or absence of dissolved gases was used as a constraint for conceptualizing hydrologic processes.

\section{Statistical Methods}

Correlations between two variables were investigated for significance using the nonparametric Kendall rank correlation coefficient (Helsel and Hirsch, 2002). For this report, a correlation was considered statistically significant if the calculated $p$-value was less than 0.05 . In selected cases when the relation between two variables appeared linear and an estimate of the equation describing the relation was desired, the coefficient of determination $\left(\mathrm{R}^{2}\right)$ was used to determine the linear correlation from a least squares regression analysis. $\mathrm{A} \mathrm{R}^{2}$ linear correlation greater than 0.8 was considered strong, whereas a $\mathrm{R}^{2}$ correlation of less than 0.5 was considered weak.

\section{Source of Groundwater Beneath Many Devils Wash}

The alluvial structure of Many Devils Wash resembles that of many arroyos in the greater San Juan Basin, with several geomorphic units that include bedrock outcrops on mesas, strath terraces created in the Pleistocene, and channel fill occurring in the Holocene (Smith, 1992). Many Devils Wash drains an area of about $11.5 \mathrm{mi}^{2}$ and flows about 6 mi from south to north. The final 2,500 ft of its course to the confluence with the San Juan River is a narrow channel incised through a loess-filled valley and into Mancos Shale bedrock. This deep-channel incision begins at what is referred to as the "knickpoint," and is advancing southward by erosional headcutting. South (upstream) of the deepchannel incision, the channel of Many Devils Wash is much wider than downstream from the knickpoint and is cut into the loess deposits. The East Fork enters Many Devils Wash approximately $600 \mathrm{ft}$ upstream from the knickpoint (fig. 4). The East Fork drains an area of $1.6 \mathrm{mi}^{2}$ and is dry except during infrequent storm events. Tributary 1 parallels Many Devils Wash south-southwest from its junction with the East Fork (fig. 4) and is also incised into loess deposits.

Three potential sources of the groundwater that results in the seeps in the Many Devils Wash are: (1) groundwater flowing from the terrace deposits that underlie the disposal cell, (2) groundwater upwelling from the deep aquifers underlying the Mancos Shale units, and (3) focused recharge of precipitation in the channels and topographic depressions in the Many Devils Wash watershed. This section of the report evaluates these potential sources by describing the documented hydrogeologic framework, detailing observational and qualitative evidence, and reporting and interpreting the results of the groundwater age-dating tracers. In order to address the purpose of this study, it is first necessary to understand and compare features of background or natural groundwater quality relative to groundwater that may have been affected by the activities at the Shiprock Disposal Site.

\section{Groundwater-Well Classification}

Because pre-Mill (1954) groundwater-quality data do not exist for the site, post-Mill groundwater-quality data cannot be compared to naturally occurring conditions. Instead, groundwater-quality samples were collected from wells and (or) springs in the surrounding areas that were not affected by the Mill, and the resulting data are compared with data for wells in and near the Shiprock Disposal Site. The natural, or background, water-quality data were collected by the DOE from groundwater seeps and springs in the Upper Eagle Nest Arroyo and Salt Creek Wash (fig. 1) (U.S. Department of Energy, 2012a), and these sites are referred to as "analog sites" in this report. The analog sites are located within $10 \mathrm{mi}$ of Many Devils Wash, but north of the San Juan River, making them hydraulically removed from the Shiprock Disposal Site and related activities. The two arroyos drain the same silt loam soils as found in the Many Devils Wash watershed (Natural Resources Conservation Service, 2015), and they similarly downcut into the underlying weathered Mancos Shale (New Mexico Bureau of Geology and Mineral Resources, 2003).

In order to compare the groundwater from various wells to groundwater that has been affected by Mill activities, a classification system was developed to determine which site wells were most likely affected. Groundwater wells were initially classified as Mill-affected by the proximity of the well to the former Mill and uranium (U) concentrations. However, because of high $U$ concentrations measured in most of the wells, and the large range in $U$ concentrations on the terrace near the former Mill, the ${ }^{234} \mathrm{U} /{ }^{238} \mathrm{U}$ (uranium isotope) alphaactivity ratios (ARs) also was used to further classify wells as Mill affected.

The choice to use ARs to distinguish between Millaffected wells and background wells is based on work by Zielinski and others (1997), who demonstrated that the ${ }^{234} \mathrm{U} /{ }^{238} \mathrm{U}$ ARs could distinguish between the $\mathrm{U}$ derived from weathering of local aquifer minerals and the $U$ derived from processing mills. Zielinski and others (1997) observed that most natural groundwater has a ${ }^{234} \mathrm{U} /{ }^{238} \mathrm{U}$ AR greater than 1.0 , with typical values in the range of $1-3$, but that values in excess of 10 can occur. In contrast, the raffinate contains residual amounts of $U$ originally brought into solution by the reaction of the $U$ ore with strong oxidizing solutions of acid 
or alkali. This $\mathrm{U}$ is derived from a mixture of materials with an AR near 1.0 because it presumably has not been exposed to highly oxidizing conditions in the natural aquifer environment. With these considerations, $U$ ores that are processed in a mill should have an estimated time-integrated average AR of $1.0 \pm 0.2$, and the raffinate should retain the $\mathrm{U}$-isotope composition of the processed ore because neither the rapid, nearly complete dissolution of $U$ from crushed ore nor further chemical processing of the leachate will promote any isotopic fractionation (Zielinski and others, 1997).

Uranium naturally exists as three isotopes: on a mass basis, (1) 99 percent of $U$ exists as ${ }^{238} U$, (2) 0.7 percent of $\mathrm{U}$ exists as ${ }^{235} \mathrm{U}$, and (3) only 0.0054 percent of $\mathrm{U}$ exists as ${ }^{234} \mathrm{U}$; however, because ${ }^{234} \mathrm{U}$ has a specific activity that is $18,600,000$ times greater than its parent, ${ }^{238} \mathrm{U}$, it generally contributes an equal or slightly greater amount of radioactivity in groundwater (Sherman and others, 2007). This condition of equal activity is referred to as "secular equilibrium." Since the formation of the Earth, the total mass of each of the $U$ isotopes has been decreasing, but because of the relatively short half-life of the ${ }^{234} \mathrm{U}$ isotope compared with the age of the Earth, all of the primordial ${ }^{234} \mathrm{U}$ has been depleted. The ${ }^{234} \mathrm{U}$ is a daughter product of the ${ }^{238} \mathrm{U}$ decay series; thus, in a closed system, isolated from oxic weathering for approximately 1 million years, the alpha activities of the parent ${ }^{238} \mathrm{U}$ and daughter ${ }^{234} \mathrm{U}$ isotopes will become equal (Sherman and others, 2007). The preferential dissolution of ${ }^{234} \mathrm{U}$ in an open system is responsible for the slightly enriched activity ratios (near 1.4) in the oceans and many surface waters (Kronfeld and Adams, 1974). In order to achieve the high activity ratios (greater than $[>]$ 1.5) found in many groundwaters, Kigoshi (1971) offered an additional mechanism to the preferential dissolution that he referred to as "alpha recoil." Unlike lighter elements, the fractionation of the $U$ isotopes is not a function of the mass differential; instead, isotopic variations occur because of physical processes related to the nuclear decay of each isotope (Kronfeld and Adams, 1974). Alpha recoil refers to the fractionation of ${ }^{238} \mathrm{U}$ and ${ }^{234} \mathrm{U}$ during radioactive decay, which results from the displacement of a ${ }^{234} \mathrm{U}$ atom from the site of its parent ${ }^{238} \mathrm{U}$ atom. When ${ }^{238} \mathrm{U}$ decays to thorium-234 $\left({ }^{234} \mathrm{Th}\right)$ by alpha decay, the nucleus can be recoiled out of the mineral and into the groundwater. The ${ }^{234} \mathrm{Th}$ decays through protactinium-234 to ${ }^{234} \mathrm{U}$, resulting in an excess of ${ }^{234} \mathrm{U}$ in the groundwater compared to ores containing high concentrations of U (Zielinski and others, 1997). Ores that have not been subjected to major oxidative leaching within the last million years approximate closed systems and are in radioactive (secular) equilibrium (Zielinski and others, 1997). Longer groundwater residence times have been shown to increase the U concentrations in groundwater (Szabo and Zapecza, 1991; Tricca and others, 2000). Tricca and others (2000) argued that long groundwater-flow distances or low water velocities can increase not only the $\mathrm{U}$ concentrations in the groundwater but also the ${ }^{234} \mathrm{U} /{ }^{238} \mathrm{U}$ AR.
The activity of the isotopes of $U\left({ }^{234} U{ }^{238} U\right)$ in groundwater reported by DOE (U.S. Department of Energy, 2000; 2012a) for selected terrace and Many Devils Wash groundwater wells, as well as for the nearby analog sites, are plotted with the $U$ concentrations on figure 6 .

The wells classified as Mill affected because of their location (fig. 4) and (or) $U$ concentrations (table 1) have an AR of 1.2 or less. However, because most of the groundwater samples have $\mathrm{U}$ concentrations above the EPA Maximum Contaminant Levels (MCLs) (U.S. Environmental Protection Agency, 2009), it is difficult to characterize an affected well solely on the basis of $U$ concentrations. For example, two wells (730 and 830) that exhibited $U$ concentrations less than $0.01 \mathrm{mg} / \mathrm{L}$ are located next to the disposal cell and are considered affected by the Mill on the basis of the AR. One exception to the 1.2 AR criterion is well 602. Because of its proximity to the disposal cell and high $\mathrm{U}$ concentrations relative the analog sites, the well was included in the Millaffected group despite having an AR of 1.39. It is important to note that this classification system does not imply absolutes; in other words, that there can be effects outside the wells identified as affected. The strong scientific basis for the classification system, however, was deemed as an appropriate way to classify the wells to allow for comparing and contrasting the attributes of wells that have been affected by the Mill.

\section{Hydrogeologic Framework}

The hydrogeologic framework, as it relates to the potential sources of groundwater beneath Many Devils Wash, is presented by first describing each of the possible sources of groundwater beneath Many Devils Wash. The isotopic compositions of the hydrogen and oxygen in the groundwater in the area are also presented and compared with the composition of possible end members. Finally, the hydraulic gradients are examined to assess the potential for each of those sources.

\section{Potential Sources of Recharge to the Area of Many Devils Wash}

The potential sources of recharge to the area of Many Devils Wash are introduced in this section. Evidence is discussed that supports or does not support the identified source.

\section{Terrace Groundwater System}

There is no documented evidence to support or disprove the existence of a shallow groundwater table in the alluvial deposits on the terrace beneath the disposal cell or in Many Devils Wash prior to startup of the Mill. Since the Mill began operations in 1954, several sources of potential groundwater 


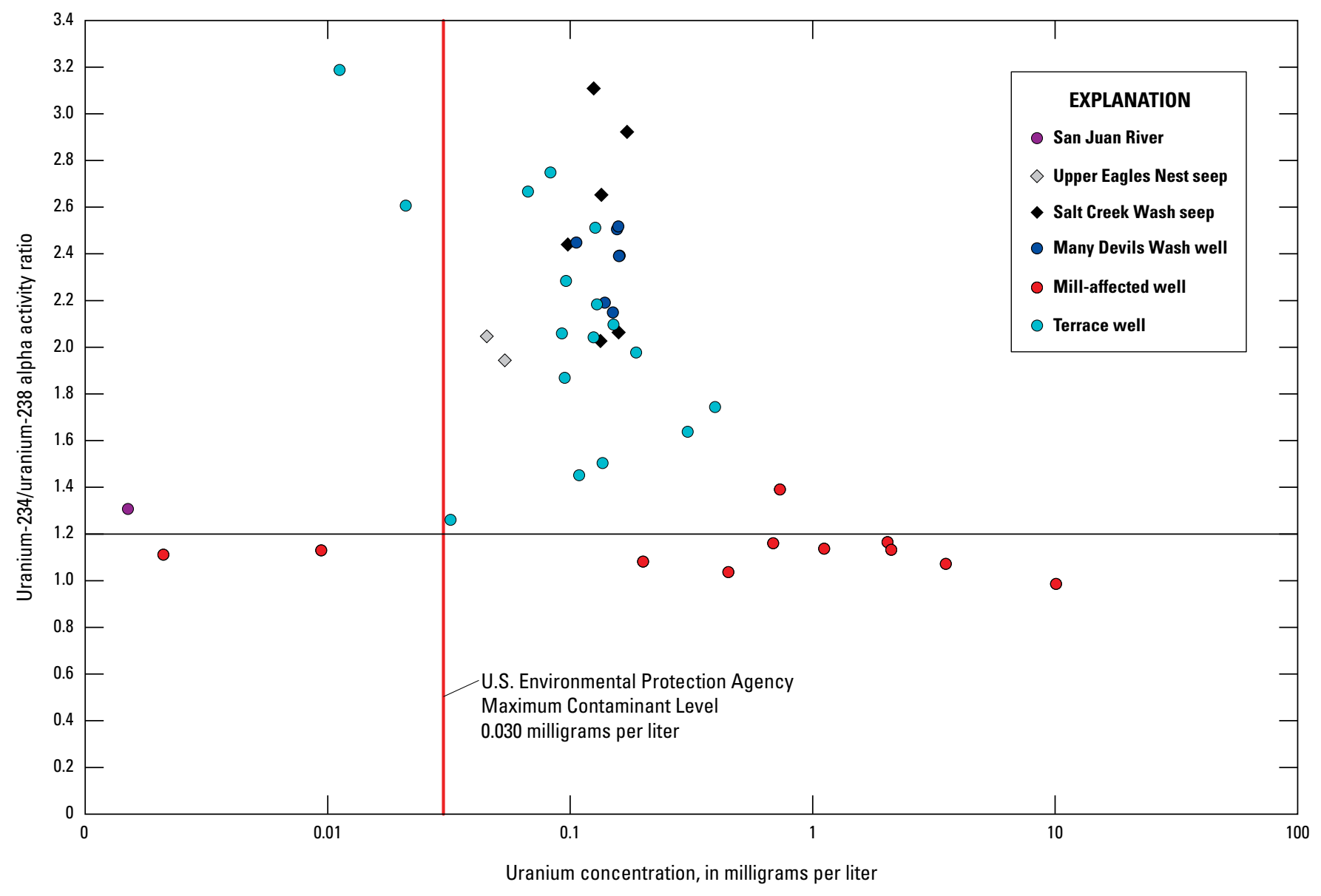

Figure 6. Uranium-234/238 alpha activity ratios and concentrations of uranium in groundwater at and near the Shiprock Disposal Site, Shiprock, New Mexico.

recharge have been introduced to the terrace. The artesian well 648 (fig. 4) was completed in the Morrison Formation in 1961 and has discharged groundwater into Bob Lee Wash continuously since it was drilled. This water may infiltrate into the arroyo bed and recharge the terrace deposits. Irrigation water for agriculture was diverted from the San Juan River and conveyed to the terrace west of the Shiprock Disposal Site between the 1960s and the late 1990s. Other potential anthropogenic sources of groundwater recharge include leaking water and sewer lines and domestic septic systems of nearby residences. Seepage from irrigation to suppress dust during surface remediation and activity (including excavating and washing gravels) associated with the Navajo Engineering and Construction Authority (NECA) gravel pit east of the disposal cell between about 1970 and 2009 may have introduced water into the shallow aquifer following the closure of the Mill.

Regardless of whether groundwater existed in the terrace before Mill operations, the process water is considered to be a major source of groundwater recharge into the terrace groundwater system. The process water created a groundwater mound under the Mill that migrated radially outward (U.S. Department of Energy, 2000). Although the Mill is no longer a source for groundwater recharge, the draining of encapsulated materials and leakage (estimated at $5 \mathrm{gal} / \mathrm{min}$ ) through the disposal cell is thought to be a continuing source of recharge (U.S. Department of Energy, 2012c). The DOE states that slimes (fine-grained tailings) within the disposal cell that directly overlie native terrace deposits have been nearly saturated to saturated during recent investigations (U.S. Department of Energy, 2012c).

All of the process wastes from the Mill were pumped to unlined ponds. The spent-ore slurry was pumped to the tailings piles located east of the Mill between the Mill and the river, and raffinate from the solvent extraction circuit and yellow cake filtrate was pumped to ponds on the south side of the Mill (fig. 2) (Public Health Service, 1962). According to the Public Health Service (1962) stream survey, a major portion of the seepage thought to originate from the waste ponds discharged in various gullies and washes near the site where 
alluvium has been eroded from the bluff. The stream survey reported that the highest concentrations of gross radiological activity were found in seepage nearest the tailings piles (760 to 860 picocuries per liter $[\mathrm{pCi} / \mathrm{L}]$ for alpha and 1,400 to 1,800 $\mathrm{pCi} / \mathrm{L}$ for beta), whereas seeps farthest from the piles exhibited substantially lower gross radiological activity ( 13 to $70 \mathrm{pCi} / \mathrm{L}$ for alpha and 100 to $860 \mathrm{pCi} / \mathrm{L}$ for beta). One important notation in the report is that Many Devils Wash was not sampled because it was reported as dry (Public Health Service, 1962). It is not clear from the report if the dry conditions refer simply to the lack of water to sample at the confluence or if there was an attempt to look upstream for seeps.

A surface geophysical survey in 1996 concluded that contaminant transport in the terrace appeared to be controlled by the top of bedrock surface features; the denser groundwater associated with high dissolved solids coincided with depressions in the top of bedrock surface (U.S. Department of Energy, 1996). A high-conductivity geophysical response resulted from high concentrations of $\mathrm{SO}_{4}$ and $\mathrm{NO}_{3}$ salts in the groundwater that appeared as a "halo" around the cell (U.S. Department of Energy, 1996). The 1996 geophysical study concluded that Mill contaminants leaked from the unlined ponds and produced a groundwater mound that moved outward from beneath the disposal cell (U.S. Department of Energy, 1996).

\section{Deep Groundwater Upwelling}

The USGS RASA investigation of the San Juan structural basin concluded that the deep aquifers in the Early Cretaceous and Jurassic sedimentary layers discharge to the San Juan River somewhere in the Four Corners region (Levings and others, 1996). Discharge from these lower aquifers could provide a possible source of water to the shallow aquifer through vertical leakage or preferential flow through fractures or joints. That study also concluded that the Mancos Shale is a confining layer for those units, and by extension, vertical leakage into overlying alluvium should be more restricted where the Mancos Shale is thicker. As reported in the "Introduction" section, there are $218 \mathrm{ft}$ of Mancos Shale between the alluvium and the Gallup Sandstone and another $685 \mathrm{ft}$ between the Gallup Sandstone and the Dakota Sandstone.

Artesian flow from well 648 demonstrates that an upward groundwater gradient between the Morrison Formation and land surface exists at the Shiprock site and suggests that upward groundwater flow from deep aquifers could be a potential source of groundwater beneath Many Devils Wash; however, other evidence indicates that upward flow through the Mancos Shale at the site does not occur. Several wells installed about 2 mi east of the site in the Chaco River watershed (fig. 1) are completed more than $30 \mathrm{ft}$ into the Mancos Shale and have always been dry (U.S. Department of Energy, 2014). In addition, the nested wells installed less than $200 \mathrm{ft}$ to the north and northeast of the disposal cell (fig. 4) (including wells 824 and 825) generally show a downward gradient or went dry soon after the well was developed. These wells are screened between 70 and $190 \mathrm{ft}$ into the Mancos Shale. Finally, a geophysical survey imaged the escarpment to look for preferential pathways from the terrace to the flood plain through fractures. The results indicate that few fractures are present and that there was no indication of preferential vertical flow paths (U.S. Department of Energy, 1996); therefore, although the potential exists for groundwater in the underlying aquifers to move upward into the shallow groundwater at the site, there is no hydrologic or geophysical evidence to suggest that this is occurring.

Deep groundwater contributions to the shallow groundwater in Many Devils Wash may result from abandoned oil test wells (Craig Goodknight, U.S. Department of Energy, written commun., 2015). A search of well records at the New Mexico Oil Conservation Division yielded one well (Navajo 5032-1) that was within the Many Devils Wash watershed (New Mexico Oil Conservation Division, 2015). The well, drilled in 1968, is about 1 mi upstream from well 1159 in Many Devils Wash. According to records, the hole was drilled to a depth of $1,397.5 \mathrm{ft}$ and "the hole got wet" at $558 \mathrm{ft}$. The well was plugged and abandoned within several days of reaching total depth.

\section{Recharge from Precipitation}

Another potential source of groundwater recharge to Many Devils Wash is the focused recharge of precipitation. Focused recharge is often the only mechanism for recharge from precipitation in arid and semiarid climates where evaporation rates exceed precipitation rates (Stone, 1986; Wood and Sanford, 1995). In this case, precipitation in the Many Devils Wash watershed would collect in the channels and topographic low points, infiltrate into the alluvium, and then flow north along the erosional bedrock contacts to and along the axis of the channel.

If the source of water to Many Devils Wash is precipitation, an estimate of recharge can be obtained by using a mass-balance approach with appropriate assumptions. By dividing the reported discharge of $0.3 \mathrm{gal} / \mathrm{min}$ from the seeps in Many Devils Wash (U.S. Department of Energy, 2000) by the reported drainage area of $11.5 \mathrm{mi}^{2}$ (U.S. Department of Energy, 2011a), an annual recharge rate of a little less than $0.0008 \mathrm{in} / \mathrm{yr}$ is estimated (about 0.01 percent of the average annual 7.0 in. of precipitation per year over the watershed). This estimate assumes minimal change in aquifer storage and that all of the water infiltrating through the unsaturated zone past the root zone discharges through the seeps at the downstream end of Many Devils Wash. If water was discharged elsewhere, such as to lower geologic units or to transpiration farther down the flow path, the annual recharge would be greater than this estimate. This value is considered reasonable given the large loss of water to evapotranspiration that is taking place in the watershed. 
A second estimate of recharge is calculated with a chloride (Cl) mass-balance approach (Anderholm, 1994; Wood and Sanford, 1995). Recharge is estimated by using the following relation:

$$
Q=P\left[C l_{p}\right] /\left[\mathrm{Cl}_{g w}\right]
$$

where

$$
\begin{aligned}
& Q \quad \text { is the recharge, in inches per year; } \\
& P \quad \text { is the average annual precipitation rate, in } \\
& \text { inches per year; } \\
& {\left[\mathrm{Cl}_{p}\right] \text { is the average concentration of } \mathrm{Cl} \text { in }} \\
& \text { precipitation, in milligrams per liter; and } \\
& {\left[\mathrm{Cl}_{\mathrm{gw}}\right] \text { is the } \mathrm{Cl} \text { concentration (table 1) in Many }} \\
& \text { Devils Wash groundwater, in milligrams } \\
& \text { per liter. }
\end{aligned}
$$

The average concentration of $\mathrm{Cl}$ in precipitation was calculated from records at the Cuba, Bandelier, Painted Desert, Mesa Verde, and Canyonlands National Atmospheric Deposition Program (NADP) sites over their respective periods of record (National Atmospheric Deposition Program, 2014). The average $\mathrm{Cl}$ wet deposition concentration was determined to be $0.11 \mathrm{mg} / \mathrm{L}$. Because of the controversy surrounding its measurement, the dry deposition contribution to the total $\mathrm{Cl}$ deposition is often estimated or not addressed at all (Sterling, 2000). Dry deposition is currently not reported for NADP sites and is no longer measured; however, this parameter was measured at all NADP stations until 1984 when it became optional and then was eliminated altogether in 1998. For this study, the dry component estimate of the $\mathrm{Cl}$ deposition was chosen on the basis of an analysis by Sterling (2000). Sterling (2000) calculated the spatial distribution of dry deposition from the measurements made from the dry collectors at NADP sites for work on ${ }^{36} \mathrm{Cl} / \mathrm{Cl}$ ratios across the United States. In that work, the dry fraction ranged between 30 and 50 percent of the total $\mathrm{Cl}$ deposition for northwestern New Mexico. The estimated dry deposition for this study was chosen to be 67 percent of the calculated wet deposition (equivalent to 40 percent of the total, or $0.18 \mathrm{mg} / \mathrm{L}$ ). The sum of the dry and wet deposition $(0.29 \mathrm{mg} / \mathrm{L})$ is similar to the bulk precipitation value of $0.21 \mathrm{mg} / \mathrm{L}$ reported for the Sevilleta National Wildlife Refuge in central New Mexico from records between 1989 and 1995 (Plummer and others, 2004). The Cl mass-balance approach yields a recharge rate of $0.0011 \mathrm{in} / \mathrm{yr}$ by using the average $\mathrm{Cl}$ concentration in all of the Many Devils Wash wells. The range of recharge estimates for the minimum and maximum $\mathrm{Cl}$ concentrations measured in the groundwater samples from Many Devils Wash is 0.0008$0.0011 \mathrm{in} / \mathrm{yr}$. The $\mathrm{Cl}$ mass-balance estimate is extremely robust and relatively insensitive to large differences in the average annual precipitation and average $\mathrm{Cl}$ deposition (Allison, 1988; Murphy and others, 1996). This estimate does assume that all $\mathrm{Cl}$ measured in the groundwater is derived from atmospheric sources; if there was a geologic component to the aqueous $\mathrm{Cl}$ concentrations, the calculated recharge estimate would be less than the actual recharge. Based on evidence presented in the following sections, it does not appear that there is a significant $\mathrm{Cl}$ contribution from the weathering Mancos Shale. Because Many Devils Wash is within a small watershed, it is reasonable to assume that there is no extra-basin transfer of $\mathrm{Cl}$ by way of surface flows. The similarity between the two massbalance estimations, only one of which has a precipitation term, suggests that precipitation is a plausible source of recharge to Many Devils Wash.

\section{Stable Isotopes of Water}

To further evaluate the potential sources of groundwater, ratios of the stable isotopes of water from various sources were compiled and compared. The isotopic composition (delta deuterium $[\delta \mathrm{D}]$ and delta oxygen- $18\left[\delta^{18} \mathrm{O}\right]$ ) of possible endmembers (sources) and the groundwater samples reported for the site were analyzed to spatially and temporally constrain likely sources of groundwater recharge. The variation in the isotopic composition of various potential recharge waters may help identify the source of recharge because of the mass-dependent fractionation of water isotopes (hydrogen and oxygen) resulting from temperature changes during the formation of precipitation and during evaporation prior to infiltration into the aquifer (Genereux and Hooper, 1998; Ingraham, 1998). This mass-dependent fractionation results because the various isotopic forms of water have different vapor pressures that results in precipitation associated with warmer temperatures containing a greater number of heavier isotopes (less negative $\delta^{18} \mathrm{O}$ and $\delta \mathrm{D}$ values) than precipitation associated with cooler temperatures (Ingraham, 1998). Similarly, the water that is left after evaporation will contain a larger number of heavier isotopes than water that has not been subjected to evaporation.

The stable-isotope ratios, reported in per mil (\%) relative to Vienna Standard Mean Ocean Water, of water from various sources are plotted in figure 7. Groundwater $\delta \mathrm{D}$ and $\delta^{18} \mathrm{O}$ values at the site plot below (to the right of) the Global Meteoric Water Line (GMWL) (Craig, 1961) (fig. 7). The overall position of the $\delta \mathrm{D}$ and $\delta^{18} \mathrm{O}$ values compared to the GMWL is typical of the arid and semiarid Southwestern United States climate where there is a large evaporation component to the water budget (Friedman and others, 1992). The samples were also compared with the Local Meteoric Water Line (LMWL) for the Upper Colorado River watershed (Kendall and Coplen, 2001) (fig. 7). The LMWL is an unweighted linear regression generated from 214 analyses of stream samples collected from 19 selected sites within the USGS National Stream Quality Accounting Network and Hydrologic Benchmark Network. The average $\delta^{18} \mathrm{O}$ and $\delta \mathrm{D}$ values of four samples collected from the San Juan River between the fall of 2010 and spring of 2012 (fig. 7) plot directly on the LMWL for the Upper Colorado River watershed. Like many rivers in the upper Colorado River watershed, the San Juan River is primarily sourced by high-elevation snowpack and precipitation and thus would be expected to have a lighter isotopic composition than the lower 


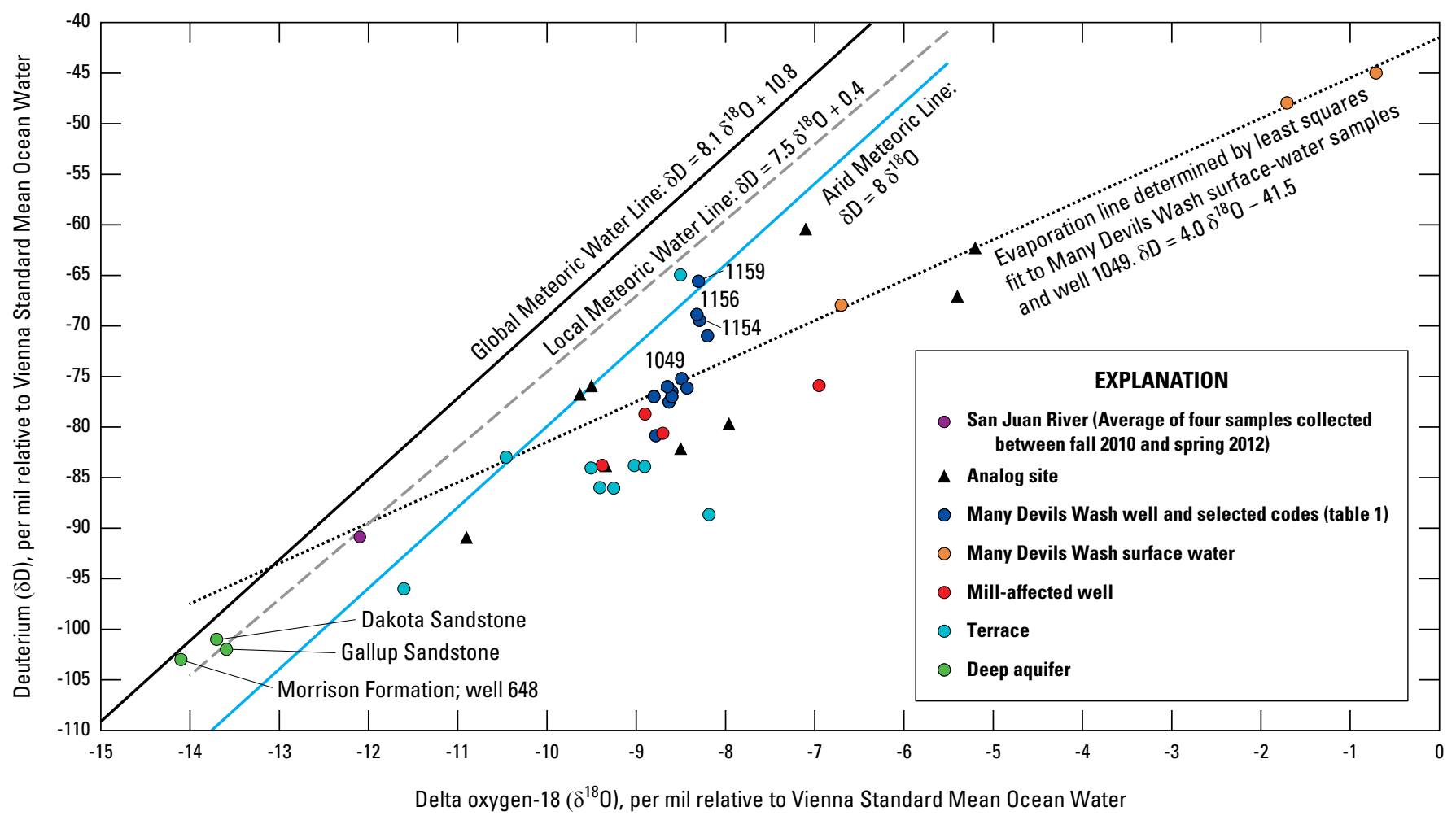

Figure 7. Delta deuterium $(\delta \mathrm{D})$ and delta oxygen-18 $\left(\delta^{18} 0\right)$ composition of selected samples from the study area at and near the Shiprock Disposal Site, Shiprock New Mexico.

elevation local precipitation. The $\delta^{18} \mathrm{O}$ and $\delta \mathrm{D}$ values (fig. 7) of groundwater from the shallow wells in the area plot below the LMWL, and a large number of values are even below the Arid Meteoric Line (AML) (Welch and Preissler, 1986). The heavier isotopic composition of these samples is a departure from the LMWL and the San Juan River and may indicate precipitation events at higher temperatures associated with the lower elevations of local recharge or the isotopic enrichment through evaporation.

The majority of the groundwater $\delta^{18} \mathrm{O}$ and $\delta \mathrm{D}$ values for the area fall below the AML, indicating the effects of evaporation on many of the samples. To assist in visually interpreting evaporation, the results of three surface-water samples collected from Many Devils Wash are presented, along with the groundwater samples (fig. 7). The evaporation line was determined by a least squares fit of the downstream Many Devils Wash well (1049) and the surface-water samples. The average isotopic composition in samples from well 1049 was chosen to represent the isotopic composition of groundwater that discharges from seeps prior to the influences of evaporation. This best-fit line provides visual representation of the likely evaporation signal. The evaporation signal (the $\delta \mathrm{D} / \delta^{18} \mathrm{O}$ slope; 4.0) calculated for Many Devils Wash falls within the expected range of evaporative effects (slopes between 3 and 6) according to Coplen and others (2000) and close to a slope of 5 reported by Phillips and others (2003) for samples from the main-stem Rio Grande and associate irrigation waters.

The range of $\delta^{18} \mathrm{O}$ and $\delta \mathrm{D}$ values, particularly along the AML, may result from seasonal influences on the stableisotopic composition of precipitation. Seasonal variations in the isotopic composition of local precipitation are caused by the differences in the temperature of condensation of atmospheric water vapor or from the source of the precipitation (Ingraham, 1998). Storms moving into the area primarily from the Pacific Ocean during the winter would have lighter compositions than thunderstorms originating primarily in the Gulf of Mexico or southern Pacific Ocean during the summer (Ingraham, 1998). Seasonal variation in stable isotopes in central and western New Mexico has been reported in $\delta^{18} \mathrm{O}$ values of precipitation and soil water (Breecker and others, 2009), $\delta \mathrm{D}$ in surface water (Plummer and others, 2004 ), and $\delta^{18} \mathrm{O}$ and $\delta \mathrm{D}$ values in groundwater (Robertson and others, 2013).

The $\delta^{18} \mathrm{O}$ and $\delta \mathrm{D}$ values of the groundwater samples collected from Many Devils Wash wells 1154, 1156, and 1159 in the upper watershed, which plot near the AML, suggest that groundwater recharge in Many Devils Wash is from local precipitation. Several other groundwater samples from wells on the terrace and the analog sites also have $\delta^{18} \mathrm{O}$ and 
$\delta \mathrm{D}$ values that lie near the AML, indicating that recharge from local precipitation events may be occurring elsewhere at the site. The evaporation line developed above suggests that many of the groundwater samples may reflect the seasonal variations in precipitation followed by evaporation; however, the evaporation line indicates that some groundwater samples may also have evolved (through evaporation when impounded) from San Juan River water. The San Juan River may be regarded as another potential end-member, particularly for the Mill-affected wells, because the San Juan River was the source of process water used at the Mill (Public Health Service, 1962).

Values for $\delta^{18} \mathrm{O}$ and $\delta \mathrm{D}$ were also compiled for groundwater samples from the deep aquifers underlying the Mancos Shale as part of the San Juan Basin RASA study conducted by the USGS (Dam, 1995). The average $\delta^{18} \mathrm{O}$ and $\delta \mathrm{D}$ values of 11 wells for the Gallup Sandstone and 2 wells for the Dakota Sandstone are displayed in figure 7, along with the $\delta^{18} \mathrm{O}$ and $\delta \mathrm{D}$ values for well 648 , which draws water from the Morrison Formation (well 18 in Dam [1995]). It should be noted that these samples are biased toward younger recharge because the majority of the wells are located at the margins of the basin where recharge is reported to occur. Based on the areal distribution of the stable isotopes in groundwater samples, Dam (1995) suggested that groundwater in the Morrison Formation that was farther than $10 \mathrm{mi}$ from the outcrops on the basin margins was recharged in the Pleistocene Epoch. Lower mean annual temperatures during the Pleistocene Epoch likely resulted in lighter isotopic ratios of precipitation entering the groundwater (Phillips and others, 1986). It is evident that the shallow groundwater at the site has a stable-isotope signature substantially more enriched in the heavier isotopes of oxygen and hydrogen than that of the deep aquifers, even when one considers the mixing of local recharge. Because there would be no opportunity for evaporation to occur if the source of water to the shallow aquifer were from the deep aquifers, the difference in the compositions strongly supports a recharge mechanism from the surface and not from deep upwelling.

Although the stable isotopes of water data do not confirm a single source of water for any of the well sets, they do indicate that the shallow groundwater around the site does not contain a large component of groundwater from the deep aquifers, which have a distinctively lighter isotopic composition. The heavier isotopic composition of the shallow groundwater samples relative to the San Juan River may indicate precipitation events associated with the lower elevations, especially when the composition falls close to a meteoric line; however, the isotopic enrichment of heavier isotopes in the groundwater because of evaporation prior to recharge makes it difficult to distinguish between local precipitation and evaporated San Juan River water in many of the wells. Making the distinction more difficult is the large variation in the isotopic composition of groundwater samples from the analog sites. The samples from the analog sites exhibit a variation that supports the large seasonal variation in the timing of recharge to each well as well as the effects of evaporation.

\section{Hydraulic Gradients}

Groundwater movement requires a potential gradient. Just as in other flow processes of mass or energy, groundwater moves from a higher potential to a lower potential at a rate that is proportional to the difference in the potential and the resistance to flow. The hydraulic gradient is defined as the change in hydraulic head over a distance.

\section{Terrace}

Groundwater elevations at the site are generally reported as undifferentiated between the alluvium and Mancos Shale because the majority of monitoring wells were constructed with the well screen set across both geologic units or with the borehole annulus not sealed across the contact, allowing for alluvial water to mix with Mancos Shale water in the well; therefore, a section diagram along a potential flow path, the east mill flow path, ( $A$ to $A^{\prime}$ 'in fig. 4 ) is presented instead of a potentiometric-surface map. The diagram in figure 8 shows the well construction in relation to the alluvium and Mancos Shale and the August 2012 groundwater elevations in the wells located between the disposal cell and Many Devils Wash. The construction information displayed in figure 8 includes the fill used in the well annulus and the location of the well screen and blank casing. Historical groundwater elevations in the monitoring wells between the disposal cell and Many Devils Wash are reported in table 3.

The groundwater hydraulic gradient between the disposal cell and Many Devils Wash indicated flow from the disposal cell in the three wells $(830,603$, and 731) measured between the disposal cell and Many Devils Wash before midyear 2001. The gradients were small between wells on the terrace (between 5.2E-04 and 2.4E-03) and slightly larger between the terrace and Many Devils Wash (well 1049) (about 5.0E02). Following a gap in data between 2001 and 2003, the gradient changed to a groundwater-elevation high centered around wells 603 and 731, giving groundwater the potential to flow west toward the disposal cell (between wells 603 and 731 to well 830), with an average gradient of $3.4 \mathrm{E}-03$, and to the east (between wells 603 and 731 to well 1059), with an average gradient of about 4.2E-04. The change in the direction of the potential gradient from wells 603 and 731 to 830 is the result of a decline in the groundwater elevation in well 830. The addition of two new monitoring wells (1058 and 1059 ) in 2000 provides more information about the gradient between wells 603 and 731 and Many Devils Wash. Well 1058 is located between well 731 and 1059 and has a groundwater elevation that fluctuates much more erratically than the other wells in the area but is consistently lower than the surrounding two wells. Well 1058 may be associated with an erosional feature because of its proximity to a buried channel. 


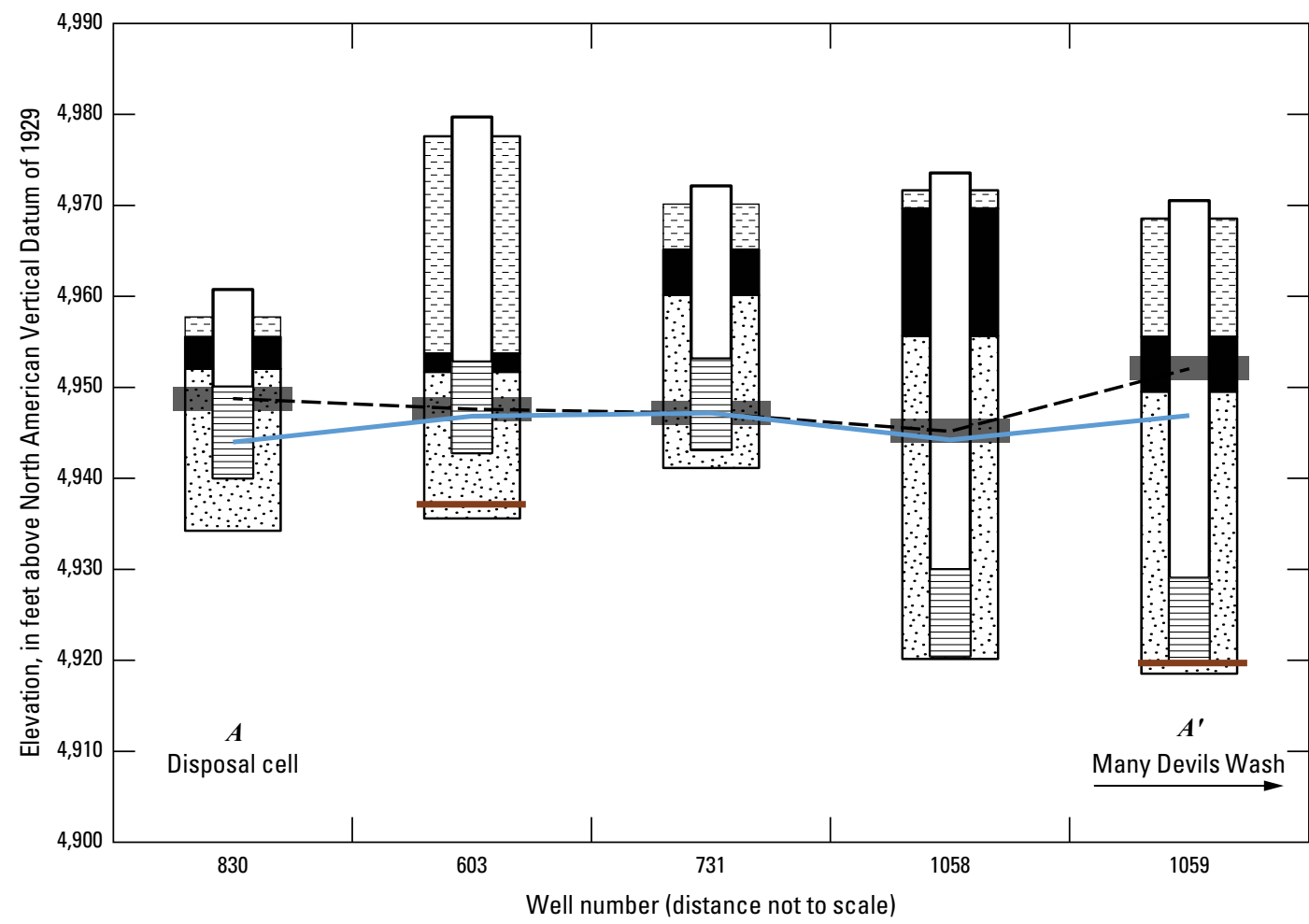

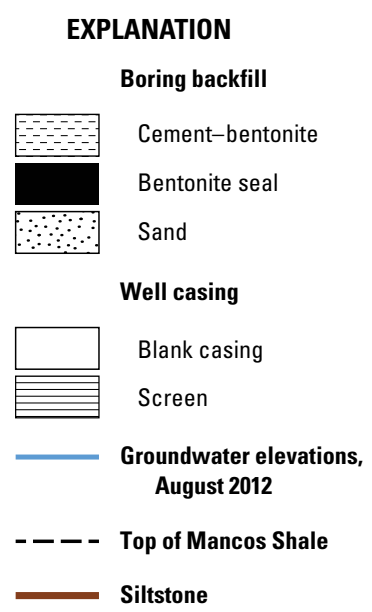

NOTE: Well locations and flow path $A$ to $A^{\prime}$ are shown on figure 4 .

Figure 8. Well construction relative to lithology and the August 2012 groundwater elevations in the monitoring wells along the east mill flow path between the disposal cell and Many Devils Wash at the Shiprock Disposal Site, New Mexico.

Table 3. Historical groundwater elevations in monitoring wells 830, 603, 731, 1058, and 1059 at the Shiprock Disposal Site, New Mexico. [Well locations shown on figure 5. NAVD 88, North American Vertical Datum of 1988; -, no data]

\begin{tabular}{cccccc}
\hline & \multicolumn{5}{c}{$\begin{array}{c}\text { Groundwater elevation } \\
\text { (feet above NAVD 88) }\end{array}$} \\
\cline { 2 - 6 } Date & $\mathbf{8 3 0}$ & $\mathbf{6 0 3}$ & $\mathbf{7 3 1}$ & $\mathbf{1 0 5 8}$ & $\mathbf{1 0 5 9}$ \\
\hline Dec. 1998 & $4,955.19$ & $4,951.52$ & $4,951.81$ & - & - \\
Mar. 1999 & $4,953.79$ & $4,951.66$ & $4,951.36$ & - & - \\
June 1999 & $4,954.34$ & $4,951.59$ & $4,951.34$ & - & - \\
Feb. 2000 & $4,953.30$ & $4,951.62$ & $4,951.36$ & - & - \\
June 2000 & $4,953.29$ & $4,951.56$ & - & $4,951.18$ & $4,950.66$ \\
Feb. 2001 & $4,952.51$ & $4,951.27$ & - & $4,947.55$ & $4,950.33$ \\
Aug. 2003 & $4,946.78$ & $4,951.37$ & $4,951.21$ & - & $4,950.53$ \\
Nov. 2003 & $4,947.15$ & $4,951.40$ & $4,951.60$ & - & $4,950.65$ \\
Mar. 2004 & $4,946.25$ & $4,951.30$ & $4,951.23$ & - & $4,950.40$ \\
Mar. 2005 & $4,947.22$ & $4,951.21$ & - & - & $4,950.60$ \\
Jan. 2006 & $4,946.68$ & $4,951.24$ & $4,950.98$ & - & $4,950.36$ \\
Mar. 2006 & $4,946.37$ & $4,951.10$ & $4,950.92$ & - & $4,950.32$ \\
June 2006 & $4,946.10$ & $4,950.99$ & $4,950.84$ & - & $4,950.14$ \\
Sept. 2006 & $4,946.25$ & $4,950.76$ & $4,950.49$ & - & $4,950.20$
\end{tabular}

\begin{tabular}{cccccc}
\hline & \multicolumn{5}{c}{$\begin{array}{c}\text { Groundwater elevation } \\
\text { (feet above NAVD 88) }\end{array}$} \\
\cline { 2 - 6 } Date & $\mathbf{8 3 0}$ & $\mathbf{6 0 3}$ & $\mathbf{7 3 1}$ & $\mathbf{1 0 5 8}$ & $\mathbf{1 0 5 9}$ \\
\hline Mar. 2007 & $4,946.14$ & $4,950.74$ & $4,950.69$ & - & $4,950.25$ \\
Sept. 2007 & $4,946.16$ & $4,950.56$ & $4,950.26$ & $4,928.40$ & $4,950.13$ \\
Mar. 2008 & $4,945.84$ & $4,950.26$ & $4,950.65$ & $4,941.20$ & $4,950.23$ \\
Sept. 2008 & $4,947.84$ & $4,951.21$ & $4,949.97$ & $4,949.19$ & $4,949.67$ \\
Mar. 2009 & $4,947.19$ & $4,950.75$ & $4,949.86$ & $4,930.92$ & $4,949.68$ \\
Sept. 2009 & $4,947.01$ & $4,950.29$ & $4,949.77$ & $4,942.84$ & $4,949.80$ \\
Mar. 2010 & $4,946.91$ & $4,949.90$ & $4,949.99$ & $4,946.33$ & $4,949.74$ \\
Aug. 2010 & $4,947.17$ & $4,949.88$ & $4,950.41$ & $4,946.61$ & $4,949.80$ \\
Mar. 2011 & $4,947.53$ & $4,950.31$ & $4,950.63$ & $4,947.36$ & $4,950.35$ \\
Sept. 2011 & $4,947.32$ & $4,950.01$ & $4,950.15$ & $4,947.79$ & $4,950.06$ \\
Mar. 2012 & $4,946.63$ & $4,951.55$ & $4,950.17$ & $4,948.18$ & $4,949.96$ \\
Aug. 2012 & $4,946.90$ & $4,949.75$ & $4,950.10$ & $4,947.16$ & $4,949.84$ \\
Mar. 2013 & $4,946.36$ & $4,949.76$ & $4,950.09$ & $4,948.10$ & $4,949.62$ \\
\hline
\end{tabular}


It is impossible to determine vertical hydraulic gradients between the alluvium and Mancos Shale because of the well construction (the wells are screened across lithologies). It has been suggested that the siltstone layer discussed in the "Lithology and Mineralogy" section may act as a vertical barrier to flow (U.S. Department of Energy, 2011a); however, it is not possible to determine if it may impart artesian conditions on groundwater in the Mancos Shale because well 1059 is screened across the layer and the sandpack in well 603 is across the contact.

\section{Groundwater Elevations Beneath Many Devils Wash}

In 2010 and 2011, the DOE drilled numerous wells and boreholes along the length of Many Devils Wash, Tributary 1, and East Fork (U.S. Department of Energy, 2011a, 2012b) (fig. 4). Groundwater elevations measured in these wells in June 2013 show that groundwater has the potential to flow from south to north along the axis of the Many Devils Wash channel (fig. 9).

Groundwater elevations in these wells coincide with the alluvium-Mancos Shale contact. The loess often has thin gravel layers that are typically at or near the base of the loess. The DOE described the occurrence of groundwater in borings that have a recognizable basal gravel layer as capable of producing more groundwater than borings with only loess; however, DOE cautioned that this relation is uncertain given that identification of the gravel layers was difficult during hand coring (U.S. Department of Energy, 2011a).

The hydraulic gradients presented in figures 8 and 9 demonstrate that groundwater presently has the potential to flow down the axis of the Many Devils Wash and, conversely, that groundwater located beneath the disposal cell is no longer able to flow toward Many Devils Wash. The current hydraulic potential likely does not represent conditions in the past. The groundwater regime during milling activities would most likely have been much different because of the influence of the groundwater mound (U.S. Department of Energy, 2000) underneath the waste ponds. (The waste ponds are underneath the disposal cell [fig. 4].)

\section{Observations of Hydrologic Conditions}

This section describes key observations that provide evidence of the likely hydrologic conditions and processes at the study site. The observations discussed in this section include the location of seeps in Many Devils Wash, the geomorphic structures that indicate water movement, and the type and distribution of vegetation.

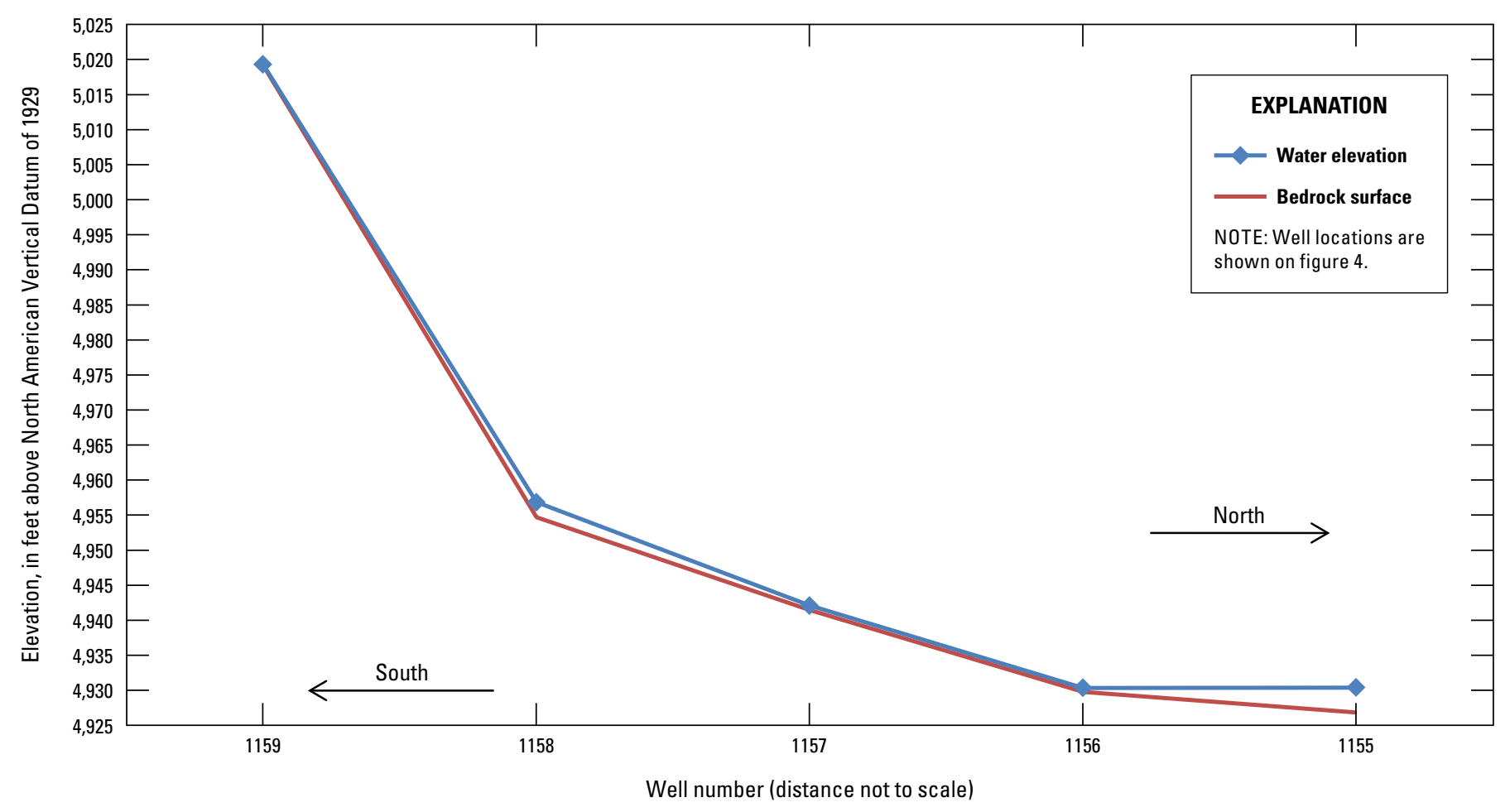

Figure 9. Elevation of groundwater and bedrock surfaces along Many Devils Wash, New Mexico, June 2013. 


\section{The Source of Groundwater and Solutes to Many Devils Wash at a Former Uranium Mill Site in Shiprock, New Mexico}

\section{Seep Observations}

Historically, groundwater elevations may have resulted in the potential for groundwater on the terrace, east of the disposal cell, to flow toward Many Devils Wash (table 3); however, seeps of groundwater issuing from the Mancos Shale have almost exclusively been observed on the east bank of Many Devils Wash (the bank opposite of the disposal cell). Seepage occurs along a line of seeps starting at the knickpoint (fig. 5) and extending northward for about $400 \mathrm{ft}$. Seepage also occurs upstream from the knickpoint at the confluence of East Fork with Many Devils Wash as indicated by the presence of efflorescent salts. Given this observational evidence, if the source of groundwater beneath Many Devils Wash is the Mill site, groundwater would have had to move down from the unlined tailings piles and raffinate ponds into the underlying Mancos Shale, then move laterally along bedding planes that dip gently eastward. To discharge only on the east side of the wash, the groundwater would move up under a pressure gradient created by the confining siltstone through a vertical joint system. The dip of the bedrock, along with the reported confining properties of the siltstone, could generate the quasiconfined conditions required to develop an upward pressure gradient on the east side of Many Devils Wash. However, this is in contradiction to early observations that groundwater movement was controlled by bedrock surface features (Public Health Service, 1962; U.S. Department of Energy, 1996).

Groundwater flowing across the top of the siltstone bed and discharging to Many Devils Wash is improbable because Many Devils Wash has downcut through the siltstone bed, and seeps have been observed almost exclusively on the east side of the channel. In addition, a buried channel located between the disposal cell and Many Devils Wash and roughly extending northwest from well 1058 to the escarpment (U.S. Department of Energy, 2000) may have intercepted groundwater moving along or through the bedrock features and redirected the flow north toward the San Juan River. It is interpreted that this was occurring in 1960 when the Public Health Service (1962) stream survey reported that the now buried channel was discharging water at $1.5 \mathrm{gal} / \mathrm{min}$ but there has been no evidence of recent flow.

\section{Piping}

Observational evidence for direct or focused recharge of precipitation can be seen in piping erosion referred to as "pseudokarst-type landscapes" by Parker (1963) (fig. 5). Parker (1963) describes piping as an erosional process occurring in drylands that is often associated with a lack of vegetation and gullying (indicating steep-gradient subsurface drainage). Piping may occur when cracks develop in desiccated soils and are subsequently inundated by water from thunderstorms that carry away the weakly or unconsolidated sediments (Parker, 1963). Groundwater sapping, as described by Leopold and others (1964), is the process of undercutting steep-walled arroyos from shallow groundwater seeping into the channel. The two erosional processes contribute to the visible geomorphic erosional features, such as towers, channel incision, and slope failures, in the recent loess deposits (U.S. Department of Energy, 2011a). These geomorphic features provide evidence that precipitation on the land surface is infiltrating into the loess and alluvial deposits in Many Devils Wash.

\section{Vegetation}

Along with the geomorphic evidence, site vegetation provides additional observational evidence for focused recharge of precipitation. Along the axes of the stream channels, the vegetation is much more prominent than in surrounding uplands. Deep-rooting species observed in the channels include Atriplex canescens (four-wing saltbrush), Asteraceae, spp. (rabbitbrush), Gutierrezia saraothrae (snakeweed), and Artemisia spp. (sage), as well as perennial grasses such as Oryzopsis hymenoides (Indian ricegrass), Sporobolus cryptandrus (sand dropseed), and Hilaria jamesii (galleta); there are even a few phreatophytes such as Tamarisk, spp. (salt cedar) present (Allison and Ashcroft, 2014). The root depths of these species found in the channels were observed to be between 25 and $35 \mathrm{ft}$ below the ground surface in the exposures of arroyo walls. These root depths are comparable to the depths to bedrock. In contrast, the upland vegetation is extremely sparse and primarily includes Atriplex confertifolia (shadscale) and Sclerocactus mesae-verdae (Mesa Verde cactus) (Allison and Ashcroft, 2014). The density and type of vegetation in the channel, relative to the upland vegetation, suggest that runoff is collecting in the arroyo and recharging the groundwater on a somewhat permanent basis. The absence of dense populations of phreatophytes or wetland species suggests that there is not a joint or fracture allowing for significant amounts of deep upwelling to recharge the shallow aquifer in the wash and diffuse vertical leakage from deeper aquifers would likely be captured by the lower elevation of the San Juan River channel.

\section{Groundwater Age-Dating Tracers}

Groundwater age-dating tracers were analyzed in samples collected by the USGS from selected wells around the site to determine the apparent ages of groundwater. The operational history of the Mill overlaps the changing atmospheric concentrations of anthropogenic tracers, providing an opportunity to distinguish between recent and pre-Mill sources of water to the aquifer.

\section{Tritium}

Tritium data from 10 wells sampled in November 2012 are presented in figure 10. The historical decay curves for the measured concentrations are plotted along with the ${ }^{3} \mathrm{H}$ concentrations in precipitation in Albuquerque, N. Mex. (Jurgens and others, 2012). The results and calculated decay curves are displayed logarithmically to aid in the visual interpretation. 


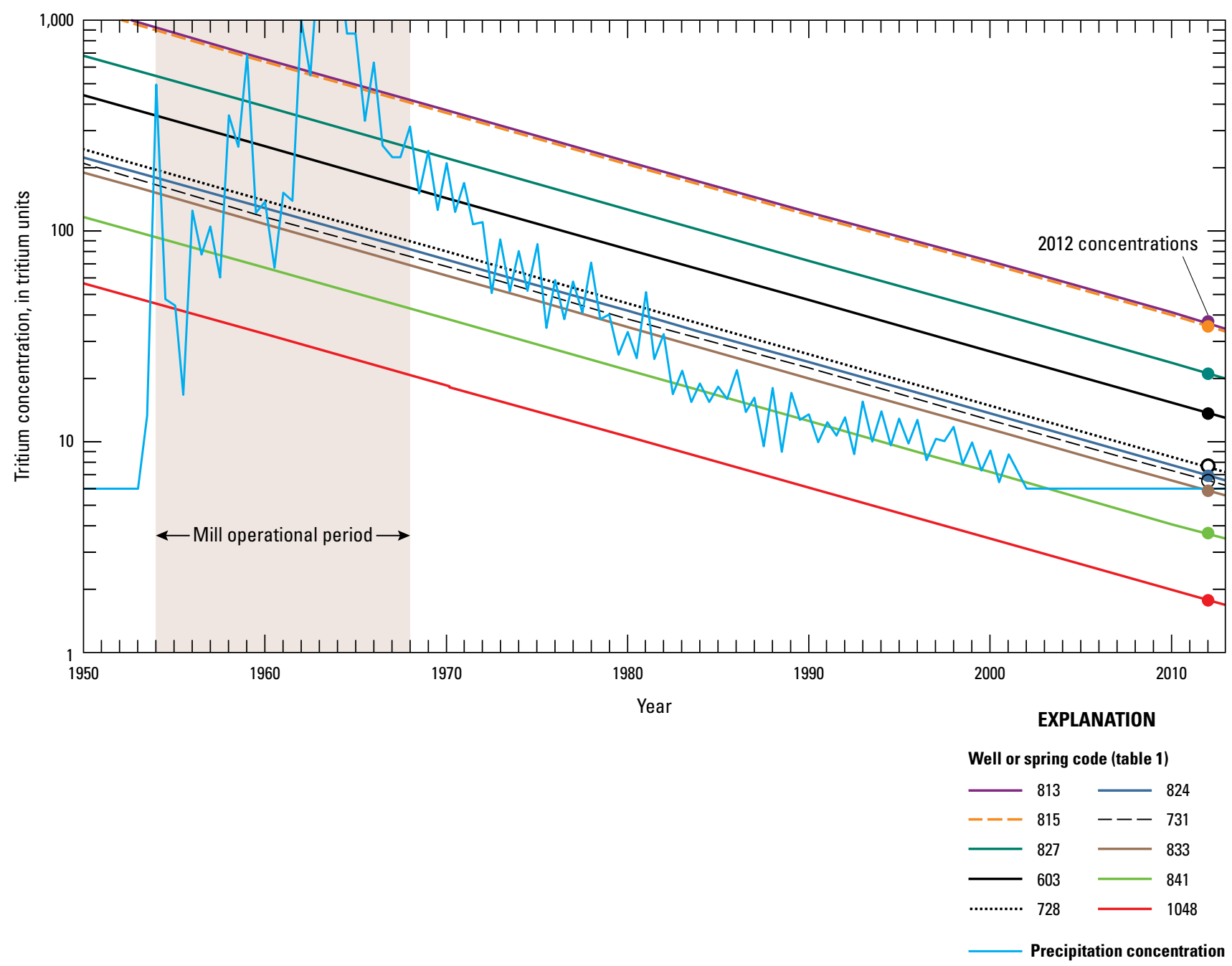

Figure 10. Tritium $\left({ }^{3} \mathrm{H}\right)$ values for groundwater collected from wells at the Shiprock Disposal Site, November 2012, with projected decay curves and precipitation concentrations measured in Albuquerque, New Mexico, 1950-2013.

Because the decay curves intercept multiple years of equivalent precipitation concentrations, the ${ }^{3} \mathrm{H}$ values cannot definitively determine the date that groundwater recharge occurred; however, the concentrations do constrain possible time periods when most of the water was recharged. The following interpretations assume piston-type flow. Given the site hydrogeology, primarily, the thin saturated thickness, piston-type flow is considered a reasonable estimate for the recharge conditions. The possibility of the mixing of different age waters is discussed throughout this section.

The ${ }^{3} \mathrm{H}$ concentrations measured in the 10 wells at the site ranged from 1.66 to $34.03 \mathrm{TU}$ (table 2). Because the greatest atmospheric ${ }^{3} \mathrm{H}$ values coincided with the time that the Mill was operating (fig. 10), groundwater with ${ }^{3} \mathrm{H}$ values greater than about 19 TU (in 2012-13) must have a large component that was recharged during the time the Mill was operating. The decay curve calculated from a ${ }^{3} \mathrm{H}$ concentration of $19 \mathrm{TU}$ in $2012-13$ is greater than the precipitation concentrations after about 1969 following the Mill closure and loss of remaining water in the raffinate ponds. It is also greater than concentrations before the Mill was operational and therefore groundwater could not have been recharged before or after the Mill began operations. Given the large amounts of water used in the $U$ processing, it is reasonable to assume that these concentrations indicate the presence of Mill-process water. The three greatest ${ }^{3} \mathrm{H}$ concentrations (34.03, 33.21, and $19.98 \mathrm{TU}$ ) were measured in samples collected from wells 813,815 , and 827 , indicating that the groundwater in these wells was recharged when the Mill was operating. All three wells are located west of the disposal cell (fig. 4), providing evidence that at least part of the process water from the Mill was moving west and by extension, flowing along the bedrock surface rather than strictly through bedrock bedding planes (that dip slightly to the east as noted in preceding sections). 
A review of the ${ }^{3} \mathrm{H}$ concentrations from groundwater samples reported by DOE (U.S. Department of Energy, 2012a) shows that concentrations above about $19 \mathrm{TU}$ occur exclusively in wells near the disposal cell; additionally, all three of the samples from the DOE dataset that had ${ }^{3} \mathrm{H}$ concentrations above $19 \mathrm{TU}$ (in 2011-12) had U activity ratios less than 1.2. The two values are not dependent, but considering the site history, they should be related. Given this relation, it is unclear why the samples from wells 813 and 815 would have such high ${ }^{3} \mathrm{H}$ concentrations but have ARs greater than 1.2 (the highest AR expected for Mill-processed U). One possible explanation is that groundwater in these wells represents a recharge source separate from the Mill. Another possible explanation is that the groundwater along this flow path may have passed through a reducing zone and lost some or all of the $\mathrm{U}$ from the Mill by precipitating out of solution. Another explanation may be that water along the flow path mixed with a more oxic recharge source or one that had more uraniumcomplexing carbonate, allowing for mixing with locally sourced $U$ with higher ARs. This explanation is supported by the fact that the bicarbonate concentrations in wells 813 and 815 are two of the greatest in the terrace wells (table 2), and ARs for the two wells (2.1 and 1.64) fall between the ARs for many of the Mill-affected wells and the analog sites (fig. 6 and table 1).

The relatively high concentration of ${ }^{3} \mathrm{H}(12.98 \mathrm{TU})$ in the sample from well 603, which is located southeast of the disposal cell, suggests that at least some of the groundwater was recharged during the Mill operation or in the 4 years following closure. The ${ }^{3} \mathrm{H}$ concentrations, along with the reported high concentrations of ammonium in well 603 (U.S. Department of Energy, 2000), indicate that some component of Mill-derived groundwater moved to the east of the cell. This evidence, however, is contradicted by the fact that the uranium AR in well 603 is one of the greatest (3.19) at the site (table 1). The ${ }^{3} \mathrm{H}$ concentration in well $731(6.12 \mathrm{TU})$, just to the southeast of well 603, is substantially lower than the sample from well 603 and is comparable to presentday precipitation concentrations. The large difference in concentrations between the two wells indicates that the Millage water has not flowed to well 731 or has been replaced by younger water. There is no reported AR value for well 731 to aid in the interpretation. One possible explanation for this difference is that well 731 is located near a buried channel that may have captured groundwater flowing from the former Mill across the bedrock surface. Another possibility is that the washing process, associated with the NECA gravel pit (see the "Potential Sources of Recharge" section), may have introduced a large quantity of water to the terrace groundwater east of the disposal cell following the Mill operations. Another possibility is that the ground surface to the east of the cell has been reworked and excavated to the alluvial gravels; the lack of overburden may currently allow for more recharge from precipitation.

Groundwater samples from three other wells $(824,833$, and 728) also had ${ }^{3} \mathrm{H}$ concentrations (5.54-7.19 TU) near the value for modern precipitation (around $6 \mathrm{TU}$ ), but intersections of the decay curves with the atmospheric concentrations indicate that recharge may also have occurred during and after the operational period of the Mill, until about the early 1980s. Multiple mixing scenarios might also be proposed where these dates can represent mixing between Mill-age water and younger (or older) water, making an interpretation of the groundwater age in these wells, on the basis of ${ }^{3} \mathrm{H}$ alone, difficult. Well 728 has been identified as having been affected by the Mill because of its uranium AR (1.08), suggesting that either younger water is mobilizing the Mill-sourced uranium or there is mixing of different-aged water. The groundwater sample from well 841 had a ${ }^{3} \mathrm{H}$ concentration $(3.43 \mathrm{TU}$ ) less than recent precipitation concentrations. The apparent age of the groundwater in well 841, based on where the decay curve intersects the atmospheric precipitation curve (fig. 10), places the groundwater age for this well near the start of Mill operations, possibly indicating the extent of the Mill-process water; however, the decay curve also intersects precipitation concentrations that are younger than the Mill-age water (possibly recharged between 1980 and 2000).

The ${ }^{3} \mathrm{H}$ concentration measured in the groundwater sample from well 1048 in Many Devils Wash was relatively low (1.66 TU), suggesting that it was recharged between 1953 and 1956, immediately before or at the very beginning of Mill operations. The ${ }^{3} \mathrm{H}$ concentration in well 1048 is similar to concentrations reported by DOE for other wells in Many Devils Wash. The concentrations reported by DOE (U.S. Department of Energy, 2012a) ranged from 1.09 to $1.61 \mathrm{TU}$ (table 1), with an average concentration of $1.3 \mathrm{TU}$. These concentrations could represent the initial pulse of Mill water (1954-56) or later Mill water (corresponding to higher ${ }^{3} \mathrm{H}$ values) mixed with a majority of pre-Mill water (with ${ }^{3} \mathrm{H}$ concentrations near zero TU). Because this small range of concentrations was measured in wells spaced over a mile apart in Many Devils Wash (1048, 1049, 1150, 1151, 1154,1156 , and 1159), it appears unlikely that all these wells would contain similar fractions of Mill-water recharge that occurred within a short period of time; therefore, these data suggest an alternate source of recharge to the groundwater beneath Many Devils Wash. If the source of recharge to Many Devils Wash was precipitation, as evidence presented in previous sections suggests, then recharge may occur anytime there is enough precipitation to cause a pulse of water to infiltrate; therefore, it is reasonable that groundwater in this area has a mixture of different-aged water that might include a large component of pre-1953 precipitation mixed with more recent water.

The tritium-age dates provide strong evidence that Mill-age water is present in several wells on the terrace (813, 815 , and 827 ) but also suggest that mixing of different-aged water may be occurring. The ${ }^{3} \mathrm{H}$ concentrations measured in the wells between the disposal cell and Many Devils Wash indicate that groundwater that recharged during Mill operations is not the dominant source of groundwater found beneath Many Devils Wash. 


\section{Chlorofluorocarbons}

The results of the CFC analysis on groundwater samples from eight wells are presented in figure 11, along with the North American atmospheric loading of CFC-12, CFC-11, and CFC-113 (Jurgens and others, 2012). As discussed in the "Study Methods" section, the recharge conditions of the groundwater sampled were assumed to be at an elevation of $4,980 \mathrm{ft}$ above NAVD 88 and at a recharge temperature of $55.3^{\circ} \mathrm{F}$.

Groundwater from all the wells sampled had detectable CFC concentrations, indicating that at least some fraction of water has recharged since the 1940s. There are some common characteristics in the measured concentrations among the wells. The CFC-11 concentration in every sample has a model date of recharge older than the other two CFCs. This pattern may be the result of reducing conditions, which preferentially degrade CFC-11 or the widespread contamination of CFC12 and $\mathrm{CFC}-113$ at the site. It was determined that there was contamination of CFC-12 in the groundwater samples from wells 815,813 , and 841 and of CFC-113 in well 841 based on the excessively high concentrations measured in these wells relative to atmospheric concentrations (these concentrations are not included in fig. 11).

Given the uncertainty in recharge temperature (which may yield an error in the model date of up to \pm 5 years), the CFC results generally support and help to further constrain the ${ }^{3} \mathrm{H}$ interpretations. Groundwater $\mathrm{CFC}$ concentrations in well 728 constrain the ${ }^{3} \mathrm{H}$ model dates by indicating that the groundwater likely recharged in the early to mid-1970s. Despite the contamination of CFC-12 in well 815, the other two CFC concentrations suggest similar, but slightly younger, recharge dates than well 728. The CFC concentrations in wells 813 and 817 suggest a recharge date in the 1970s, which does not agree particularly well with the reported ${ }^{3} \mathrm{H}$ model dates of the 1960s. Tritium and CFC concentrations indicate that groundwater sampled from well 833 was likely to have recharged in the early 1980s. The CFC concentrations in well

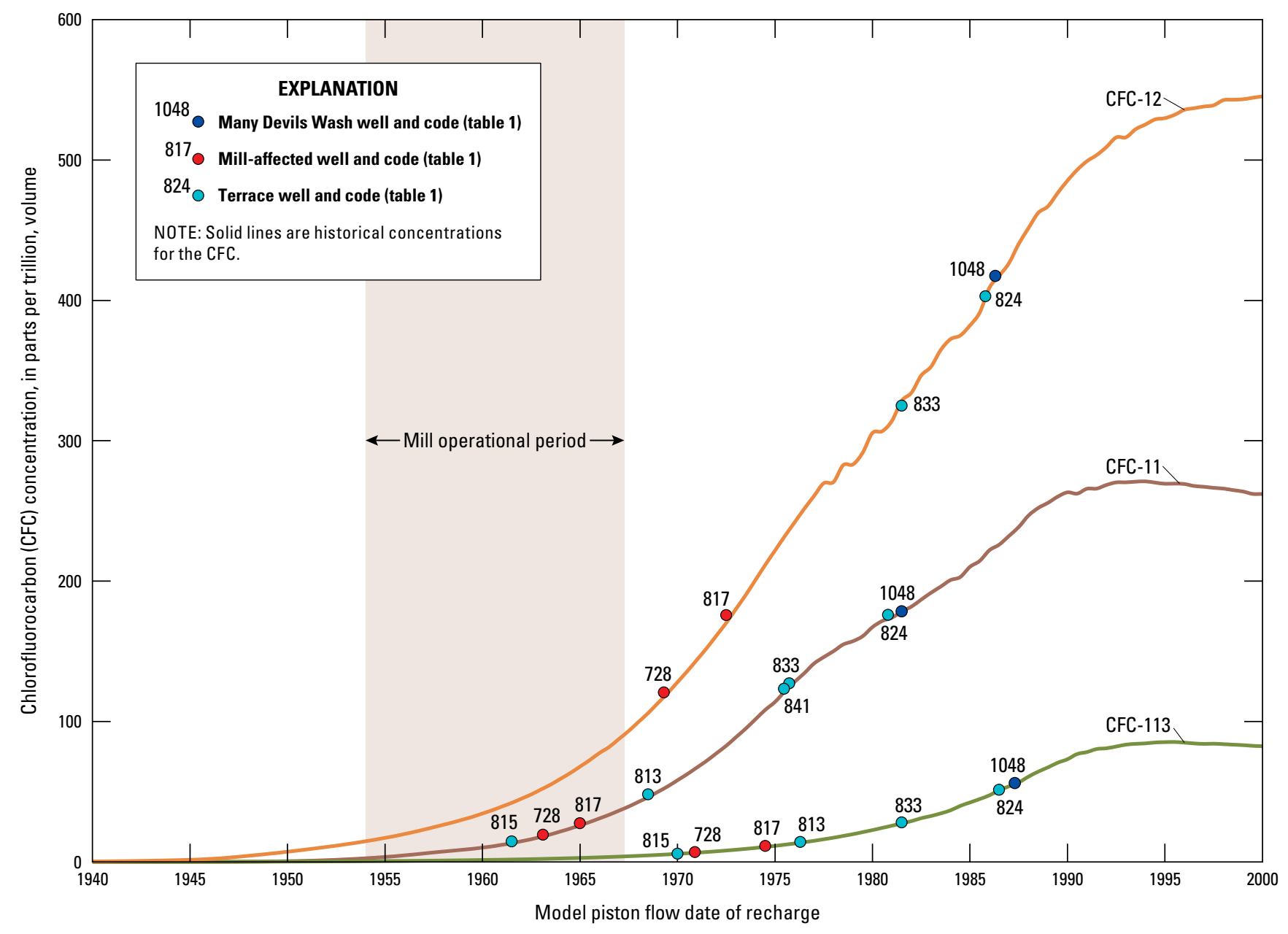

Figure 11. Chlorofluorocarbon (CFC) concentrations measured in groundwater from selected wells at the Shiprock Disposal Site, New Mexico, and historical concentrations of CFCs in the North American atmosphere. 
824 suggest that recharge occurred slightly later than the ${ }^{3} \mathrm{H}$ concentrations indicate. One important note is that well 824 is completed almost $180 \mathrm{ft}$ into the Mancos Shale. The presence of CFCs (and ${ }^{3} \mathrm{H}$ ) in well 824 indicates surficial recharge as the source of water in this well and not the upwelling of deep groundwater. The model dates (in the 1980s) for CFC concentrations in well 1048 suggest much younger water than indicated by the ${ }^{3} \mathrm{H}$. This discrepancy is thought to result from the shallow depth to water (approximately $5.5 \mathrm{ft}$ ) in the well. The shallow, unsaturated zone may allow for the exchange of present atmospheric CFC concentrations with the groundwater CFC concentrations (International Atomic Energy Agency, 2006).

Generally speaking, the apparent age of recharge from the measured CFC concentrations in the groundwater samples was younger than the estimated model dates based on the ${ }^{3} \mathrm{H}$ concentrations. This is likely because of the effects of evaporative fractionation that is discussed in the preceding section. The exception to this observation is in groundwater samples from Many Devils Wash, in which the CFC

concentrations suggest a much younger model date than the ${ }^{3} \mathrm{H}$ concentrations.

The CFC concentrations indicate that the groundwater sampled from most of the wells in the area of the study site recharged after the 1963 peak ${ }^{3} \mathrm{H}$ bomb pulse. In particular, CFC concentrations suggest that at least some wells on the terrace (for example, wells 813, 817, 824, and 833) have a substantial fraction of water that is composed of younger (postMill) water. Because of the potential for current atmospheric CFC concentrations to contribute to CFC concentrations in well 1048 , a reasonably certain age estimate from the CFC analysis cannot be provided for groundwater in Many Devils Wash.

\section{Source of Solutes in Many Devils Wash}

For this investigation, the dissolved constituents in the groundwater beneath Many Devils Wash are compared with constituent concentrations in groundwater samples from wells on the terrace, including those that are determined to be affected by Mill activities and from the analog sites. To aid in the interpretation of the groundwater chemical composition, a conceptual model of groundwater quality at the site is introduced. The possible sources of minor constituents, emphasizing the constituents of concern $\left(\mathrm{NO}_{3}, \mathrm{U}\right.$, and $\left.\mathrm{Se}\right)$, in the groundwater of Many Devils Wash are also examined.

\section{Conceptual Model of Water Quality at the Shiprock Disposal Site}

Much of the prior research on the dissolved constituents associated with the Mancos Shale has concluded that the degree of weathering results in significant geochemical changes to the mineral and organic content of the Mancos Shale, as well as to the groundwater that is in contact with it. The weathered
Mancos Shale is defined herein as parts of Mancos Shale that have undergone chemical changes that may be attributed to weathering processes, chiefly the oxidation of pyrite to iron hydroxide $(\mathrm{FeOOH})$ minerals and the oxidation and loss of organic matter (Morrison and others, 2012; Tuttle and others, 2014). These geochemical reactions manifest themselves in various observable ways at the site, including a change in the color of Mancos Shale from dark gray to yellowish gray and the production of a popcorn texture at the ground surface and tan-orange limonitic staining that typically occurs on bedding plane surfaces within the uppermost few feet of the Mancos Shale (U.S. Department of Energy, 2000; Morrison and others, 2012; Tuttle and others, 2014).

On the basis of an analysis of water extracts from samples of weathered and unweathered Mancos Shale collected from the West Salt Creek watershed near Grand Junction in western Colorado, Evangelou and others (1984) stated that the calcium-sulfate composition of groundwater in contact with the weathered Mancos Shale results from the dissolution of calcite, dolomite, and gypsum. The study found that there were substantially greater quantities of water-soluble ions in the partially weathered shale than in the unweathered shale, which was attributed to the presence of disseminated gypsum and calcite. Laronne (1977) also concluded that there were significantly greater quantities of water-soluble ions in the partially weathered shale than in the unweathered shale, which he attributed to the presence of gypsum.

A sodium-sulfate composition of groundwater in contact with the unweathered Mancos Shale was reported to result from the exchange of $\mathrm{Ca}$ for $\mathrm{Na}$ on the adsorption sites of clays found in the unweathered Mancos Shale (Evangelou and others, 1984). Evangelou and others (1984) suggest that the unweathered shale contains very little, if any, gypsum on the basis of equilibrium studies. It was later suggested that the most likely source of $\mathrm{Ca}$ in the unweathered Mancos Shale is from the dissolution of calcite (Tuttle and others, 2014). As divalent $\mathrm{Ca}$ ions are released into solution by the dissolution of calcite, they are exchanged for monovalent $\mathrm{Na}$ ions on the clay binding sites. In a modeling study of the Mancos Shale at Miller Creek, a field site in the Price River Basin, Utah, Azimi-Zonooz and Duffy (1993) supported early findings (Duffy and others, 1985) that the primary reactions leading the geochemical evolution of calcium-bicarbonate water to a sodium-sulfate water along the flow path were gypsum dissolution and cation exchange. Tuttle and Grauch (2009) and Tuttle and others (2014) have expanded on the weathering processes of the Mancos Shale to include the weathering of iron sulfide minerals that oxidize to form aqueous sulfates.

In order to gain an understanding of the site-specific mineralogy, a sample of the efflorescent crust, collected from the streambed of Many Devils Wash, two samples of loess collected in Many Devils Wash and in the East Fork, and surface samples of the Mancos Shale and the siltstone were collected with a hand trowel from two sites adjacent to the streambed of Many Devils Wash. Results of mineralogical analysis are presented in table 4 . 
Table 4. Mineralogy, in weight percentage, of selected solid samples collected from the Shiprock, New Mexico, area.

[Latitude and longitude are shown in parentheses]

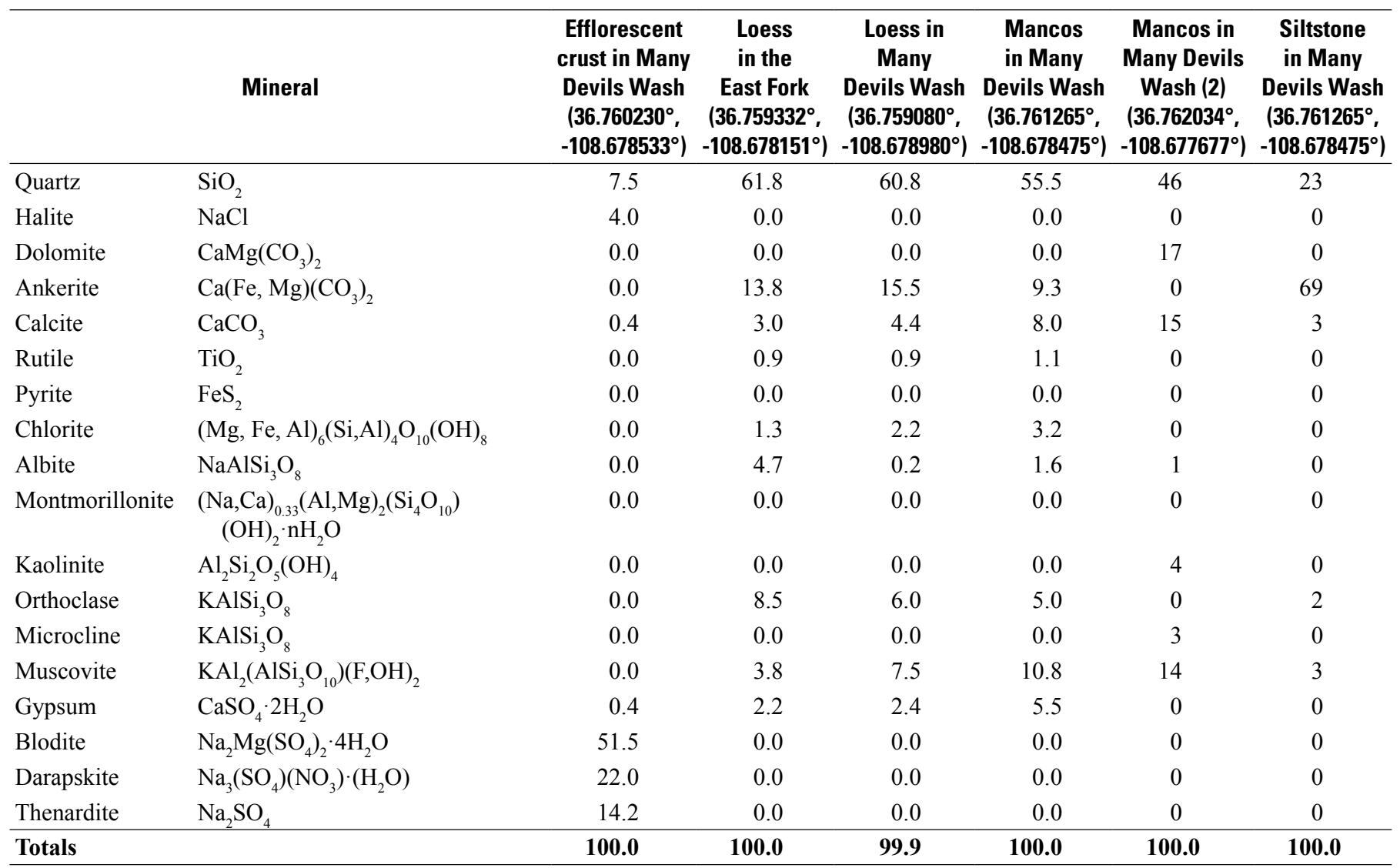

The large percentage of sodium salts (thernadite, darapskite, and blodite) left as efflorescent crusts when the groundwater evaporates upon reaching the surface, is an indication of the chemical makeup of the groundwater. The minerals in the efflorescent crust are also composed of sulfates (such as blodite and darapskite), which is consistent with other reported findings (Whittig and others, 1982), and the relatively small percentage of $\mathrm{NaCl}$ in the efflorescent crust sample suggests $\mathrm{Cl}$ does not compose a large part of the groundwater ion pool. The loess is primarily composed of resistant quartz; however, there is a substantial amount of aluminosilicate and carbonate minerals. Slight differences in the mineralogy of Mancos Shale samples may be the result of small differences in weathering, natural variability, and perhaps some incidental inclusion of loess in the sample. Unweathered samples of Mancos Shale were not collected or included in this analysis because the depths were inaccessible with available equipment.

With the exception of the thin ancestral San Juan River deposits, which consist primarily of resistive gravels and cobbles and quartz-dominated loess (table 4), the geology of the site is dominated by Mancos Shale. Given this geologic setting, the likely composition of groundwater is primarily from interactions between water and the minerals of the
Mancos Shale. The conceptual model of water quality at the site is based on the groundwater interactions with the Mancos Shale reported by the various studies referenced throughout this report, namely that the chemical composition of the water is dependent upon the amount of weathering the shale has undergone. If the shale is weathered, as defined herein, then a calcium-sulfate composition results from the dissolution of calcite, dolomite, and gypsum. In addition, the groundwater would generally be expected to be oxic because of the loss of oxidizable carbon in the weathering process. If the groundwater were in contact with the unweathered shale, the resulting composition would be sodium-sulfate, which results from the dissolution of calcite and the oxidation of pyrite, followed by cation exchange. The water associated with the unweathered shale would presumably be reducing because of the presence of oxidizable carbon. By extension, there are likely to be various degrees of weathering that would impart a composition in the groundwater sample that has components of each.

Weathered Mancos Shale is reported to overlie the unweathered Mancos Shale throughout the Shiprock Disposal Site. The weathered Mancos Shale is typically $5-10 \mathrm{ft}$ thick but may be as much as $30 \mathrm{ft}$ thick (U.S. Department of Energy, 2000); therefore, the water quality at the site may 
include groundwater compositions that reflect either types (weathered or unweathered) or some combination of the two. It is assumed that the majority of wells at the site were drilled to the first occurrence of water and are therefore completed within the alluvium and often into the weathering rind of the Mancos Shale. The well logs describe the Mancos Shale as having varying degrees of weathering. Of the wells selected for this investigation, three groundwater wells are described as being completed in the unweathered Mancos Shale: wells 824, 825, and 1059 (table 1).

\section{Major-Ion Chemistry}

The elements that make up the majority of solutes in natural waters include the cations $\mathrm{Ca}$, magnesium $(\mathrm{Mg}), \mathrm{Na}$, and potassium $(\mathrm{K})$ and the anions $\mathrm{SO}_{4}, \mathrm{Cl}$, carbonate $\left(\mathrm{CO}_{3}\right)$ and bicarbonate $\left(\mathrm{HCO}_{3}\right)$. Several reactions that may play an important role in imparting concentration differences of these solutes in groundwater associated with Mancos Shale include evapotranspiration and the precipitation and dissolution of minerals such as calcite, dolomite, gypsum, anhydrite, and halite. Other reactions include oxidation-reduction (redox) reactions, such as the oxidation of sulfide minerals that release $\mathrm{SO}_{4}$ to the water body, and cation exchange, which can preferentially adsorb divalent cations $(\mathrm{Ca}$ and $\mathrm{Mg}$ ) onto aluminosilicate clays and release monovalent cations such as $\mathrm{Na}$ and $\mathrm{K}$.

Examination of the major solutes in groundwater from the study area on the Piper diagram illustrates the overall prevalence of the anion $\mathrm{SO}_{4}$ and the varied distribution of cations between the monovalent $\mathrm{Na}$ ion and the divalent $\mathrm{Ca}$ and $\mathrm{Mg}$ ions (fig. 12). (Potassium is included in the analysis, but its percentage contribution to the total cations is negligible. In most wells, K contributes less than 1 percent. The maximum $\mathrm{K}$ contribution [3.3 percent] was measured in the samples from well 603.)

The Piper diagram is a trilinear diagram that is useful for illustrating the major-ion fractions of a water sample (Hem, 1985). The percentages on the axes of the diagram represent the relative abundances of ions in percent of milliequivalents per volume. In the analog and Many Devils Wash samples, $\mathrm{Na}$ accounted for more than 50 percent of the total cations in all the samples and more than 60 percent in all but one sample (the sample from well 1159, the most upgradient Many Devils Wash well); conversely, only one of the groundwater samples (from well 600) determined to be affected by Mill operations had a majority of the cation contribution from $\mathrm{Na}$. The 10 other Mill-affected wells were dominated by $\mathrm{Ca}, \mathrm{Mg}$, or a combination of the two. The difference in the cation fraction between the Mill-affected wells and the analog and Many Devils Wash wells may indicate that the shale underlying the disposal cell has undergone more weathering. (The weathering referred to here is the composite of the processes introduced above.) A higher degree of weathering could result from the large amounts of water and acidic raffinate that infiltrated into underlying aquifer after being discharged to the open-air ponds. The large amount of oxygenated water that was introduced is likely to have accelerated the geochemical reactions relative to the sites that were not being artificially recharged. In addition, the lower $\mathrm{pH}$ of the raffinate may have increased the dissolution of calcite, increasing the available $\mathrm{Ca}$ ions in the groundwater for cation exchange. Increased concentrations of $\mathrm{Ca}$ would, in time, reduce the number of clay mineral binding sites that have exchangeable $\mathrm{Na}$.

The terrace wells that were not classified as "Millaffected" include 11 wells that were sodium dominated and 10 that were $\mathrm{Ca}$ or $\mathrm{Mg}$ dominated. Sulfate accounted for the majority of anions in all but two of the wells. The groundwater samples from wells 824 and 825 had more $\mathrm{Cl}$ anions than $\mathrm{SO}_{4}$. The relatively low $\mathrm{Cl}$ content, compared with other anions throughout the site, are somewhat surprising given the marine origin of the Mancos Shale; however, this result is consistent with other findings (Evangelou and others, 1984; Laronne, 1977).

For the deeper aquifers at the site, Dam (1995) reports that the water types in the Dakota Sandstone were sodium bicarbonate and sodium sulfate, whereas water types in the Morrison aquifer were predominantly sodium bicarbonate in the recharge area, changing downgradient to sodium sulfate. Major ions in groundwater samples from well 648, screened in the Morrison aquifer, are dominated by $\mathrm{Na}_{\text {and }} \mathrm{SO}_{4}$ (fig. 12).

The sodium-sulfate composition of groundwater samples from the wells in Many Devils Wash, the analog sites, and many terrace wells suggests that there is unweathered (or partially unweathered) Mancos Shale in the area that is contributing $\mathrm{Na}$ to the groundwater through cation exchange. The distribution of cation percentages among wells also suggests that the amount of cation exchange is related to the amount of weathering. This is illustrated by the fact that the greatest $\mathrm{Na}$ contributions are associated with the wells that are described as unweathered in the lithologic descriptions (fig. 12). The cation distribution does appear to be an indicator of Mill affects, with the Mill-affected wells largely having a calcium/magnesium-sulfate composition that resembles the reported compositions of more weathered shale; however, that composition could change if the Mill-process water flowed into areas where the Mancos Shale was less weathered, according to the conceptual model of water quality.

\section{Geochemical Evolution}

Increases in the salinity (the amount of dissolved constituents in water) of waters associated with the Mancos Shale occur primarily from dissolved-solids concentrating processes, such as evaporation and transpiration, and by salt loading from the dissolution and weathering of residual salts in soil and geologic strata (Laronne and Schumm, 1977; Duffy, 1984). Table 5 shows the correlation between individual anions and cations and $\mathrm{SC}$ in each of the well categories, as determined by the coefficient of determination of a least squares regression and the $p$-value of a nonparametric Kendall rank correlation. (For this study, SC is regarded as a proxy for 


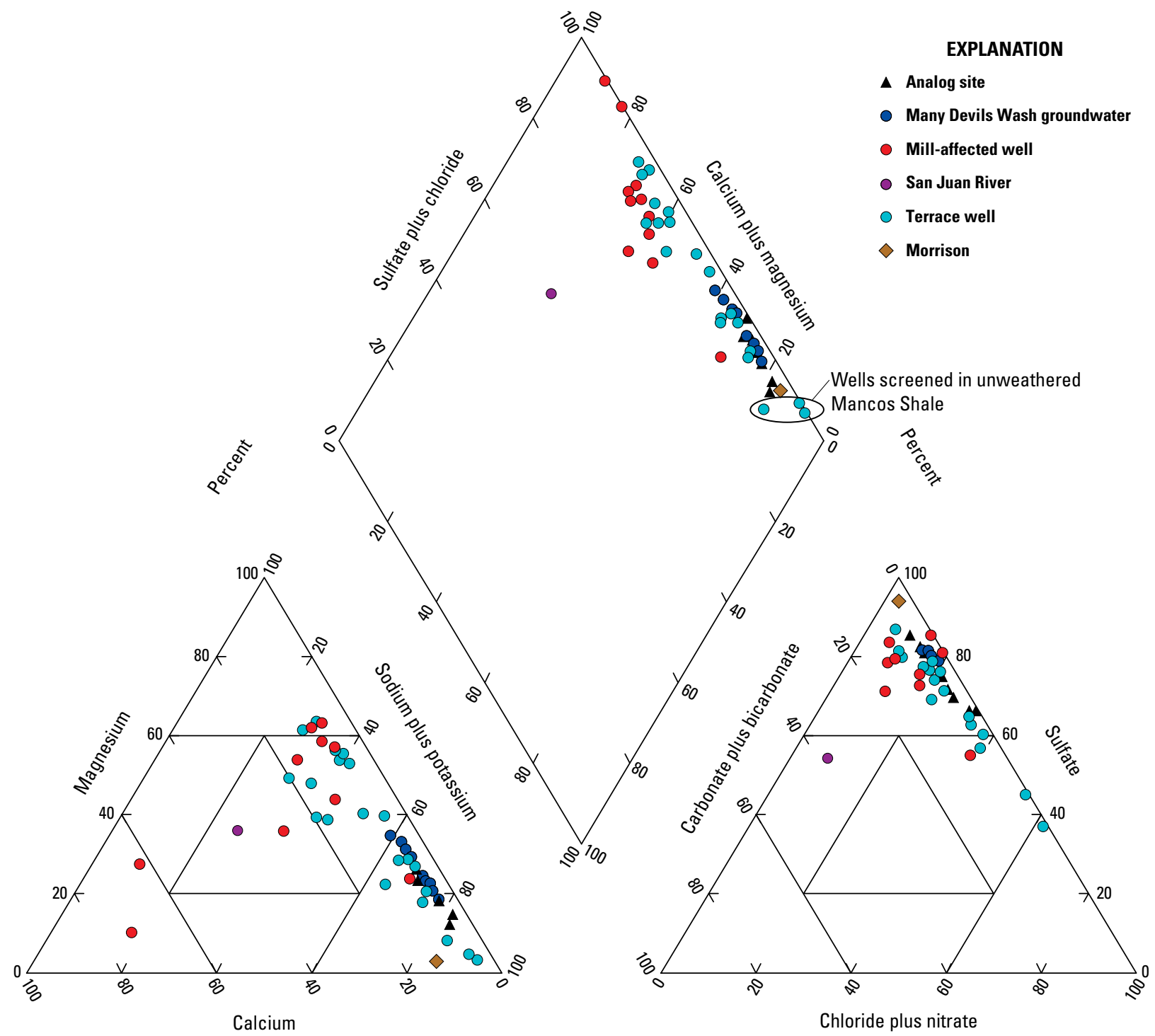

Percent of total ions, in milliequivalents per liter

Figure 12. Major-ion chemistry of groundwater samples at and near the Shiprock Disposal Site and surface-water samples from the San Juan River, New Mexico.

the concentration of dissolved solids.) The correlation between an ion or group of ions can help constrain geochemical processes affecting groundwater chemistry.

As shown in table 5, $\mathrm{SO}_{4}$ and $\mathrm{Na}$ have a linear, positive correlation with $\mathrm{SC}$ in all well groups. In contrast, $\mathrm{Cl}$ in the Many Devils Wash wells and the analog sites does not correlate particularly well with SC, suggesting that evapotranspiration is not the primary driver of increases in $\mathrm{SC}$ overall (although it might be important for certain wells). The positive correlation between $\mathrm{Na}$ and $\mathrm{SC}$, particularly in the analog wells, suggests that $\mathrm{Na}$ is tied to the weathering process. According to the conceptual model, the weathering process responsible for the increases in $\mathrm{Na}$ concentrations is cation exchange. Cation-exchange reactions are virtually instantaneous (Berkowitz, 2008), but on the aquifer level, the rate-limiting steps include the path the ion travels between aquifer solids, the weathering of minerals that contribute to the ion-exchange pool, and the number of mineral-binding sites with available cations; therefore, longer water-rock interactions would yield greater concentrations of $\mathrm{Na}$.

In order to test the geochemical validity of the proposed flow paths introduced in the "Hydraulic Gradients" section, the dissolved constituents in groundwater from wells along Many Devils Wash and the wells between the disposal cell and Many Devils Wash, the East Mill flow path ( $A-A^{\prime}$ ' in fig. 4$)$, were compared by using the conceptual model for water 
Table 5. Coefficient of determination for the least squares regression and $p$-values of ions to specific conductivities.

$\left[\mathrm{R}^{2}\right.$, coefficient of determination; SC, specific conductivity]

\begin{tabular}{|c|c|c|c|c|c|c|c|c|c|c|}
\hline & \multicolumn{2}{|c|}{ All sites } & \multicolumn{2}{|c|}{$\begin{array}{l}\text { Terrace } \\
\text { wells }\end{array}$} & \multicolumn{2}{|c|}{$\begin{array}{l}\text { Mill-affected } \\
\text { wells }\end{array}$} & \multicolumn{2}{|c|}{$\begin{array}{l}\text { Many Devils } \\
\text { Wash wells }\end{array}$} & \multicolumn{2}{|c|}{ Analog sites } \\
\hline & $\mathbf{R}^{2}$ & $p$-value & $\mathbf{R}^{2}$ & $p$-value & $\mathbf{R}^{2}$ & $p$-value & $\mathbf{R}^{2}$ & $p$-value & $\mathbf{R}^{2}$ & $p$-value \\
\hline \multicolumn{11}{|c|}{ Anions } \\
\hline SC:Sulfate & 0.80 & $2.2 \mathrm{E}-16$ & 0.47 & $8.1 \mathrm{E}-04$ & 0.87 & $6.1 \mathrm{E}-04$ & 0.76 & $5.3 \mathrm{E}-04$ & 0.76 & 0.01 \\
\hline SC:Chloride & 0.41 & $2.2 \mathrm{E}-16$ & 0.33 & $1.2 \mathrm{E}-05$ & 0.78 & $3.1 \mathrm{E}-03$ & 0.15 & 0.62 & 0.44 & 0.06 \\
\hline SC:Nitrate & 0.10 & 0.01 & 0.26 & 0.02 & 0.16 & 0.53 & 0.56 & $1.1 \mathrm{E}-03$ & 0.57 & 0.02 \\
\hline SC:Bicarbonate & 0.05 & 0.07 & 0.03 & 0.20 & 0.87 & $1.8 \mathrm{E}-03$ & 0.48 & 0.01 & 0.33 & 0.08 \\
\hline \multicolumn{11}{|c|}{ Cations } \\
\hline SC:Sodium & 0.88 & $2.2 \mathrm{E}-16$ & 0.70 & $5.1 \mathrm{E}-06$ & 0.73 & $2.0 \mathrm{E}-02$ & 0.74 & $3.2 \mathrm{E}-05$ & 0.91 & 0.01 \\
\hline SC:Calcium & 0.02 & 0.35 & 0.04 & 0.74 & 0.11 & 0.21 & $1.2 \mathrm{E}-03$ & 0.69 & 0.10 & 0.62 \\
\hline SC:Potassium & $1.0 \mathrm{E}-03$ & 0.07 & 0.26 & 0.01 & 0.60 & $3.1 \mathrm{E}-03$ & 0.02 & 0.66 & 0.71 & 0.02 \\
\hline SC:Magnesium & 0.10 & 0.07 & 0.17 & 0.15 & 0.82 & $3.1 \mathrm{E}-03$ & 0.23 & 0.01 & 0.40 & 0.39 \\
\hline
\end{tabular}

quality at the site. Based on these concepts introduced above, $\mathrm{Na}$ may be an indicator of the relative position of a well along a flow path. In order to examine the geochemical evolution along the proposed flow paths, $\mathrm{Na}$ and $\mathrm{Cl}$ concentrations were plotted together (fig. 13).

The plot of Na concentrations in the Many Devils Wash wells (fig. 13) generally shows increasing $\mathrm{Na}$ concentrations from the upper watershed (well 1159) down to the knickpoint and the area where groundwater seeps begin to appear (well 1049). Based on the geochemical processes presented above and the relation between $\mathrm{Na}$ and $\mathrm{SC}$, an increase in $\mathrm{Na}$ along a flow path may indicate longer water-rock interactions (in this case by cation exchange following the dissolution of calcite and (or) gypsum).

When $\mathrm{Na}$ concentrations are plotted with $\mathrm{Cl}$ concentrations, it is clear that $\mathrm{Cl}$ concentrations do not increase with Na concentrations ( $p$-value $=0.92)$ in the groundwater beneath Many Devils Wash as one would expect from either evapotranspiration or the dissolution of halite (greater mass gains in $\mathrm{Cl}$ than $\mathrm{Na}$ ). A theoretical evaporation line (a 1:1 $\mathrm{Na}: \mathrm{Cl}$ molar equivalent line) is displayed to show the expected concentration changes from well 1159 if evaporation was taking place. Because the relation is based on an equal number of $\mathrm{Na}$ and $\mathrm{Cl}$ ions, the line also represents the expected change if halite dissolution was occurring or if dilution, from a substantially lower dissolved-solid water (such as precipitation), was to occur. Based on the reported lack of leachable $\mathrm{Cl}$ from the Mancos Shale, increasing waterrock interactions (in other words, groundwater evolution down a flow path) would not yield an increase in $\mathrm{Cl}$. The small differences in $\mathrm{Cl}$ concentrations in the wells along
Many Devils Wash may be the result of various influxes of atmospheric $\mathrm{Cl}$ though recharge at distinct points and unique evaporation concentration factors. The relation between $\mathrm{Na}$ and $\mathrm{Cl}$ in Many Devils Wash suggests a geochemical evolution that is in agreement with the conceptual model and therefore supports the existence of a groundwater flow path down the axis of the channel.

In the wells between the disposal cell and Many Devils Wash, $\mathrm{Na}$ also increases with distance from the disposal cell; however, the $\mathrm{Cl}$ concentrations measured in groundwater samples from well 1058 suggest a disconnected flow path between the disposal cell and Many Devils Wash. As noted in the "Hydraulic Gradients" section, well 1058 (fig. 8) exhibits some groundwater-elevation fluctuations that do not trend with other nearby wells and therefore may not be along the same flow path as wells 830, 603, 731, and 1059. The consistent rise in $\mathrm{Na}$ and $\mathrm{Cl}$ from the disposal cell to Many Devils Wash, without well 1058, may reflect a realistic geochemical evolution, although without an identifiable geologic source of $\mathrm{Cl}$.

It is also important to note that the wells in the lower part of the Many Devils Wash watershed do not fall along a mixing line between groundwater in the wells between the disposal cell and Many Devils Wash and groundwater from the upper watershed wells. Although a possible relation between these two ions can still be considered between the disposal cell and Many Devils Wash, the relation of $\mathrm{Na}$ and $\mathrm{Cl}$ along the proposed flow path down Many Devils Wash supports other evidence that groundwater in Many Devils Wash originates as precipitation in the watershed and undergoes a predictable geochemical evolution as it flows downgradient. 


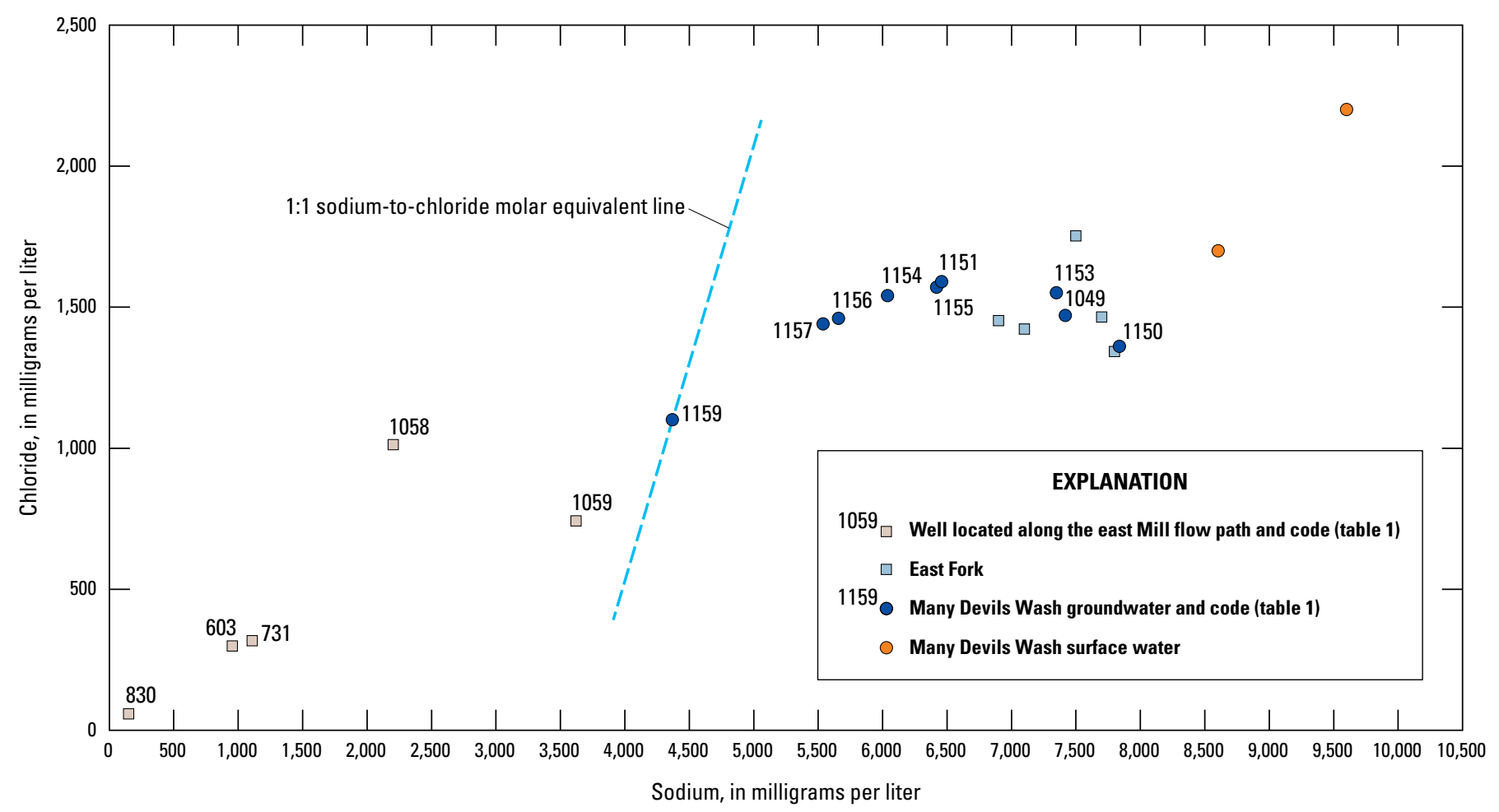

Figure 13. Sodium- and chloride-ion concentrations in select groundwater and surface-water samples at and near the Shiprock Disposal Site, New Mexico.

Sulfate also correlates with SC in most wells at the site, suggesting that sulfur is tied to the weathering processes (table 5). According to Tuttle and Grauch (2009), at the onset of weathering in the Mancos Shale, iron-sulfide minerals oxidize to create $\mathrm{SO}_{4}$, which can later form gypsum with a unique sulfur isotopic composition (delta sulfur-34 of sulfate $\left.\left[\delta^{34} \mathrm{~S}_{\text {sulfate }}\right]\right)$ that is relatively light $\left(\delta^{34} \mathrm{~S}_{\text {sulfate }}\right.$ values below $\left.-20 \%\right)$ compared with the sulfur that has not been reduced in the past. Tuttle and Grauch (2009) proposed that these isotopes could be used to identify the sources of salts in the Colorado River on the basis of this fractionation. The sulfur isotopes of $\mathrm{SO}_{4}$ reported in the groundwater at the site (U.S. Department of Energy, 2012a) are discussed in this report to help understand the potential weathering processes occurring at the site.

In groundwater, the sulfur $\left(\delta^{34} \mathrm{~S}_{\text {sulfate }}\right)$ and oxygen $\left(\delta^{18} \mathrm{O}_{\text {sulfate }}\right)$ isotopic compositions in $\mathrm{SO}_{4}$ result from the composition of the source elements and from the isotopic fractionation that subsequently occurs as a result of chemical and biological processes. Mayer (2005) provides an overview and a list of references for studies investigating the sources of $\mathrm{SO}_{4}$ in hydrology by isotopic composition. (The analysis in this study is restricted to a discussion of $\delta^{34} \mathrm{~S}_{\text {sulfate }}$ in $\mathrm{SO}_{4}$, not $\delta^{18} \mathrm{O}_{\text {sulfate }}$ in $\mathrm{SO}_{4}$, because the former is more relevant to the processes investigated herein.)
Diagenetic sulfide minerals associated with marine sediments are depleted in ${ }^{34} \mathrm{~S}$ compared to marine $\mathrm{SO}_{4}$ and commonly have $\delta^{34} \mathrm{~S}$ values averaging -22\%o (Tuttle and others, 2014). Because there is little fractionation during the oxidation of pyrite, the sulfur ( $\mathrm{S}$ ) in the $\mathrm{SO}_{4}$ formed by the oxidation of pyrite retains the lighter sulfur composition (similarly negative $\delta^{34} \mathrm{~S}$ values). The majority of $\delta^{34} \mathrm{~S}_{\text {sulfate }}$ values of groundwater samples are near or below $-20 \%$ $(-19.46$ to $-31.14 \%$ o $)$ at the Shiprock Disposal Site and in the analog wells as reported by the U.S. Department of Energy (2012a). The exception to the depleted $\delta^{34} S_{\text {sulfate }}$ values that are commonly observed in the groundwater around the site are the relatively enriched $\delta^{34} \mathrm{~S}_{\text {sulfate }}$ values $(-4.75-0.99 \%$ ) of groundwater samples taken from four of the wells determined to be affected by the Mill (wells 817, 826, 1007, and 1074). Because sulfuric acid was used in ore processing at most uranium mills, the $\delta^{34} \mathrm{~S}_{\text {sulfate }}$ can provide a unique isotopic fingerprint of the groundwater derived from Mill recharge. Sulfuric acid is usually produced from hydrogen sulfide gas $\left(\mathrm{H}_{2} \mathrm{~S}\right)$ recovered from oil and gas production (The Sulfur Institute, 2014). Faure (1986) states that the $\delta^{34} \mathrm{~S}$ values of the $\mathrm{S}$ in $\mathrm{H}_{2} \mathrm{~S}$ gas range from -8 to $+32 \%$, significantly more enriched than marine sulfides. 


\section{The Source of Groundwater and Solutes to Many Devils Wash at a Former Uranium Mill Site in Shiprock, New Mexico}

Several investigations have reported $\delta^{34} \mathrm{~S}_{\text {sulfate }}$ values in water samples from wells associated with uranium-milling activity. Hurst and Solomon (2008) determined the $\delta^{34} \mathrm{~S}_{\text {sulfate }}$ values in water samples collected from three tailings cells and 16 monitoring wells at the White Mesa Uranium Mill in Blanding, Utah. The values of $\delta^{34} \mathrm{~S}_{\text {sulfate }}$ from the tailings cells fell within the reported range for sulfur derived from sour gas and cluster close to $0 \%$ (range from $-1.04 \%$ to $-0.89 \%$ ). The $\delta^{34} \mathrm{~S}_{\text {sulfate }}$ values in the monitoring wells ranged from -3.0\%o to $13.6 \%$, with all but two wells being enriched $\left(\delta^{34} \mathrm{~S}_{\text {sulfate }}>\right.$ $5.0 \%$ ). The authors do not propose an explanation for the enriched $\delta^{34} \mathrm{~S}_{\text {sulfate }}$ values but do suggest that the difference in the $\delta^{34} \mathrm{~S}_{\text {sulfate }}$ values between the wells and the tailings cells supports other evidence that the cells are not leaking. In 1981, Ries (1982) collected water samples to determine the sulfurisotope composition in $\mathrm{SO}_{4}$ from monitoring wells, mine discharge, and mill tailings ponds located in the Ambrosia Lake uranium mining district in west-central New Mexico. The study concluded that the $\mathrm{SO}_{4}$ associated with the mill, mines, and underlying aquifer had different origins. A sample of the sulfuric acid used at one of the mills had a $\delta^{34} \mathrm{~S}_{\text {sulfate }}$ value of 5.8\% (Ries, 1982). The sulfur used in the production of the acid was reported to be supplied from $\mathrm{H}_{2} \mathrm{~S}$ recovered in gas production. The $\mathrm{SO}_{4}$ in water samples collected from two tailings ponds had $\delta^{34} \mathrm{~S}_{\text {sulfate }}$ values of -1.1 and $3.7 \%$. The enriched $\delta^{34} \mathrm{~S}_{\text {sulfate }}$ values (range from -1.9 to $6.0 \%$ ) in samples from five wells near the Mill ponds were used to indicate the extent that the pond leachate had migrated.

The sulfur isotopes in sulfate support the earlier classification system for Mill-affected wells and are distinct enough in groundwater from the analog sites and the Many Devils Wash wells to suggest a different source for $\mathrm{SO}_{4}$ than the groundwater from Mill-affected wells. Because only four wells near the disposal cell were analyzed for $\mathrm{SO}_{4}$ isotopes, sulfur isotopes were not used to determine whether a well has been affected by the Mill.

The light sulfur composition (negative $\delta^{34} \mathrm{~S}_{\text {sulfate }}$ values) of $\mathrm{SO}_{4}$ in the groundwater samples from many of the wells near the site (table 1) provides evidence for the oxidation of pyrite in the Mancos Shale being the origin of $\mathrm{SO}_{4}$. The depleted $\delta^{34} S_{\text {sulfate }}$ values in the background wells are similar to values reported by Tuttle and others (2007) for gypsum in Mancos Shale soil samples collected from the Gunnison Gorge National Conservation Area in southwestern Colorado $\left(\delta^{34} \mathrm{~S}_{\text {sulfate }}\right.$ values ranged from 0.6 to $-26.0 \%$, with 16 of the 17 samples ranging from -15.2 to $-26.0 \%$.). The negative $\delta^{34} \mathrm{~S}_{\text {sulfate }}$ values for $\mathrm{SO}_{4}$ isotopes reported for the site not only provide evidence for the origin of $\mathrm{SO}_{4}$ but also that the gypsum found in the Mancos Shale at the site is formed in-situ. As $\mathrm{O}_{2}$ and water infiltrate into the Mancos Shale, the pyrite is oxidized, releasing $\mathrm{SO}_{4}$ into the groundwater. As groundwater conditions change, this $\mathrm{SO}_{4}$ may precipitate as gypsum, which may later be redissolved as water infiltrates or the water table increases. Because there is no substantial fractionation in $\mathrm{SO}_{4}$ isotopes during the precipitation or dissolution of gypsum, it cannot be determined whether the
$\mathrm{SO}_{4}$ measured in the groundwater results directly from pyrite oxidation or through the dissolution of gypsum.

The conceptual model for the major-ion chemistry is reflected in the concentrations in groundwater measured at the site. A calcium-sulfate groundwater composition results from the dissolution of calcite, dolomite, and gypsum when in contact with weathered Mancos Shale. In the unweathered Mancos Shale, $\mathrm{SO}_{4}$ ultimately derived from the oxidation of pyrite (with or without subsequent formation and dissolution of gypsum) and $\mathrm{Ca}$ ions from the dissolution of calcite are released into solution. Through ion exchange, Ca replaces $\mathrm{Na}$ on the exchange sites of clay minerals resulting in water with a sodium-sulfate composition. As Ca is adsorbed to clay binding sites, it becomes unavailable to bind with other anions, such as $\mathrm{SO}_{4}$.

\section{Minor Constituents}

The elevated concentrations of constituents of concern measured in groundwater beneath Many Devils Wash led to the inference that these constituents were derived from the Mill (U.S. Department of Energy, 2000); as a result, DOE accepted responsibility for remediation of the arroyo. The evidence presented in the previous sections of this report, however, does not support the conclusion that Mill water is or has affected groundwater beneath Many Devils Wash; therefore, alternate explanations for the origin of elevated concentrations of $\mathrm{NO}_{3}, \mathrm{U}$, and $\mathrm{Se}$ in the groundwater beneath Many Devils Wash must be identified. This section explores whether or not the origin of these constituents could be the local geology and whether or not a natural origin can be distinguished from an anthropogenic source.

\section{Nitrate}

Possible sources of $\mathrm{NO}_{3}$ to Many Devils Wash include the ammonia used by the Mill to precipitate the U, geologic nitrogen from the underlying Mancos Shale, and the soil $\mathrm{NO}_{3}$ pool accumulated in the unsaturated zone through atmospheric deposition. Nitrogen is generally not regarded as a conservative species in soil and groundwater, participating in numerous biological and chemical processes. Much of the work pertaining to nitrates in the Mancos Shale largely focuses on agricultural $\mathrm{NO}_{3}$ (McMahon and others, 1999; Wright, 1999). More recently, a few studies have examined the contributions of geologic nitrogen. Holloway and Dahlgren (2002) summarized work on general geologic nitrogen sourcing, and that work was followed by a study of nitrogen in the Mancos Shale (Holloway and Smith, 2005). A site investigation compared $\mathrm{NO}_{3}$ concentrations and isotopes in groundwater samples from around the Shiprock Disposal Site (Garvin, 2012).

Nitrogen mineralization is the process by which nitrogen bound to sedimentary organic matter or living things is converted to inorganic forms of nitrogen such as ammonium 
$\left(\mathrm{NH}_{4}^{+}\right)$(McMahon and others, 1999). In oxic environments, mineralization is followed by nitrification, in which $\mathrm{NH}_{4}{ }^{+}$is oxidized to nitrite $\left(\mathrm{NO}_{2}^{-}\right)$and then to $\mathrm{NO}_{3}^{-}$. Nitrification may also reduce the $\mathrm{pH}$ of the system with the production hydrogen ions $\left(\mathrm{H}^{+}\right)$(and water $\left[\mathrm{H}_{2} \mathrm{O}\right]$ ) $(\mathrm{Hem}, 1985)$. Both $\mathrm{NH}_{4}{ }^{+}$and $\mathrm{NO}_{3}$ may be utilized by plants and animals. These reactions are summarized by the following equations:

$$
\begin{gathered}
\text { Mineralization: organic nitrogen } \rightarrow \text { ammonium }\left(\mathrm{NH}_{4}^{+}\right) \\
\text {Nitrification: } \\
2 \mathrm{NH}_{4}^{+}+3 \mathrm{O}_{2} \leftrightarrow \mathrm{NO}_{2}^{-}+4 \mathrm{H}^{+}+2 \mathrm{H}_{2} \mathrm{O} \\
2 \mathrm{NO}_{2}^{-}+\mathrm{O}_{2} \leftrightarrow 2 \mathrm{NO}_{3}^{-}
\end{gathered}
$$

Denitrification is the microbially mediated process in which $\mathrm{NO}_{3}$ is reduced to molecular nitrogen in the absence of oxygen (Holloway and Smith, 2005). Examples of denitrification reactions that may occur with two electron donors that have been identified in the Mancos Shale (reduced sulfur $\left[\mathrm{FeS}_{2}\right]$ and carbon $[\mathrm{C}]$ ) are summarized by the following equations:

$$
\begin{gathered}
2 \mathrm{FeS}_{2}+6 \mathrm{NO}_{3}^{-}+2 \mathrm{H}_{2} \mathrm{O} \leftrightarrow 3 \mathrm{~N}_{2}+4 \mathrm{SO}_{4}^{2-}+2 \mathrm{FeOOH}+2 \mathrm{H}^{+} \\
5 \mathrm{C}+4 \mathrm{NO}_{3}^{-}+2 \mathrm{H}_{2} \mathrm{O} \leftrightarrow \mathrm{CO}_{2}+2 \mathrm{~N}_{2}+4 \mathrm{HCO}_{3}^{-}
\end{gathered}
$$

Geologic nitrogen may exist in sedimentary rocks as relict organic matter or from the substitution of ammonium for $\mathrm{K}$ in sedimentary, igneous, and metamorphic rock (Lowe and Wallace, 2001). Holloway and Dahlgren (2002) reported that nitrogen originates in sedimentary rocks as kerogen. During early digenesis, new labile organic matter is produced in the sediments. The nitrogen is partially liberated from this labile organic matter as $\mathrm{NH}_{4}^{+}$and is incorporated into clay minerals. In the oil shale of the Green River Formation, between 41 and 84 percent of the nitrogen measured in five samples was found to be present as ammonium fixed within silicate minerals (Cooper, 1983). Cooper proposed that the $\mathrm{N}$ was derived from organic matter in the highly reducing bottom waters that generated substantial quantities of $\mathrm{NH}_{3}$ that was then converted to $\mathrm{NH}_{4}^{+}$during digenesis. Power and others (1974) found appreciable quantities of exchangeable $\mathrm{NH}_{4}{ }^{+}$in the organic-rich sedimentary rocks of the Fort Union Formation. They also determined that a lack of oxygen at depth prohibits nitrification, but when the units were exposed to atmospheric weathering, nitrification proceeds, resulting in increased concentrations of $\mathrm{NO}_{3}$ and decreased concentrations of $\mathrm{NH}_{4}^{+}$.

Holloway and Smith (2005) measured 1,020-1,270 milligrams per kilograms of nitrogen in samples from a Mancos Shale outcrop in the Grand Valley in Colorado; 58-74 percent of the nitrogen was organic, and the remaining fraction was estimated to be a combination of ammonium sorbed to clay mineral surfaces, ammonium substituted into clay and mica-group minerals, and $\mathrm{NO}_{3}$ salts. Despite the high nitrogen content in the soils, the natural background concentrations of nitrogen in surface water and groundwater were surprisingly low, which Holloway and Smith (2005) speculate may be because of microbial denitrification processes enhanced by the organic carbon in the bedrock. Ammonia volatilization may also be a significant process for nitrogen loss where the $\mathrm{pH}$ is greater than 8 (Holloway and Smith, 2005).

Nitrate concentrations measured in the wells along the proposed flow paths of groundwater to Many Devils Wash are plotted with $\mathrm{Na}$ concentrations in figure 14. The increasing concentrations of $\mathrm{Na}$ along the Many Devils Wash flow path, presented in the "Conceptual Model of Water Quality at the Shiprock Disposal Site" section, were interpreted to be consistent with increasing water-rock interactions along the flow path. When illustrated graphically, $\mathrm{Na}$ and $\mathrm{NO}_{3}$ concentrations generally increase linearly $(p$-value $=1.1$ $\left.\left[10^{-4}\right]\right)$, suggesting that both may be resulting from groundwater interactions with the Mancos Shale. This relation also indicates that the $\mathrm{NO}_{3}$ is not solely sourced from atmospheric deposition. Although not conservative like $\mathrm{Cl}$, groundwater concentrations of $\mathrm{NO}_{3}$ would not be expected to increase along the flow path (similar to $\mathrm{Cl}$ in fig. 13) if the source of $\mathrm{NO}_{3}$ were atmospheric deposition. Evaporation is also not considered to be responsible for the coinciding increases in the concentrations of $\mathrm{Na}$ and $\mathrm{NO}_{3}$.

Nitrate concentrations are exceedingly high in the groundwater beneath Many Devils Wash (862-3,181 mg/L), as well as in the groundwater at the analog sites (601-2,338 $\mathrm{mg} / \mathrm{L})$, the terrace wells $(12-8,786 \mathrm{mg} / \mathrm{L})$, and the Millaffected wells $(86-5,529 \mathrm{mg} / \mathrm{L})$ (table 1). Garvin (2012) applied the "2-to-1 rule" to $\mathrm{NO}_{3}$ isotopes collected from groundwater beneath Many Devils Wash to suggest that $\mathrm{NO}_{3}$ was sourced by the local nitrification of ammonium. The "2-to-1 rule" suggests that during nitrification of ammonium, two of the oxygen atoms in $\mathrm{NO}_{3}{ }^{-}$are derived from the groundwater and one oxygen atom is derived from the air. By using the $\delta^{18} \mathrm{O}$ values measured in the groundwater and atmosphere, an estimated $\delta^{18} \mathrm{O}$ for $\mathrm{NO}_{3}$ may be calculated. This work was expanded upon by DOE who found similar results in other samples collected in the area (U.S. Department of Energy, 2012a). This work does suggest a plausible source for $\mathrm{NO}_{3}$ from geologic ammonium; however, the analysis could not definitively differentiate between geologic and anthropogenic sources of $\mathrm{NO}_{3}$.

The oxidizing conditions that are required for nitrification must also be preserved in the groundwater to prevent denitrification and maintain the extremely high concentrations of $\mathrm{NO}_{3}$. Aerobic conditions are seemingly present based on some of the dissolved- oxygen concentrations reported in the DOE GEMS database and this investigation. The common presence of aerobic conditions is somewhat unexpected given the reported high carbon concentration of unweathered shale. The high $\mathrm{NO}_{3}$ concentrations in the groundwater beneath Many Devils Wash suggest that the oxidation of carbon and sulfide may be more or less complete while there is still cation exchange occurring. 


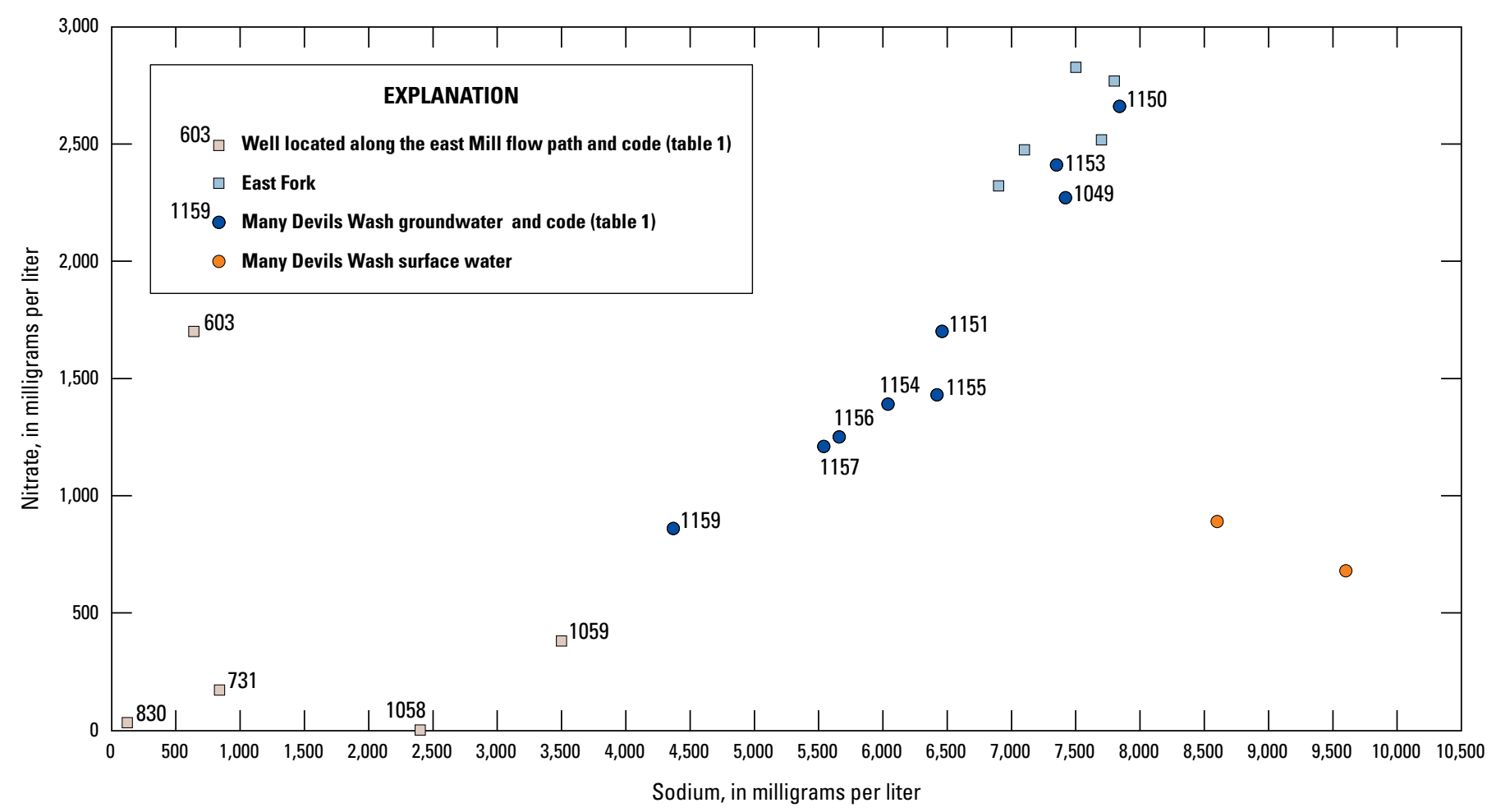

Figure 14. Sodium and nitrate ion concentrations in select Shiprock, New Mexico, groundwater and surface-water samples.

\section{Uranium}

Evidence presented by previous investigations (Public Health Service, 1962; U.S. Department of Energy, 2000) and as part of this study indicates that high $U$ concentrations measured in the groundwater of many wells at the site are undoubtedly caused by operations at the Mill; however, $\mathrm{U}$ concentrations are also high in groundwater from the analog sites (table 1). As presented in the "Groundwater Well Classification" section of this report, the U alpha ARs differ substantially between groundwater measured in the Mill-affected wells and those measured at the analog sites, suggesting a natural source of $U$ in the area. Uranium has been reported to be naturally occurring in the Mancos Shale, primarily from the ash-fall deposits that fell into the Cretaceous sea from regional volcanism (Pliler and Adams, 1962; U.S. Department of Energy, 2011b). The $\mathrm{U}$ concentrations in the solid phase are fairly consistent throughout the geographic distribution of the Mancos Shale. Pliler and Adams (1962) collected samples of the Mancos Shale from 16 sites in Colorado, Utah, Arizona, and New Mexico. The average $U$ concentration in 102 samples was 3.7 parts per million (ppm) and ranged from 0.9 to $12 \mathrm{ppm}$; 70 percent of the values were between 2 and $4 \mathrm{ppm}$. Of the 102 samples, 10 samples were collected 14 mi west of
Shiprock, N. Mex. The average U concentration in these samples was $3.9 \mathrm{ppm}$ and ranged from 1.5 to $5.5 \mathrm{ppm}$. Tuttle and others (2007) also reported small variations in whole-rock concentrations from Mancos Shale and soil samples from the Gunnison Gorge National Conservation Area in southwestern Colorado and Hanksville, Utah. The average concentration for the 528 samples was $4.5 \mathrm{ppm}$ (median was $4.4 \mathrm{ppm}$ ), with most (77 percent) of the samples having concentrations between 3 and 5 ppm (Tuttle and others, 2007).

After deposition, $\mathrm{U}$ was likely to have been incorporated into the sediments on the sea bottom. Subsequent release to the groundwater can occur through oxidative weathering. In general, the mobility of $U$ in groundwater is dependent upon its redox state and the environmental conditions that influence its ability to form complexes. Uranium in its oxidized hexavalent (VI) state is at least 10,000 times more soluble than in its reduced tetravalent state (IV) (Sherman and others, 2007). Desorption of $U$ from the matrix material is further enhanced by increases in alkalinity and $\mathrm{pH}$ (Langmuir, 1978). In groundwater with high carbonate concentrations, $\mathrm{U}$ complexes stay in solution and do not easily adsorb to negatively charged mineral surfaces (Abdelouas and others, 1998).

The $\mathrm{U}$ concentrations of groundwater from all sites not affected by the Mill have a relatively smaller range of $U$ 
concentrations $(0.0002-0.55 \mathrm{mg} / \mathrm{L})$ than the concentrations measured in the Mill-affected wells $(0.0006-10.5 \mathrm{mg} / \mathrm{L})$. Much of the variability in wells that are not designated as Mill-affected occurs in the terrace wells, which may be partially influenced by the Mill (fig. 15).

The median $\mathrm{U}$ concentrations in the groundwater from Many Devils Wash, the analog sites, and the terrace wells are greater than the median concentrations measured in water extracts of the soil and shale samples reported by Tuttle and others (2007) and the median concentrations reported for seeps and springs at several sites around the Colorado Plateau (U.S. Department of Energy, 2011b). The greatest median U concentration was calculated for groundwater samples collected in wells that have been determined to be affected by the Mill (fig. 15).

The relatively small amount of variability in the $\mathrm{U}$ concentrations in groundwater at the site that has not been affected by the Mill may be because of the uniform distribution of $U$ in the Mancos Shale; however, the $U$ concentrations in extracts from the sample sites reported by Tuttle and others (2007) ranged dramatically from less than
$0.0001 \mathrm{mg} / \mathrm{L}$ to $0.35 \mathrm{mg} / \mathrm{L}$ (70 percent were below $0.01 \mathrm{mg} / \mathrm{L}$ ). Tuttle and others (2007) do not offer an explanation for this, and no relations are obvious from the data presented. These results may suggest that the aqueous concentrations of $U$ in groundwater are influenced not only by the overall $\mathrm{U}$ concentrations of the host rock but also by the mineral phases in which they are found. The higher ARs (over about 2 ) in groundwater from the analog seeps and the Many Devils Wash wells, as well as the low ${ }^{3} \mathrm{H}$ values, suggest relatively long residence times of the groundwater that could allow for an averaging of $U$ concentrations and the dependent aqueous species, resulting in small differences in the groundwater concentrations. These small variations are striking and may suggest a solubility control for $U$ in the groundwater beneath Many Devils Wash. In contrast, the introduction of different ores, oxidizing agents, and water volumes at the Mill site, over a relatively small period of time, may have created highly variable conditions in the groundwater. The dissolution, dilution, and complexing that would yield $U$ concentrations that vary greatly in both time and space are exhibited in the Mill-affected wells.

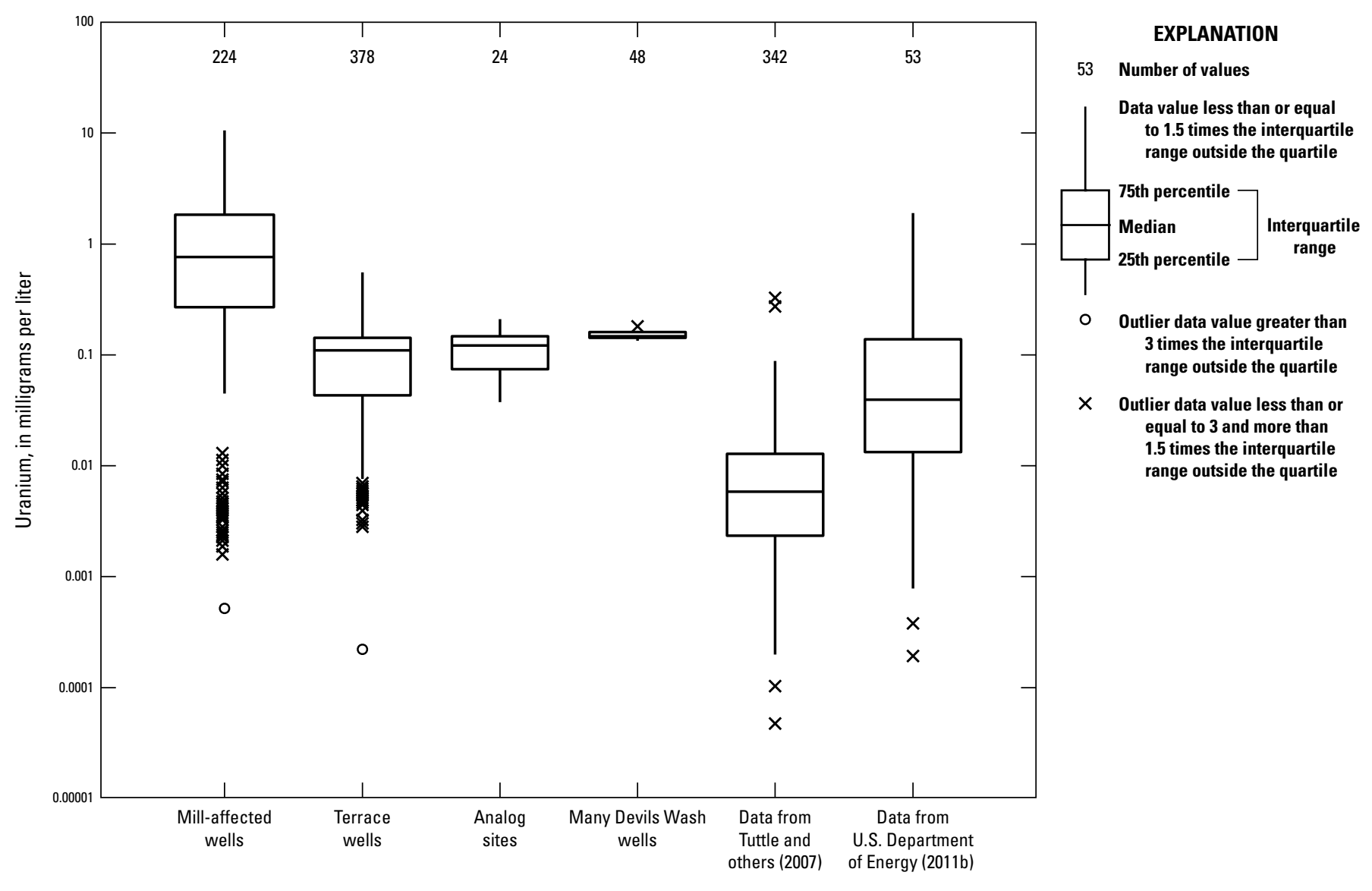

Figure 15. Uranium (U) concentrations in groundwater samples at and near the Shiprock Disposal Site, Shiprock, New Mexico, regional Mancos Shale seeps reported by the U.S. Department of Energy (2011b), and soil and shale extracts from Mancos Shale reported by Tuttle and others (2007). 
Based on the widespread presence of $U$ in the Mancos Shale and the distribution of aqueous $U$ in the analog sites and other sites in the region, it appears likely that $U$ in the groundwater of Many Devils Wash is naturally sourced from the Mancos Shale. The conclusions reached in this section are supported by the ARs measured around the site.

\section{Selenium}

Selenium has been documented by multiple studies to be present in waters associated with the Mancos Shale (Butler and others, 1996; Wright, 1999; Thomas and others, 2008; Mast and others, 2014). Selenium occurrence in the Cretaceous deposits is likely to be related to volcanic sources that ultimately deposited in the Cretaceous sea (Butler and others, 1996). The organic-rich, reducing environment coprecipitated Se with isomorphous sulfur in sulfide-bearing minerals such as pyrite (Butler and others, 1996). Selenium in unweathered shale resides in pyrite, rare clausthalite, and possibly organic matter (Mast and others, 2014), but most is thought to be incorporated with diagenetic pyrite (Tuttle and others, 2014). Oxidation converts primary, reduced Se to selenate, which is released to the groundwater or stored as a component of $\mathrm{SO}_{4}$ salts (Mast and others, 2014; Tuttle and others, 2014). The distribution of Se concentrations in groundwater at and near the site, as well as from two other studies, is presented in figure 16 .

The average Se concentrations in groundwater from the analog sites and Many Devils Wash are substantially greater than concentrations measured in groundwater from the Millaffected wells, terrace wells, concentrations reported in the extracts from Tuttle and others (2007), and the groundwater seeps from the U.S. Department of Energy (2011b). In the "Major-Ion Chemistry" section of this report, the possibility of greater weathering of the shale under the disposal cell was introduced to help explain the associated groundwater chemistry. Tuttle and others (2009) suggested that the amount of Se that can be extracted from the host rock by water decreases with increasing weathering, and that the metal is labile during early weathering and is continually lost from the weathering profile. Lower Se concentrations in groundwater from the Mill-affected wells, relative to the Many Devils Wash wells and analog sites, also would be consistent with the idea

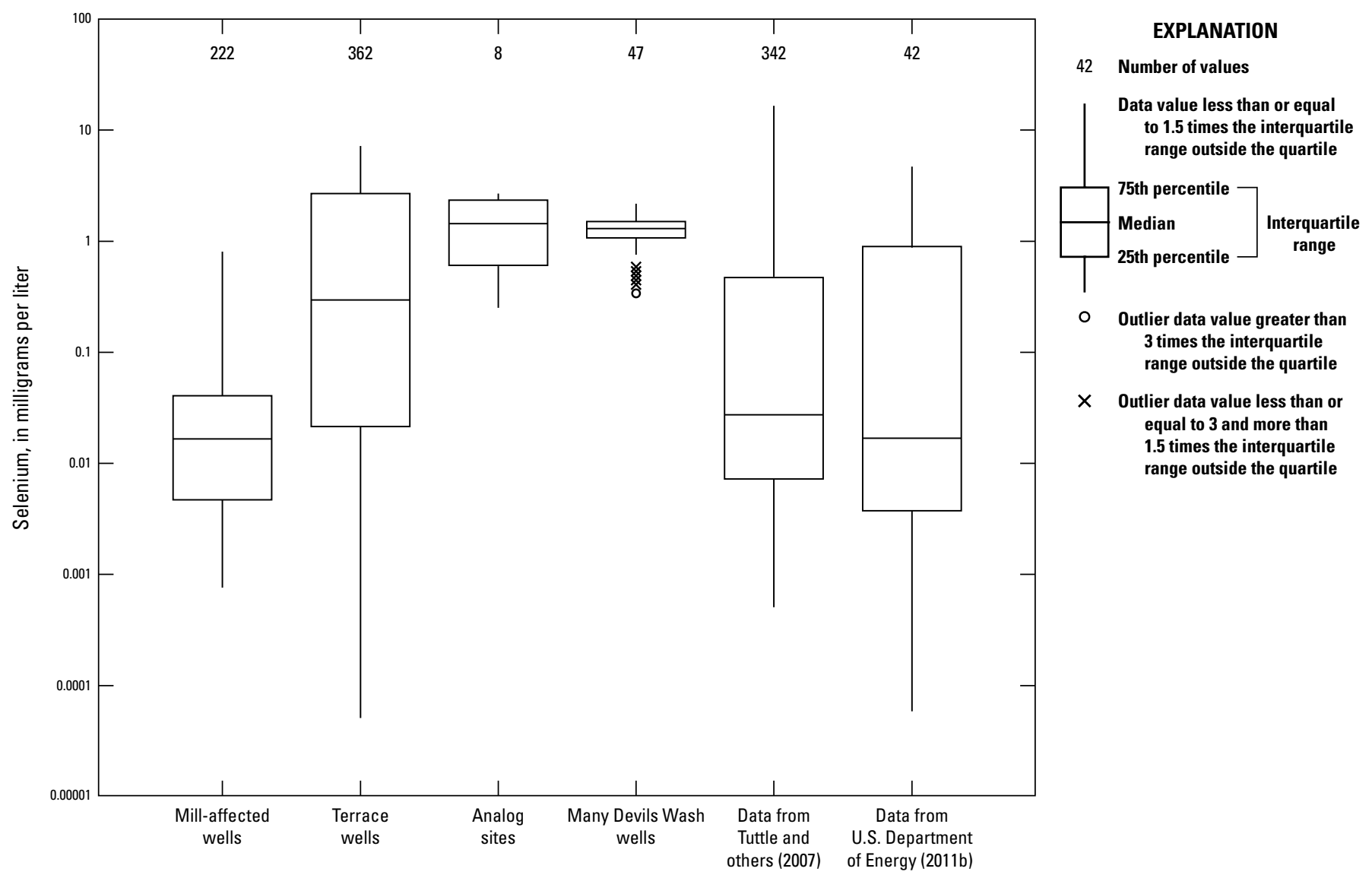

Figure 16. Selenium concentrations in groundwater samples at and near the Shiprock Disposal Site, Shiprock, New Mexico, regional Mancos Shale seeps reported by the U.S. Department of Energy (2011b), and soil and shale extracts from the Mancos Shale reported by Tuttle and others (2007). 
that more weathering has occurred beneath the disposal cell. The large variability in Se concentrations in the groundwater from other terrace wells relative to other wells in the area of the Shiprock Disposal Site also supports earlier interpretations that groundwater in the terrace wells beyond the cell may have been influenced to varying degrees by the Mill operations.

The documented occurrence of Se in waters associated with the Mancos Shale is evidence of a natural origin for the constituent in the groundwater beneath Many Devils Wash. The oxidation of sulfides, primarily pyrite, is thought to also release Se into the groundwater as selenate, and it may be stored in the $\mathrm{SO}_{4}$ salts.

The range and distribution of concentrations for $\mathrm{NO}_{3}, \mathrm{U}$, and Se in Many Devils Wash are consistent with a geological origin for the solutes. The findings in the "Minor Constituents" section support the conceptual model of water quality at the Shiprock Disposal Site.

\section{Summary}

The Shiprock Disposal Site is the location of the former Navajo Mill, a uranium ore-processing facility that was in operation from 1954 to 1968 . The site is located on a terrace overlooking the San Juan River in the town of Shiprock, New Mexico. Following closure of the Mill, all tailings and associated materials were encapsulated in a disposal cell built on top of the existing tailings piles. The milling operations conducted at the site created radioactive tailings and processrelated wastes that are now found in the groundwater. Elevated concentrations of constituents of concern - ammonium, manganese, nitrate, selenium, strontium, sulfate, and uranium - have also been measured in groundwater seeps in the nearby Many Devils Wash arroyo, leading to the inference that these constituents were derived from the Mill. Some or all of these constituents have also been reported to originate from the underlying Mancos Shale bedrock. The purpose of this report was to increase the understanding of the source of water and solutes to the groundwater beneath Many Devils Wash and to establish the background concentrations for groundwater that is in contact with the Mancos Shale at the site. This report presents evidence on three working hypotheses: (1) the water and solutes in Many Devils Wash originated from the operations at the former Mill, (2) groundwater in deep aquifers is upwelling under artesian pressure to recharge the shallow groundwater of Many Devils Wash, and (3) the groundwater beneath Many Devils Wash originates as precipitation that infiltrates into the shallow aquifer system and discharges to Many Devils Wash in a series of springs on the east side of the wash. The solute concentrations in the shallow groundwater of Many Devils Wash would result from the interaction of the water and the Mancos Shale if the source of water was upwelling from deep aquifers or precipitation.

In order to address the purpose of this report, it is necessary to understand and compare the features of background, or natural, groundwater quality relative to groundwater that has been affected by Mill activities. The analog water-quality data were collected by the U.S. Department of Energy from groundwater seeps and springs in the Upper Eagle Nest Arroyo and Salt Creek Wash, located north of the San Juan River, making them hydraulically removed from the Mill site and related activities. Groundwater wells were classified as affected by the Mill using the reported uranium alpha activity ratios (AR) measured in groundwater samples, along with the uranium (U) concentrations and the well locations relative to the Mill. Activity ratios equal to or less than 1.2 were determined to be the most reliable indicator of Mill-affected groundwater. Wells that had a reported AR of 1.2 or less were classified as Mill affected.

Because of the large quantities of water used in the milling process, the Mill is considered a significant source of water to the terrace and likely had effects on the groundwater beyond the site itself. That process water created a groundwater mound under the Mill that migrated outward in the unconsolidated deposits along the top of bedrock surface. On the basis of the available groundwater-elevation data from 2012, a hydraulic gradient allowing for the movement of groundwater from the disposal cell to Many Devils Wash presently does not exist. This lack of potential is supported by the observation that groundwater seeps in Many Devils Wash occur on the bank opposite from the disposal cell.

Discharge from underlying deep aquifers has been suggested as a possible source of water to the shallow aquifer through vertical leakage or the preferential flow through fractures or joints; however, the Mancos Shale is described as a confining layer for those units, and there is approximately 218 feet (ft) of Mancos Shale between the alluvium and the Gallup Sandstone and another $685 \mathrm{ft}$ of the lower Mancos Shale to the Dakota Sandstone. Although artesian flow in well 648 demonstrates an upward groundwater gradient that would allow for the possibility of upward flow, several wells completed into the Mancos Shale around the site are either dry or have a downward gradient. Additional evidence that the shallow groundwater is not being recharged by the deep aquifers is the stable isotope composition of water. The stable isotopes (delta deuterium $[\delta \mathrm{D}]$ and delta oxygen-18 $\left[\delta^{18} \mathrm{O}\right]$ ) of water in all the shallow groundwater samples at the site were enriched relative to the Global Meteoric Line, and many were also enriched relative to the Arid Meteoric Line, suggesting evaporative losses. Most groundwater samples from the Many Devils Wash were slightly more enriched in $\delta \mathrm{D}$ than from the Mill-affected groundwater samples, but the difference is small and could not confidently be distinguished. All wells, however, are substantially more enriched in both $\delta^{18} \mathrm{O}$ and $\delta \mathrm{D}$ than groundwater samples from the deeper aquifers, indicating that substantial upwelling is unlikely.

Another potential source of groundwater recharge to Many Devils Wash is the focused recharge of precipitation. Several observations indicate that focused recharge is the likely source of groundwater in the area of Many Devils Wash. The visible erosional features in Many Devils Wash provide evidence of piping, a process by which precipitation moves 
through the unsaturated zone, and groundwater sapping, a process of erosion at the base of arroyo walls by groundwater emergence. The distribution and type of vegetation in Many Devils Wash suggest the accumulation of groundwater beneath the channel, but there are no consistent phreatophytes or wetland vegetation that might reveal a constant upwelling through a fracture. Groundwater elevations measured in wells along Many Devils Wash indicate that the hydraulic gradient would allow for groundwater from precipitation falling in the upper reaches to accumulate and flow down the axis of the channel toward the seeps. The estimated recharge from precipitation was calculated to be 0.0008 inches per year (in/yr) by using the mass-balance approach from the reported seep discharge and $0.0011 \mathrm{in} / \mathrm{yr}$ by using the chloride $(\mathrm{Cl})$ mass-balance approach. Because only one approach includes a precipitation term, the similar results suggest that precipitation is a plausible and likely source of recharge to Many Devils Wash.

Tritium $\left({ }^{3} \mathrm{H}\right)$ concentrations above 19 tritium units (in 2012-13) measured in groundwater samples from wells on the terrace provide evidence that Mill-age water exists in the shallow groundwater on the terrace. The ${ }^{3} \mathrm{H}$ concentrations in wells between the disposal cell and Many Devils Wash support other evidence that water is no longer moving into Many Devils Wash. The concentrations and distribution of ${ }^{3} \mathrm{H}$ in groundwater beneath Many Devils Wash do not appear to be consistent with the Mill being the source of groundwater. Despite the uncertainties associated with chlorofluorocarbon (CFC) concentrations measured in the groundwater samples, the CFC concentrations help to constrain the ${ }^{3} \mathrm{H}$ model dates. The CFC concentrations suggest that the apparent age of most of the groundwater on the terrace is younger than 1963, and much of it is likely to have recharged following the closure of the Mill.

With the exception of the thin ancestral San Juan River deposits, which consist primarily of resistive gravels and cobbles and quartz-dominated loess, the geology of the site is dominated by Mancos Shale. Given this geologic setting, the likely composition of groundwater results primarily from interactions between water and the minerals of the Mancos Shale. The conceptual model of water quality at the site is based on the groundwater interactions with the Mancos Shale reported by the various studies referenced throughout this report, namely that the chemical composition of the water is dependent upon the amount of weathering the shale has undergone. If the shale is weathered, as defined herein, then a calcium-sulfate composition results from the dissolution of calcite, dolomite, and gypsum. In addition, the groundwater would generally be expected to be oxic because of the loss of oxidizable carbon in the weathering process. If the groundwater were in contact with the unweathered shale, the resulting composition would be sodium-sulfate, which results from the dissolution of calcite and the oxidation of pyrite, followed by cation exchange. Weathered Mancos Shale is reported to overlie the unweathered Mancos Shale throughout the Shiprock Disposal Site; therefore, the water quality at the site may include groundwater compositions that reflect either types or some combination of the two.

In the analog and Many Devils Wash groundwater samples, Na accounted for more than 50 percent of the total cations in all the samples and more than 60 percent in all but one (the sample from the most upgradient Many Devils Wash well, well 1159). Conversely, only one of the groundwater samples from a well affected by Mill operations (well 600) had a majority of the cation contributions from Na. The seven other Mill-affected wells were dominated by calcium, magnesium, or the combination of the two. The remaining terrace wells included 11 wells that were sodium $(\mathrm{Na})$ dominated and 10 that were calcium-magnesium dominated. Sulfate accounted for the majority of anions in all but two of the wells.

The sodium-sulfate composition of groundwater samples from the wells in Many Devils Wash, the analog sites, and many terrace wells suggests that there is unweathered Mancos Shale in the area that is contributing $\mathrm{Na}$ to the groundwater through cation exchange. The distribution of cation percentages among wells also suggests that the amount of cation exchange is related to the amount of weathering. This is illustrated by the fact that the greatest $\mathrm{Na}$ contributions are associated with the wells where the geology was described as unweathered Mancos Shale. The cation distribution does appear to be an indicator of Mill effects, with the Millaffected wells largely having a calcium/magnesium-sulfate composition that resembles the reported compositions of more weathered shale; however, that composition could change if the Mill process water flowed into areas where the Mancos Shale was less weathered.

The positive correlation between $\mathrm{Na}$ and specific conductivity, particularly in the analog wells, suggests that $\mathrm{Na}$ is tied to the weathering process. The relation of $\mathrm{Na}$ and $\mathrm{Cl}$ in Many Devils Wash suggests a geochemical evolution from cation exchange and supports the existence of a groundwater flow path down the axis of the channel. Sulfate also correlates well with specific conductivity in most wells at the site, suggesting that sulfur is tied to the weathering processes. The majority of $\delta^{34} \mathrm{~S}_{\text {sulfate }}$ groundwater results at the site and in the analog wells are near or below -20 per mil, supporting the concept that sulfate is formed in-situ from the oxidation of sulfide found in the Mancos Shale.

Nitrate $\left(\mathrm{NO}_{3}\right)$ concentrations are exceedingly high in the groundwater beneath Many Devils Wash. Sodium and $\mathrm{NO}_{3}$ concentrations generally increase down Many Devils Wash, suggesting that higher $\mathrm{NO}_{3}$ may be caused by groundwater interactions with the Mancos Shale. High concentrations of $\mathrm{NO}_{3}$ in groundwater across the area (including at the analog sites) and the reported high nitrogen content of Mancos Shale support the conclusion that the elevated $\mathrm{NO}_{3}$ concentrations in the groundwater beneath Many Devils Wash is likely to result from natural processes.

The $\mathrm{U}$ measured in groundwater from all the sites not affected by the Mill have a relatively small range of concentrations and exhibit far less variability than the 
concentrations measured in the groundwater of Mill-affected wells. On the basis of the widespread presence of $U$ in the Mancos Shale and the distribution of aqueous $U$ in the analog sites and other sites in the region, it appears likely that $U$ in the groundwater of Many Devils Wash is naturally sourced from the Mancos Shale. The natural source of $U$ in the Many Devils Wash is supported by the uranium ARs measured around the site.

The abundance of studies documenting selenium (Se) associated with the occurrence of Mancos Shale provides evidence for the Mancos Shale being a likely source for the constituent. The average Se concentrations in groundwater from the analog sites and Many Devils Wash are substantially greater than the concentrations measured in groundwater from the Mill-affected wells and terrace wells, which supports the possibility of greater weathering of the shale under the disposal cell. The oxidation of sulfides, primarily pyrite, is thought to also release Se into the groundwater as selenate, and it may be stored in the sulfate salts.

On the basis of the multiple lines of evidence presented in this report, the source of water to the groundwater beneath Many Devils Wash is likely the focused recharge of precipitation. The amount of recharge is estimated to be small given the large potential evaporative losses and low annual precipitation rates. The precipitation falling in the Many Devils Wash watershed would collect in the channels and topographic low points, infiltrate into the alluvium, and then flow north along the erosional bedrock contacts to and along the axis of the channel. As the groundwater flows north along the axis of the Many Devils Wash, it interacts with the weathered and unweathered Mancos Shale. The geochemical evolution down the flow path results in the sodium-sulfate composition of the groundwater and is the source of several constituents of concern.

\section{References Cited}

Abdelouas, A., Lutze, W., and Nuttall, E., 1998, Chemical reactions of uranium in ground water at a mill tailings site: Journal of Contaminant Hydrology, v. 34, no. 4, p. 343-361.

Aeschbach-Hertig, W., Peeters, F., Beyerle, U., and Kipfer, R., 1999, Interpretation of dissolved atmospheric noble gases in natural waters: Water Resources Research, v. 35, p. 2779-2792.

Albrethsen, Holger, Jr., and McGinley, F.E., 1982, Summary history of domestic uranium procurement under U.S. Atomic Energy Commission contracts, final report: U.S. Department of Energy Report GJBX-220, 14 p.

Allison, C.D., and Ashcroft, Nick, 2014, New Mexico Range Plants: Circular 374, accessed on various dates, at http:// aces.nmsu.edu/pubs/_circulars/CR374_SM.pdf.
Allison, G.B., 1988, A review of some of the physical, chemical and isotopic techniques available for estimating groundwater recharge: NATO ASI Series, v. 222, p. 49-72.

Anderholm, S.K., 1994, Ground-water recharge near Santa Fe, north-central New Mexico: U.S. Geological Survey WaterResources Investigations Report 94-4078, 68 p.

Azimi-Zonooz, Ali, and Duffy, C.J., 1993, Modeling transport of subsurface salinity from a Mancos Shale hillslope: Groundwater, v. 31, no. 6, 972 p.

Ball, B.A., Cobban, W.A., Merewether, E.A., Grauch, R.I., McKinney, K.C., and Livo, K.E., 2010, Fossils, lithologies, and geophysical logs of the Mancos Shale from core hole USGS CL-1 in Montrose County, Colorado: U.S. Geological Survey Open-File Report 2009-1294, 38 p.

Bartolino, J.R., 1997, Chlorofluorocarbon and tritium age determination of ground-water recharge in the Ryan Flat subbasin, Trans-Pecos Texas: U.S. Geological Survey Water-Resources Investigations Report 96-4245, 29 p.

Bayer, R., Schlosser, P., Bonisch, G., Rupp, H., Zaucker, F., and Zimmek, G., 1989, Performance and blank components of a mass spectrometric system for routine measurement of helium isotopes and tritium by the $3 \mathrm{He}$ in-growth method: Sitzungsberichte der Heidelberger Akademie der Wissenschaften, Mathematisch-naturwissenschaftliche Klass, Jahrgang 1989, Springer-Verlag, 42 p.

Berkowitz, B., Dror, I., and Yaron, B., 2008, Contaminant geochemistry-Interactions and transport in the subsurface environment: Berlin, Springer, 412 p.

Blanchard, P.J., Roy, R.R., and O'Brien, T.F., 1993, Reconnaissance investigation of water quality, bottom sediment, and biota associated with irrigation drainage in the San Juan River area, San Juan County, northwestern New Mexico, 1990-91: U.S. Geological Survey WaterResources Investigations Report 93-4065, 141 p.

Bolin, B., 1958, On the use of tritium as a tracer for water in nature: Proceedings 2nd International Conference on the Peaceful Uses of Atomic Energy, p. 336-344.

Breecker, D.O., Sharp, Z.D., and McFadden, L.D., 2009, Seasonal bias in the formation and stable isotopic composition of pedogenic carbonate in modern soils from central New Mexico, USA: Geological Society of America bulletin, v. 121, no. 3-4, p. 630-640.

Brister, B.S., and Hoffman, G.K., 2002, Fundamental geology of San Juan Basin energy resources, in Brister, B.S., and Price, L.G., eds., New Mexico's energy, present and futureDecision-makers field conference 2002: The New Mexico Bureau of Geology, p. 21-25. 
Butler, D.L., Wright, W.G., Stewart, K.C., Osmundson, B.C., Krueger, R.P., and Crabtree, D.W., 1996, Detailed study of selenium and other constituents in water, bottom sediment, soil, alfalfa, and biota associated with irrigation drainage in the Uncompahgre project area and in the Grand Valley, west-central Colorado, 1991-93: U.S. Geological Survey Water-Resources Investigations Report 96-4138, 136 p.

Chapelle, F.H. and Knobel, L.L., 1985, Stable carbon isotopes of $\mathrm{HCO}_{3}{ }^{-}$in the Aquia aquifer, Maryland-Evidence for an isotopically heavy source of $\mathrm{CO}_{2}$ : Groundwater, v. 23, no. 5, p. 592-599.

Clark, I.D., and Fritz, P., 1997, Environmental isotopes in hydrogeology: Boca Raton, Fla., CRC Press/Lewis Publishers, $328 \mathrm{p}$.

Clarke, W.B., Jenkins, W.J., and Top, Z., 1976, Determination of tritium by mass spectrometric measurement of $3 \mathrm{He}$ : International Journal of Applied Radiation and Isotopes, v. 27 , p. 515-522.

Cook, P.G., Plummer, L.N., Solomon, D.K., Busenberg, E., and Han, L.F., 2006, Effects and processes that can modify apparent CFC age, in Use of chlorofluorocarbons in hydrology - A guidebook: Vienna, Austria, International Atomic Energy Agency, p. 31-58.

Cooper, J.E., 1983, Ammonium-nitrogen in Green River Formation oil shale: Science v. 219, no. 4584, p. 492-493.

Coplen, T.B., Herezeg, A.L., and Barnes, C., 2000, Isotope engineering-using stable isotopes of the water molecule to solve practical problems, in Cook, P.G., and Herezeg, A.L., eds., Environmental tracers in subsurface hydrology: Boston, Kluwer Academic Publishers, p. 79-110.

Craig, H., 1961, Isotopic variations in meteoric waters: Science, v. 133, no. 3465, p. 1702.

Craigg, S.D., 2001, Geologic framework of the San Juan structural basin of New Mexico, Colorado, Arizona, and Utah, with emphasis on Triassic through Tertiary rocks: U.S. Geological Survey Professional Paper 1420, p. 63.

Dam, W.L., 1995, Geochemistry of ground water in the Gallup, Dakota, and Morrison aquifers, San Juan Basin, New Mexico: U.S. Geological Survey Water-Resources Investigations Report 94-4253 p. 76.

Duffy, C.J., 1984, Conceptual models of geologic and agricultural salt loads in streams of the upper Colorado River Basin in Salinity in Watercourses and Reservoirs: Butterworth Publishers, Mass., p. 223-233.

Duffy, C.J., Jurinak, J.J., Sangani, S., and Azimi, A., 1985, Identification and modeling the impact of marine shale bedrock on groundwater and stream salinity-Upper Colorado River Basin: Utah Water Research Laboratory, Utah State University, 68 p.
Evangelou, V.P., Whittig, L.D., and Tanji, K.K., 1984, Dissolved mineral salts derived from Mancos Shale: Journal of Environmental Quality, v. 13, no. 1, p. 146-150.

Faure, Gunter, 1986, Principles of isotope geology: New York, N.Y., John Wiley \& Sons, 589 p.

Friedman, I., Smith, G.I., Gleason, J.D., Warden, A., and Harris, J.M., 1992, Stable isotope composition of waters in southeastern California-modern precipitation: Journal of Geophysical Research, v. 97, no. d5, p. 5795-5812.

Garvin, P.F., 2012, A stable isotope investigation of NO3 contamination at Many Devils Wash, Shiprock UMTRCA site, Shiprock, NM: Reno, University of Nevada, Master's Thesis, $92 \mathrm{p}$.

Genereux, D.P., and Hooper, R.P., 1998, Oxygen and hydrogen isotopes in rainfall-runoff studies, in Kendall, C., and McDonnell, J.J., eds., Isotope tracers in catchment hydrology: Amsterdam, Elsevier Science B.V., p. 319-346.

Hall, S.A., 1990, Holocene landscapes of the San Juan Basin, New Mexico; geomorphic, climatic, and cultural dynamics, in Lasca, N.P., and Donahue, J., eds., Archaeological geology of North America: Boulder, Colo., p. 323-334.

Helsel, D.R., and Hirsch, R.M., 2002, Statistical methods in water resources: U.S. Geological Survey Techniques of Water Resources Investigations, book 4, chap. A3, 522 p.

Hem, J.D., 1985, Study and interpretation of the chemical characteristics of natural waters, $3 \mathrm{~d}$ ed.: U.S. Geological Survey Water-Supply Paper 2254, 263 p.

Holloway, J.M., and Dahlgren, R.A., 2002, Nitrogen in rockOccurrences and biogeochemical implications: Global Biogeochemical Cycles, v. 16, no. 4, p. 65-61-65-17.

Holloway, J.M., and Smith, R.L., 2005, Nitrogen and carbon flow from rock to water-Regulation through soil biogeochemical processes, Mokelumne River watershed, California, and Grand Valley, Colorado: Journal of Geophysical Research, v. 110, no. F01010, p. 12.

Hurst, T.G., and Solomon, D.K., 2008, Summary of work completed, data results, interpretations and recommendations for the July 2007 sampling event at the Denison Mines, USA, White Mesa Uranium Mill near Blanding: Report prepared for the Utah Division of Radiation Control, 62 p.

Ingraham, N.L., 1998, Isotopic variations in precipitation, in Kendall, C., and McDonnell, J.J., eds., Isotope tracers in catchment hydrology: Amsterdam, Elsevier Science B.V., p. 87-118.

International Atomic Energy Agency, 2006, Use of chlorofluorocarbons in hydrology, a guidebook: Vienna, Austria, International Atomic Energy Agency, 291 p. 
Jurgens, B.C., Böhlke, J.K., and Eberts, S.M., 2012, TracerLPM (Version 1)-An Excel $\AA$ workbook for interpreting groundwater age distributions from environmental tracer data: U.S. Geological Survey Techniques and Methods Report 4-F3, 60 p.

Kendall, Carol, and Coplen, T.B., 2001, Distribution of oxygen-18 and deuterium in river waters across the United States: Hydrological Processes, v. 15, no. 7, p. 1363-1393.

Kernodle, J.M., 1996, Hydrogeology and steady-state simulation of ground-water flow in the San Juan Basin, New Mexico, Colorado, Arizona, and Utah: U.S. Geological Survey Water-Resources Investigations Report 95-4187, $117 \mathrm{p}$.

Kigoshi, K., 1971, Alpha-recoil thorium-234-Dissolution into water and the uranium-234/uranium-238 disequilibrium in nature: Science, v. 173, no. 3991, p. 47-48.

Kotler, J.M., Quinn, R.C., Foing, B.H., Martins, Z., and Ehrenfreund, P., 2011, Analysis of mineral matrices of planetary soil analogues from the Utah Desert: International Journal of Astrobiology, v. 10, no. 03, p. 221-229.

Kronfeld, J., and Adams, J.A.S., 1974, Hydrologic investigations of the groundwaters of central Texas using U-234/U-238 disequilibrium: Journal of Hydrology (Amsterdam), v. 22, no. 1-2, p. 77-78.

Krumholz, Lee R., McKinley, James P. Ulrich, Glenn A., Suflita, Joseph M., 1997, Confined subsurface microbial communities in Cretaceous rock: Nature (London), v. 386, no. 6620, p. 64-66.

Langmuir, Donald, 1978, Uranium solution-mineral equilibria at low temperatures with applications to sedimentary ore deposits: Geochimica et Cosmochimica Acta, v. 42, no. 6, part a, p. 547-569.

Laronne, J.B., 1977, Dissolution potential of surficial Mancos Shale and alluvium: Fort Collins, Colo., Colorado State University, $128 \mathrm{p}$.

Laronne, J.B., and Schumm, S.A., 1977, Evaluation of the storage of diffuse sources of salinity in the Upper Colorado River Basin: Fort Collins, Colo., Colorado State University, 84 p.

Leopold, L.B., Wolman, M.G., and Miller, J.P., 1964, Fluvial processes in geomorphology: San Francisco, Calif., W.H. Freeman and Company p. 548.

Levings, G.W., Kernodle, J.M., and Thorn, C.R., 1996, Summary of the San Juan structural basin regional aquifersystem analysis, New Mexico, Colorado, Arizona, and Utah: U.S. Geological Survey Water-Resources Investigations Report 95-4188, 55 p.
Leythaeuser, Detlev, 1973, Effects of weathering on organic matter in shales: Geochimica et Cosmochimica Acta, v. 37, no. 1, p. 113-120.

Lowe, Mike, and Wallace, Janae, 2001, Evaluation of potential geologic sources of nitrate contamination in ground water, Cedar Valley, Iron County, Utah, with emphasis on the Enoch area: Utah Geologic Survey, 50 p.

Lucas, L.L., and Unterweger, M.P., 2000, Comprehensive review and critical evaluation of the half-life of tritium: Journal of Research of the National Institute of Standards and Technology, v. 105, no. 4, p. 541-549.

Mast, A.M., Mills, T.J., Paschke, S.S., Keith, G., and Lindard, J.I., 2014, Mobilization of selenium from the Mancos Shale and associated soils in the Lower Uncompahgre River Basin, Colorado: Applied Geochemistry, v. 48, p. 16-27.

Mayer, B., 2005, Assessing sources and transformations of sulfate and nitrate in the hydrosphere using isotope techniques, in Aggarwal, P.K., ed., Isotopes in the water cycle-Past, present and future of a developing science: Dordrecht, Netherlands, Springer, p. 67-89.

McMahon, P.B., Bohlke, J.K., and Bruce, B.W., 1999, Denitrification in marine shales in northeastern Colorado: Water Resources Research, v. 35, no. 5, p. 1629-1642.

Morrison, S.J., Goodknight, C.S., Tigar, A.D., Bush, R.P., and Gil, A., 2012, Naturally occurring contamination in the Mancos Shale: Environmental Science \& Technology, v. 46, no. 3, p. 1379-1387.

Murphy, E.M., Ginn, T.R., and Phillips, J.L., 1996, Geochemical estimates of paleorecharge in the Pasco Basin: Evaluation of the chloride mass balance technique: Water Resources Research, v. 32, no. 9, p. 2853-2868.

National Atmospheric Deposition Program, 2014, National trends network: Accessed on June 30, 2014, at http://nadp. sws.uiuc.edu/NTN/.

National Ocean Sciences Accelerator Mass Spectrometry Facility, 2010, General statement of 14C procedures at the National Ocean Sciences AMS Facility, accessed July 2015 at http://www.whoi.edu/nosams/general-statement-of-14cprocedures.

Natural Resources Conservation Service, 2015, Web soil survey: Accessed on June 16, 2015, at http://websoilsurvey. nrcs.usda.gov/app/.

New Mexico Bureau of Geology and Mineral Resources, 2003, Geologic map of New Mexico: New Mexico Bureau of Geology and Mineral Resources, scale 1:500,000.

New Mexico Oil Conservation Division, 2015, OCD imaging: Accessed on June 16, 2015, at http://ocdimage.emnrd.state. nm.us/imaging/. 
Parker, C.G., 1963, Piping, a geomorphic agent in landform development of the drylands, in Land erosion, precipitations, hydrometry, soil moisture: International Association of Scientific Hydrologists Publication, v. 65, p. 103-113.

Phillips, F.M., Hogan, J.F., Mills, S.K., and Hendrickx, J.M.H., 2003, Environmental tracers applied to quantifying causes of salinity in arid-region rivers - Preliminary results from the Rio Grande, southwestern USA, in Alsharhan, A.S., and Wood, W.W., eds., Water resources perspectivesEvaluation, management, and policy: Amsterdam, Netherlands, Elsevier Science, p. 327-334.

Phillips, F.M., Peeters, L.A., and Tansey, M.K., 1986, Paleoclimatic inferences from an isotopic investigation of groundwater in the central San Juan Basin, New Mexico: Quaternary Research, v. 26, no. 2, p. 179-193.

Phillips, F.M., Tansey, M.K., and Peeters, L.A., 1989, An isotopic investigation of groundwater in the Central San Juan Basin, New Mexico-Carbon 14 dating as a basis for numerical flow modeling: Water Resources Research, v. 25, p. 2259-2273.

Pliler, R., and Adams, J.A.S., 1962, The distribution of thorium, uranium, and potassium in the Mancos Shale: Geochimica et Cosmochimica Acta, v. 26, p. 1115-1135.

Plummer, L.N., Bexfield, L.M., Anderholm, S.K., Sanford, W.E., and Busenberg, Eurybiades, 2004, Geochemical characterization of ground-water flow in the Santa Fe Group aquifer system, middle Rio Grande Basin, New Mexico: U.S. Geological Survey Water-Resources Investigations Report 03-4131, 395 p.

Plummer, L.N., and Friedman, L.C., 1999, Tracing and dating young ground water: U.S. Geological Survey Fact Sheet 134-99, 4 p.

Power, J.F., Bond, J.J., Sandoval, F.M., and Willis, W.O., 1974, Nitrification in Paleocene shale: Science, v. 183, no. 4129, p. 1077-1079.

Presser, T.S., 1994, The Kesterson effect: Environmental Management, v. 16, no. 3, p. 437-454.

Public Health Service, 1962, Stream surveys in vicinity of uranium mills-IV, area of Shiprock, New Mexico, November 1960: U.S. Department of Health, Education and Welfare, $23 \mathrm{p}$.

Quinn, J.E., 1957, Kerr-McGee Oil Industries, Inc. Shiprock uranium concentrator: Denver Equipment Co. Bulletin No. M4-B90, 12 p.

Ries, K.S., 1982, Stable oxygen and sulfur isotopes applied to tracing seepage from mine tailings: University of Arizona, Tucson, Master's thesis, $162 \mathrm{p}$.
Robertson, A.J., Henry, D.W., and Langman, J.B., 2013, Geochemical evidence of groundwater flow paths and the fate and transport of constituents of concern in the alluvial aquifer at Fort Wingate Depot Activity, New Mexico, 2009: U.S. Geological Survey Scientific Investigations Report 2013-5098, 89 p.

Sherman, H.M., Gierke, J.S., and Anderson, C.P., 2007, Controls on spatial variability of uranium in sandstone aquifers: Ground Water Monitoring \& Remediation, v. 27, no. 2, p. 106-118.

Smith, L.N., 1992, Quaternary history and landscape development of some tributary drainage basins north of the Chaco River, in Lucas, S.G., Kues, B.S., Williamson, T.E., and Hunt, A.P., eds., San Juan Basin IV: New Mexico Geological Society, Forty-Third Annual Field Conference, Socorro, N. Mex., p. 391-398.

Sterling, J.M., 2000, Spatial distribution of chloride and 36Cl deposition in the conterminous United States: Socorro, N. Mex., New Mexico Institute of Mining and Technology, Master's Thesis, $155 \mathrm{p}$.

Stone, W.J., 1986, Natural recharge in southwestern landscapes - Examples form New Mexico in Proceedings of the Conference on Southwest Ground Water Issues, Tempe, Arizona: Dublin, Ohio, National Water Well Association, p. 595-602.

Szabo, Zoltan, and Zapecza, O.S., 1991, Geologic and geochemical factors controlling uranium, radium-226, and radon-222 in groundwater, Newark Basin, New Jersey in Gunderson, L.C.S., and Wanty, R.B., eds., Field studies of radon rocks, soils, and water: U.S. Geological Survey Bulletin 1971, p. 243-265.

The Reston Groundwater Dating Laboratory, 2014, Chlorofluorocarbon analysis: U.S. Geological Survey, accessed on various dates, at http://water.usgs.gov/lab/ chlorofluorocarbons/.

The Reston Groundwater Dating Laboratory, 2015a, Analytical Procedures for Dissolved Gasses N2 / Ar: U.S. Geological Survey, accessed on various dates, at http:/water.usgs.gov/ lab/dissolved-gas/lab/analytical_procedures/.

The Reston Groundwater Dating Laboratory, 2015b, Analytical Procedures for Dissolved Gasses N2 / Ar: U.S. Geological Survey, accessed on various dates, at http:// water.usgs.gov/lab/software/USGS_CFC/.

The Sulfur Institute, 2014, Learn more about sulfur: Accessed on September 02, 2014, at http://www.sulphurinstitute.org/ learnmore/faq.cfm. 
Thomas, C.L., Wilson, R.M., Lusk, J.D., Bristol, R.S., and Shineman, A.R., 1998, Detailed study of selenium and selected constituents in water, bottom sediment, soil, and biota associated with irrigation drainage in the San Juan River area, New Mexico, 1991-95: U.S. Geological Survey Water-Resources Investigations Report 98-4213, 96 p.

Thomas, J.C., Leib, K.J., and Mayo, J.W., 2008, Analysis of dissolved selenium loading for selected sites in the Lower Gunnison River Basin, Colorado, 1978-2005: U.S. Geological Survey Scientific Investigations Report 20095048, 19 p.

Tricca, A., Porcelli, D., and Wasserburg, G.J., 2000, Factors controlling the groundwater transport of $\mathrm{U}, \mathrm{Th}, \mathrm{Ra}$, and Rn: Journal of Earth System Science, v. 109, no. 1, p. 95-108.

Tsosie, B.B., 1997, Hydrogeologic characterization of the floodplain that lies below the uranium mill Tailings Remedial Action Site at Shiprock, New Mexico: Socorro, N. Mex., New Mexico Institute of Mining and Technology, Master's Thesis, 75 p.

Tuttle, M.L.W., Fahy, Juli, Grauch, R.I., Ball, B.A., Chong, G.W., Elliott, J.G., Kosovich, J.J., Livo, K.E., and Stillings, L.L., 2007, Results of chemical analyses of soil, shale, and soil/shale extract from the Mancos Shale Formation in the Gunnison Gorge National Conservation Area, southwestern Colorado, and at Hanksville, Utah: U.S. Geological Survey Open-File Report 2007-1002D, 24 p.

Tuttle, M.L.W., Fahy, Juli, Grauch, R.I., Stillings, Lisa, Anonymous, 2009, Salt and selenium in the upper Colorado River-Processes of supply and transport from the Cretaceous Mancos Shale: Geological Society of America Abstracts with Programs, v. 41, no. 7, p. 682-682.

Tuttle, M.L.W., Fahy, J.W., Elliott, J.G., Grauch, R.I., and Stillings, L.L., 2014, Contaminants from Cretaceous black shale_-Natural weathering processes controlling contaminant cycling in Mancos Shale, Southwestern United States, with emphasis on salinity and selenium: Applied Geochemisty, v. 46, p. 57-71.

Tuttle, M.L.W, and Grauch, R.L., 2009, Salinization of the upper Colorado River-Fingerprinting geologic salt sources: U.S. Geological Survey Scientific Investigations Report 2009-5072, 62 p.

U.S. Department of Energy, 1996, Geophysical surveys at the UMTRA project Shiprock, New Mexico, site: U.S. Department of Energy, $20 \mathrm{p}$.

U.S. Department of Energy, 2000, Final site observational work plan for the Shiprock, New Mexico, UMTRA project site: U.S. Department of Energy, 497 p.
U.S. Department of Energy, 2011a, Geology and groundwater investigation Many Devils Wash, Shiprock site, New Mexico: U.S. Department of Energy, 40 p.

U.S. Department of Energy, 2011b, Natural contamination from the Mancos Shale: U.S. Department of Energy, 78 p.

U.S. Department of Energy, 2011c, Shiprock, New Mexico, Disposal Site: Grand Junction, Colo., U.S. Department of Energy Fact Sheet, 4 p.

U.S. Department of Energy, 2012a, Application of environmental isotopes to the evaluation of the origin of contamination in a desert arroyo-Many Devils Wash, Shiprock, N. Mex.: U.S. Department of Energy, 32 p.

U.S. Department of Energy, 2012b, Multivariate statistical analysis of water chemistry in evaluating the origin of contamination in Many Devils Wash, Shiprock, N. Mex.: U.S. Department of Energy, 62 p.

U.S. Department of Energy, 2012c, Shiprock, New Mexico, disposal cell internal water balance and cell conditions: U.S. Department of Energy, 24 p.

U.S. Department of Energy, 2014, Geospatial environmental mapping system: accessed on various dates at http://gems. $1 \mathrm{~m}$.doe.gov/\#\&site=SHP.

U.S. Department of the Interior, 2001, National Irrigation Water Quality Program Fact Sheet: Denver, Colo, 9 p.

U.S. Environmental Protection Agency, 2009, National Primary Drinking Water Regulations: EPA 816-F-09-004, $6 \mathrm{p}$.

U.S. Geological Survey, variously dated, National field manual for the collection of water-quality data: U.S. Geological Survey Techniques of Water-Resources Investigations, book 9, chaps. A1-A9. [Also available at http://water.usgs. gov/owq/FieldManual/.]

Wagenet, R.J., and Jurinak, J.J., 1978, Spatial variability of soluble salt content in a Mancos Shale watershed: Soil Science, v. 126, no. 6, p. 342.

Wang, Yang, Huntington, T.G., Osher, L.J., Wassenaar, L.I., Trumbore, S.E., Amundson, R.G., Harden, J.W., McKnight, D.M., Schiff, S.L., Aiken, G.R., Lyons, W. Berry, Aravena, Ramon O., Baron, J.S., 1998, Carbon cycling in terrestrial environments, in Kendall, C., and McDonnell, J.J., eds., Isotope tracers in catchment hydrology: Amsterdam, Elsevier Science B.V., p. 577-610.

Ward, A.W., 1990, Geologic map emphasizing the surficial deposits of the Farmington $30^{\prime}$ x 60' quadrangle, New Mexico and Colorado: U.S. Geological Survey Investigations Series Map I-1978, scale 1:100,000. 
Welch, A.H., and Preissler, A.M., 1986, Aqueous geochemistry of the Bradys Hot Spring geothermal area, Churchill County, Nevada in Subitzky, S., ed., Selected Papers in the Hydrologic Sciences: U.S. Geological Survey Water Supply Paper 2290, 36 p.

Western Regional Climate Center, 2012, Shiprock, New Mexico, period of record monthly climate summary: Accessed on November 14, 2012, at http://www.wrcc.dri. edu/cgi-bin/cliMAIN.pl?nm8284.

Whittig, L.D., Deyo, A.E., and Tanji, K.K., 1982, Evaporite mineral species in Mancos Shale and salt efflorescence, Upper Colorado River Basin: Soil Science Society of America Journal, v. 46, no. 3, p. 645-651.

Wigley, T.M.L., Plummer, L.N., and Pearson, F.J., Jr., 1978, Mass transfer and carbon isotope evolution in natural water systems: Geochimica et Cosmochimica Acta, v. 42, p. 22.
Wood, W.W., and Sanford, W.E., 1995, Chemical and isotopic methods for quantifying ground-water recharge in a regional, semiarid environment: Ground Water, v. 33, no. 3, p. $458-468$.

Wright, A.F., 1979, Bibliography of the geology and hydrology, San Juan Basin, New Mexico, Colorado, Arizona, and Utah: U.S. Geological Survey Bulletin 1481, p. 123.

Wright, W.G., 1999, Oxidation and mobilization of selenium by nitrate in irrigation drainage: Journal of Environmental Quality, v. 28, no. 4, p. 1182-1187.

Zielinski, R.A., Chafin, D.T., Banta, E.R., and Szabo, B.J., 1997, Use of $234 \mathrm{U}$ and $238 \mathrm{U}$ isotopes to evaluate contamination of near-surface groundwater with uraniummill effluent-A case study in south-central Colorado, U.S.A.: Berlin, Environmental Geology, v. 32, no. 2, p. 124-136. 
